\title{
Métodos de pontos interiores aplicados ao problema de regressão pela norma $L_{p}$
}

\author{
Daniela Renata Cantane
}

Orientador: Prof. Dr. Aurelio Ribeiro Leite de Oliveira

Dissertação apresentada ao Instituto de Ciências Matemáticas e de Computação - ICMC-USP, como parte dos requisitos para obtenção do título de Mestre em Ciências - Área: Ciências da Computação e Matemática Computacional.

USP - São Carlos - SP

Setembro/2004 
À minha família. 


\section{Agradecimentos}

À Deus, por estar sempre abençoando minha vida e iluminando meus caminhos durante toda essa caminhada.

Aos meus pais, Cidinha e Carlinhos e aos meus irmãos, Daniel e Diego, pelo apoio e incentivo aos meus estudos. Agradeço por estarem sempre presentes em minha vida.

Ao meu namorado, Daniel, pela compreensão nos momentos que estive ausente, pelo seu amor, carinho e amizade durante todos estes anos.

Ao meu orientador, pela paciência e dedicação ao longo do desenvolvimento do projeto e por ter me concedido esta oportunidade.

Aos professores e funcionários da USP que contribuiram para a minha formação de uma forma em geral.

Às minhas amigas "irmãzinhas", Aline, Lilian, Kelly, Cecília, Glaucia e Sônia que sempre estiveram dispostas a ajudar quando necessitei.

À FAPESP - Fundação de Amparo e Apoio à Pesquisa do Estado de São Paulo, pelo apoio financeiro. 


\section{Resumo}

Neste trabalho a família de métodos de pontos interiores barreira logarítmica é desenvolvida para o problema de regressão pela norma $L_{p}$ e a estrutura matricial resultante é explorada objetivando uma implementação eficiente. Apresentamos alguns conceitos sobre métodos de pontos interiores necessários para o desenvolvimento do método e descrevemos um método de convergência quadrática previamente conhecido. Uma implementação em Matlab dos métodos de pontos interiores desenvolvidos é comparada com uma implementação do método quadrático existente, obtendo desempenho computacional superior.

\section{Abstract}

In this work the family of logarithmic barrier interior point methods is developed for the norm $L_{p}$ fitting problem and the resultant matrix structure is exploited in order to have an efficient implementation. We introduce some concepts about interior point methods necessary for the development of the method and describe a previously known quadratic convergent problem. An implementation in Matlab of the interior point methods developed is compared with an implementation of the known quadratic method obtaining better computational performance. 


\section{Conteúdo}

Resumo iv

Abstract $\quad$ iv

1 Introdução 1

2 Métodos de Pontos Interiores 4

2.1 Conceitos Iniciais sobre Pontos Interiores . . . . . . . . . . . . . . . . 4

2.1 .1 Otimização Linear . . . . . . . . . . . . . . . . . . . . . . . . . 4

2.1 .2 Otimização Não Linear . . . . . . . . . . . . . . . . . . . . . 6

2.1.3 Convexidade....................... 8

2.2 Método de Newton . . . . . . . . . . . . . . . . . . . . . 8

2.2.1 Método de Newton para uma variável . . . . . . . . . . . . . . . 9

2.2.2 Método de Newton para várias variáveis . . . . . . . . . . . 9

2.3 Método de Pontos Interiores Primal-Dual . . . . . . . . . . . . . . . . . . 10

2.3.1 Método Primal-Dual Afim-Escala . . . . . . . . . . . . . . . . . . . 10

2.3.2 Método Primal-Dual Clássico . . . . . . . . . . . . . . . . . 14

2.4 Método de Pontos Interiores Barreira Logarítmica . . . . . . . . . . . . . 16

2.4.1 Critério de Convergência . . . . . . . . . . . . . . . . . . . 19

2.4 .2 Inicialização . . . . . . . . . . . . . . . . . . . . . . 19

2.5 Método de Pontos Interiores Barreira Logarítmica Preditor-Corretor . . . . 20

3 O Problema de Regressão $L_{p}$

3.1 O Problema de Regressão pela Norma $L_{p} \ldots \ldots$. . . . . . . . . . . . . . . . 24 
3.2 Métodos Pré-Existentes . . . . . . . . . . . . . . . . . 26

3.2.1 Métodos de Relaxação por Coluna para o problema de norma mínima 26

3.2 .2 Método GNCS . . . . . . . . . . . . . . . . . . 27

4 Métodos de Pontos Interiores Aplicados ao Problema de Regressão pela $\begin{array}{ll}\text { Norma } L_{p} & 38\end{array}$

4.1 Método Barreira Logarítmica . . . . . . . . . . . . . . . . . . 38

4.1 .1 Critério de Convergência . . . . . . . . . . . . . . . . . . 44

4.1 .2 Pontos Iniciais . . . . . . . . . . . . . . . . . . . . . 44

4.1.3 Algumas Considerações . . . . . . . . . . . . . . . . . . . . . . . 45

4.2 Método Preditor-Corretor . . . . . . . . . . . . . . . . 45

4.2.1 Algumas Considerações . . . . . . . . . . . . . . . . . . . . . 50

4.3 Método Primal-Dual Barreira Logarítmica . . . . . . . . . . . . . . . 50

4.3.1 Critério de Convergência . . . . . . . . . . . . . . 56

4.3.2 Algumas Considerações . . . . . . . . . . . . . . . . . 57

4.4 Método Primal-Dual Preditor-Corretor . . . . . . . . . . . . . 57

4.4.1 Algumas Considerações . . . . . . . . . . . . . . . . . . . . 63

4.5 Regressão Polinomial . . . . . . . . . . . . . . . . . . . . 63

$\begin{array}{lll}5 & \text { Resultados Computacionais } & 68\end{array}$

6 Conclusões e Perspectivas Futuras $\quad 97$

6.1 Conclusões . . . . . . . . . . . . . . . . . . . . . . . . . 97

6.2 Perspectivas Futuras . . . . . . . . . . . . . . . . . . . . 98 


\section{Lista de Tabelas}

5.1 Resultados computacionais utilizando a função $f_{1}(z)$. . . . . . . . . . 71

5.2 Resultados computacionais utilizando a função $f_{2}(z) \ldots$. . . . . . . . . 71

5.3 Utilizando a função $f_{1}(z): z_{u}=u, z_{v}=v \ldots \ldots \ldots$. . . . . . . 73

5.4 Utilizando a função $f_{2}(z): z_{u}=u, z_{v}=v \ldots \ldots \ldots \ldots$. . . . . . . . .

5.5 Utilizando a função $f_{1}(z)$ e $z_{u}=z_{v}=e \ldots \ldots \ldots \ldots$. . . . . . 74

5.6 Utilizando a função $f_{2}(z)$ e $z_{u}=z_{v}=e \ldots \ldots \ldots \ldots$. . . . . . 74

5.7 Utilizando a função $f_{1}(z)$ e $z_{u}=((\lambda+1) / 2)$ e e $z_{v}=((\lambda-1) / 2) e . \ldots .75$

5.8 Utilizando a função $f_{2}(z)$ e $z_{u}=((\lambda+1) / 2)$ e e $z_{v}=((\lambda-1) / 2)$ e. . . . . 75

5.9 Resultados computacionais utilizando a função $\sin x$. . . . . . . . . . 85

5.10 Resultados computacionais utilizando a função $\sin x$. . . . . . . . . . . . 85

5.11 Resultados computacionais utilizando a função $\sin x$. . . . . . . . . . 86

5.12 Resultados computacionais utilizando a função $\sin x$. . . . . . . . . . 86

5.13 Resultados computacionais utilizando a função $\sinh x \ldots$. . . . . . . . . . 87

5.14 Resultados computacionais utilizando a função $\sinh x$. . . . . . . . . . . . 87

5.15 Resultados computacionais utilizando a função $\sinh x \ldots$. . . . . . . . 88

5.16 Resultados computacionais utilizando a função $\sinh x \ldots$. . . . . . . . . 88

5.17 Resultados computacionais utilizando a função $\ln x$. . . . . . . . . . . . 89

5.18 Resultados computacionais utilizando a função $\ln x$. . . . . . . . . . . . 89

5.19 Resultados computacionais utilizando a função $\ln x$. . . . . . . . . . . . . 90

5.20 Resultados computacionais utilizando a função $\ln x$. . . . . . . . . . . . . 90

5.21 Resultados computacionais utilizando a função $\exp x$. . . . . . . . . . . 91

5.22 Resultados computacionais utilizando a função $\exp x$. . . . . . . . . . . 91

5.23 Resultados computacionais utilizando a função $\exp x$. . . . . . . . . . . . 92 
5.24 Resultados computacionais utilizando a função $\exp x$. . . . . . . . . . . . 92

5.25 Resultados computacionais utilizando a função $\exp x^{2} \ldots$. . . . . . . . . . 93

5.26 Resultados computacionais utilizando a função $\exp x^{2} \ldots$. . . . . . . . . . 93

5.27 Resultados computacionais utilizando a função $\exp x^{2} \ldots$. . . . . . . . . 94

5.28 Resultados computacionais utilizando a função $\exp x^{2} \ldots$. . . . . . . . . . 94

5.29 Resultados computacionais utilizando o problema de grande porte. . . . . . 95

5.30 Resultados computacionais utilizando o problema de grande porte. . . . . . 95

5.31 Resultados computacionais utilizando o problema de grande porte. . . . . . 96 


\section{Capítulo 1}

\section{Introdução}

Desde o surgimento dos métodos de pontos interiores para otimização linear, códigos computacionais baseados nessas idéias vem se apresentando como alternativas eficientes para solução de problemas de grande porte [1, 10, 15, 19].

Uma linha de pesquisa importante nesta área considera classes específicas de problemas e explora as particularidades da estrutura matricial com o objetivo de obter implementações ainda mais eficientes, inclusive para problemas com restrições lineares e função objetivo não linear $[5,20,21,22,23,24,25]$.

O objetivo deste trabalho consiste no desenvolvimento dos métodos de pontos interiores para o problema de regressão pela norma $L_{p}, 1<p<2$, no estudo da estrutura matricial resultante e na implementação eficiente do método desenvolvido. Os resultados obtidos serão comparados com uma implementação do método proposto em [13].

Dada uma classe de problemas, a forma padrão para o desenvolvimento de um método de pontos interiores consiste na aplicação do método de Newton às condições de otimalidade desconsiderando as restrições de capacidade. O método resultante é essencialmente um método de pontos interiores específico para esta classe de problemas. Algumas alterações, através da introdução de perturbações, são necessárias para obter um método eficiente $[29,30]$. De forma análoga, desenvolve-se uma das variantes mais importantes 
dos métodos de pontos interiores, o método preditor-corretor [16].

A etapa seguinte desta abordagem consiste na exploração eficiente da estrutura matricial do problema. É sempre importante lembrar que a resolução de um sistema linear, em geral simétrico, consiste no passo mais caro, em termos computacionais, de cada iteração dos métodos de pontos interiores. Desta forma, a exploração da estrutura matricial pode levar a métodos de pontos interiores mais eficientes que os métodos genéricos aplicados a um problema particular. As idéias desenvolvidas em [20, 21] para os problemas de regressão $L_{1}$ e $L_{\infty}$ também podem ser adaptadas a este problema devido à semelhança das estruturas matriciais com o problema de regressão $L_{p}$.

O problema de regressão

$$
\min _{x \in \mathbb{R}^{m}}\|A x-b\|_{p}^{p}
$$

onde $A=\left[a_{1}, \ldots, a_{n}\right] \in \mathbb{R}^{m \times n}, b \in \mathbb{R}^{n}$ e $n>m$, tem inúmeras aplicações em diversas áreas de ciências e engenharias. As normas mais utilizadas são as normas $L_{1}, L_{2}$ e $L_{\infty}$. A norma $L_{2}$ é muito popular entre outros motivos por permitir uma solução direta. Por sua vez a norma $L_{1}$ permite diminuir o efeito de pontos discrepantes enquanto que a norma $L_{\infty}$ garante proteção contra o pior caso.

O método IRLS iteratively reweighted least-squares [17] foi por muito tempo a única alternativa prática para a resolução deste problema para outros valores de $p$. Mais recentemente este método foi aperfeiçoado, no que diz respeito à robustez, através da inclusão de uma busca linear [13]. No mesmo trabalho, foi também proposto um novo método que apresenta características similares aos métodos de pontos interiores. Este método apresentou resultados computacionais superiores ao IRLS.

Ambos métodos apresentados em [13] têm uma importante desvantagem: a busca linear é computacionalmente cara. Isto nos motivou o estudo dos métodos de pontos interiores aplicados a este problema que obtém resultados computacionais superiores, repetindo o desempenho obtido na minimização pelas normas $L_{1}$ e $L_{\infty}$ em [20, 21], respectivamente. 
No Capítulo 2, descrevemos alguns conceitos sobre métodos de pontos interiores. Descrevemos também o método de Newton que será utilizado para o desenvolvimento dos métodos de pontos interiores barreira logarítmica, primal-dual e preditor-corretor.

No Capítulo 3, apresentamos o problema de regressão pela norma $L_{p}$ e o método de convergência quadrática GNCS, desenvolvido em [13], que será utilizado para compararmos com o método de pontos interiores desenvolvido no capítulo seguinte.

Desenvolvemos, no Capítulo 4, os métodos de pontos interiores barreira logarítmica, primal-dual e preditor-corretor nos quais são aplicados ao problema de regressão pela norma $L_{p}$ e as estruturas matriciais resultante são estudadas.

No Capítulo 5, apresentamos os resultados computacionais obtidos em nossos métodos e comparamos com os resultados do método existente apresentado no Capítulo 3.

No Capítulo 6, apresentamos as conclusões e perspectivas futuras do nosso trabalho e por último encontram-se as referências bibliográficas. 


\section{Capítulo 2}

\section{Métodos de Pontos Interiores}

Neste capítulo, descrevemos conceitos sobre pontos interiores, método de Newton, métodos barreira logarítmica, primal-dual e preditor-corretor que serão aplicados ao problema de regressão $L_{p}$.

\subsection{Conceitos Iniciais sobre Pontos Interiores}

Antes de descrevermos os métodos de pontos interiores, devemos caracterizar o que é um problema de otimização linear, um problema de otimização não linear e um ponto interior em problemas desse tipo.

Os problemas de otimização linear derivam da construção de uma representação matemática para um problema real em que se quer minimizar ou maximizar uma função objetivo linear, ao mesmo tempo em que as variáveis estão sujeitas a determinadas restrições também lineares. Para os problemas de otimização não linear, temos uma função objetivo não linear e \ou restrições não lineares. Um ponto interior é aquele em que todas as variáveis se encontram estritamente dentro de seus limites.

\subsubsection{Otimização Linear}

Seja um problema de otimização linear na forma padrão dado por 


$$
\begin{array}{cc}
\min & c^{t} x \\
\text { sa } & A x=b \\
& x \geq 0
\end{array}
$$

onde $A$ é uma matriz, $x$ é um vetor coluna de variáveis primais, $b$ e $c$ são vetores de constantes.

Esta formulação é denominada problema primal e está associada ao problema dual

$$
\begin{array}{cccc}
\max & b^{t} y \quad \Leftrightarrow \max & b^{t} y \\
\text { sa } & A^{t} y \leq c & \text { sa } & A^{t} y+z=c \\
& y \text { livre } & & z \geq 0, \quad y \text { livre }
\end{array}
$$

onde y é um vetor coluna de variáveis duais e z é a variável de folga complementar.

Na forma padrão, $x>0$ é um ponto interior no problema primal e $z>0$ é um ponto interior para o problema dual. No problema primal $A x=b ; x>0$ é um ponto interior factível e no problema dual $A^{t} y+z=c ; z>0$ é um ponto interior factível.

Um outro conceito que será utilizado para a construção de métodos de pontos interiores é o gap. O gap é a diferença entre os valores das funções objetivo para o primal e o dual de um mesmo problema, ou seja, $\gamma=c^{t} x-b^{t} y$ [30]. É possível mostrar que para um ponto primal e dual factível, o gap é dado por $\gamma=c^{t} x-b^{t} y=z^{t} x$.

Por último, é possível determinar as condições de otimalidade. Um dado ponto $(x, y, z)$ é ótimo para os problemas primal e dual se e somente se as seguintes condições são satisfeitas [2]:

(i) Primal factível: $A x=b, x \geq 0$;

(ii) Dual factível: $A^{t} y+z=c, z \geq 0$;

(iii) Complementaridade: $X Z e=0$, ou seja, $x_{i} z_{i}=0, \quad i=1, \ldots, n$. onde $e$ é o vetor coluna de dimensão $n$ com todos os elementos iguais a 1 , as 
notações $X=\operatorname{diag}(x)$ e $Z=\operatorname{diag}(z)$ são utilizadas e $X Z e=0$ equivale à $x_{i} z_{i}=0, i=1, \ldots, n$.

\subsubsection{Otimização Não Linear}

Agora, veremos a formulação de um problema de otimização não linear [3]. Em geral, um problema de otimização não linear é da forma:

$$
\begin{aligned}
& \min f(x) \\
& \text { sa } h_{1}(x)=0 \\
& h_{m}(x)=0 \\
& g_{1}(x) \leq 0 \\
& g_{p}(x) \leq 0 \\
& x \in \Omega \text {, }
\end{aligned}
$$

onde as funções $f, h_{i}$ e $g_{i}$ são contínuas e normalmente $\in \mathbb{C}^{2}$ e $\Omega \subset \mathbb{R}^{n}$.

Devido ao fato de que em nosso trabalho utilizaremos problema com restrição de igualdade, serão abordadas somente as condições de otimalidade relativa ao mesmo.

Seja o problema

$$
\begin{array}{cc}
\min & f(x) \\
\text { sa } & h(x)=0
\end{array}
$$

onde $h(x)$ é um vetor de restrições de igualdade.

A função Lagrangiana associada ao problema é dada por

$$
L(x, y)=f(x)+y^{t} h(x)
$$

A função Lagrangiana torna o problema irrestrito.

As condições de otimalidade para o problema (2.3) são dadas por: 
(i) Condições necessárias de 1å ordem:

Derivando a função Lagrangiana com relação a $x$ e a $y$, obtemos

$$
\nabla L(x, y)=\left\{\begin{array}{c}
\nabla f(x)+\sum_{i=1}^{m} y_{i} \nabla h_{i}(x)=0 \\
h_{i}(x)=0, \quad i=1, \ldots, m \\
x \in \mathbb{R}^{n} .
\end{array}\right.
$$

Este sistema fornece os candidatos a ponto ótimo.

(ii) Condição necessária de 2a ordem:

Um ponto $x^{*}$ satisfazendo as restrições $h(x)=0$ é chamado ponto regular (ponto candidato a ótimo) das restrições se os vetores gradientes $\nabla h_{1}\left(x^{*}\right), \nabla h_{2}\left(x^{*}\right), \ldots$, $\nabla h_{m}\left(x^{*}\right)$ forem linearmente independentes.

Um conjunto de restrições de igualdade $h(x)=0$ define um subconjunto do $\mathbb{R}^{n}$ chamado de hipersuperfície. Em um ponto regular $x^{*}$ da hipersuperfície definida por $h(x)=0$ o plano tangente é dado por:

$$
M=\left\{y \mid \nabla^{t} h\left(x^{*}\right) y=0\right\}
$$

onde

$$
\nabla h\left(x^{*}\right)=\left[\begin{array}{c}
\nabla h_{1}\left(x^{*}\right) \\
\vdots \\
\nabla h_{m}\left(x^{*}\right)
\end{array}\right] .
$$

Temos que $f$ e $h \in \mathbb{C}^{2}$. Seja $x^{*}$ um ponto regular das restrições $h(x)=0$ e um ponto extremo local (mínimo ou máximo) de $f(x)$ sujeito à restrição $h(x)=0$. Então existe um vetor $y^{t} \in \mathbb{R}^{n} \mid \nabla f\left(x^{*}\right)+y^{t} \nabla h\left(x^{*}\right)=0$, onde $y$ é o vetor multiplicador de Lagrange.

Considere $M=\left\{y \mid \nabla^{t} h\left(x^{*}\right) y=0\right\}$. Então a matriz $L\left(x^{*}\right)=F\left(x^{*}\right)+y^{t} H\left(x^{*}\right)$ é semidefinida positiva em $M$, isto é,

$$
y^{t} L\left(x^{*}\right) y \geq 0, \quad \forall y \in M,
$$

onde $L\left(x^{*}\right)=\nabla^{2} L\left(x^{*}\right), F\left(x^{*}\right)=\nabla f\left(x^{*}\right)$ e $H\left(x^{*}\right)=\nabla h\left(x^{*}\right)$. 
(iii) Condição suficiente de 2 a ordem:

Seja $x^{*}$ regular tal que $\nabla f\left(x^{*}\right)+y^{t} \nabla h\left(x^{*}\right)=0$. Suponha que $L\left(x^{*}\right)=F\left(x^{*}\right)+$ $y^{t} H\left(x^{*}\right)$ seja definida positiva em $M$, isto é,

$$
y^{t} L\left(x^{*}\right) y>0, \quad \forall y \in M
$$

Então $x^{*}$ é o ponto de mínimo local sujeito a $h_{i}(x)=0$.

\subsubsection{Convexidade}

Definição 2.1.1 (Conjuntos Convexos) Seja $\Omega \subset \mathbb{R}^{n}, \Omega$ é dito convexo se o segmento de reta que une quaisquer dois pontos de $\Omega$ está contido em $\Omega$, ou seja, $\forall x_{1}, x_{2} \in \Omega$, $\lambda x_{1}+(1-\lambda) x_{2} \in \Omega, \lambda \in[0,1]$.

Definição 2.1.2 (Funções Convexas) Seja $f: S \rightarrow E_{1}$, onde $S$ é um conjunto convexo não vazio em $E_{n}$. A função $f$ é dita convexa em $S$ se

$$
f\left(\lambda x_{1}+(1-\lambda) x_{2}\right) \leq \lambda f\left(x_{1}\right)+(1-\lambda) f\left(x_{2}\right)
$$

para todo $x_{1}, x_{2} \in S$ e $\lambda \in(0,1)$.

Se para todo $\lambda \in(0,1)$ e $x_{1} \neq x_{2}$ vale a desigualdade estrita então a função é denominada estritamente convexa.

Definição 2.1.3 (Funções Côncavas) A função $f: S \rightarrow E_{1}$ é denominada côncava (estritamente côncava) em $S$ se -f é convexa (estritamente convexa) em $S$.

\subsection{Método de Newton}

Os métodos de pontos interiores consistem na aplicação do método de Newton às condições de otimalidade desconsiderando as desigualdades e partindo de um ponto interior [30]. Para tanto, apresentaremos o método de Newton para uma variável e a seguir para várias variáveis. Para mais detalhes sobre o método de Newton e suas características ver $[3,14]$. 


\subsubsection{Método de Newton para uma variável}

Seja $x \in \Omega$ tal que $\phi(x)=0$. Para encontrarmos o valor de $x$ utilizamos aproximações sucessivas da função $\phi(x)$ em torno dos pontos $x^{0}, x^{1}, \ldots, x^{k}$, até que o ponto $x^{k}$ seja tal que $\phi\left(x^{k}\right) \approx 0$. Vamos utilizar a fórmula de Taylor em torno do ponto $x^{0}$ :

$$
0=\phi(x)=\phi\left(x^{0}\right)+\phi^{\prime}\left(x^{0}\right)\left(x-x^{0}\right)+\frac{\phi^{\prime \prime}\left(x^{0}\right)}{2 !}\left(x-x^{0}\right)^{2}+\ldots
$$

Aproximamos $\phi\left(x^{1}\right)$ até o termo linear da série, ou seja,

$$
0 \simeq \phi\left(x^{0}\right)+\phi^{\prime}\left(x^{0}\right)\left(x^{1}-x^{0}\right) \Rightarrow x^{1}=x^{0}-\frac{\phi\left(x^{0}\right)}{\phi^{\prime}\left(x^{0}\right)} .
$$

Podemos aplicar esta fórmula para obter $x^{1}$ e depois calcular o valor de $x^{k}$ sucessivamente, até que $\phi\left(x^{k}\right) \simeq 0$. Assim, construímos o método de Newton para uma variável:

Dado $x^{0}$

Para $k=0,1, \ldots$, faça

$$
\begin{aligned}
& d^{k}=-\frac{\phi\left(x^{k}\right)}{\phi^{\prime}\left(x^{k}\right)} \\
& x^{k+1}=x^{k}+d^{k}
\end{aligned}
$$

Até convergir (ou seja, $\phi\left(x^{k}\right) \simeq 0$ ).

\subsubsection{Método de Newton para várias variáveis}

Seja $x \in \mathbb{R}^{n}$ tal que $\phi_{i}(x)=0$, para $i=1,2, \ldots, n$. Novamente, para encontrarmos o valor de $x$ utilizamos aproximações sucessivas da função $\phi(x)$ em torno dos pontos $x^{0}, x^{1}, \ldots, x^{k}$, até que o ponto $x^{k}$ seja tal que $\phi_{i}\left(x^{k}\right) \simeq 0$. Aplicando a fórmula de Taylor para várias variáveis em torno de $x^{0}$ :

$$
0=\phi_{i}(x)=\phi_{i}\left(x^{0}\right)+\left[\nabla \phi_{i}\left(x^{0}\right)\right]^{t}\left(x-x^{0}\right)+\ldots, \quad \text { para } i=1, \ldots, n,
$$

onde

$$
\nabla \phi_{i}(x)=\left[\begin{array}{c}
\frac{\partial \phi_{i}(x)}{\partial x^{1}} \\
\vdots \\
\frac{\partial \phi_{i}(x)}{\partial x^{n}}
\end{array}\right]
$$


Aproximando $\phi_{i}\left(x^{1}\right)$ até o termo linear da série, obtemos

$$
0 \simeq \phi_{i}\left(x^{1}\right)=\phi_{i}\left(x^{0}\right)+\left[\nabla \phi_{i}\left(x^{0}\right)\right]^{t}\left(x^{1}-x^{0}\right), \quad \text { para } i=1, \ldots, n
$$

ou seja,

$$
\begin{gathered}
-\phi_{1}\left(x^{0}\right)=\left[\nabla \phi_{1}\left(x^{0}\right)\right]^{t}\left(x^{1}-x^{0}\right) \\
\vdots \\
-\phi_{n}\left(x^{0}\right)=\left[\nabla \phi_{n}\left(x^{0}\right)\right]^{t}\left(x^{1}-x^{0}\right) .
\end{gathered}
$$

Sejam $J\left(x^{0}\right)=\left[\begin{array}{c}{\left[\nabla \phi_{1}\left(x^{0}\right)\right]^{t}} \\ \vdots \\ {\left[\nabla \phi_{n}\left(x^{0}\right)\right]^{t}}\end{array}\right]$ a matriz Jacobiana e $F\left(x^{0}\right)=\left[\begin{array}{c}\phi_{1}\left(x^{0}\right) \\ \vdots \\ \phi_{n}\left(x^{0}\right)\end{array}\right]$. Então,

$$
-F\left(x^{0}\right)=J\left(x^{0}\right)\left(x^{1}-x^{0}\right) \Rightarrow x^{1}=x^{0}-\left[J\left(x^{0}\right)\right]^{-1} F\left(x^{0}\right) .
$$

Construímos, assim, o método de Newton para várias variáveis:

Dado $x^{0} \in \mathbb{R}^{n}$

Para $k=0,1, \ldots$, faça

$$
\begin{aligned}
& d^{k}=-\left[J\left(x^{k}\right)\right]^{-1} F\left(x^{k}\right) \\
& x^{k+1}=x^{k}+d^{k}
\end{aligned}
$$

Até convergir.

\subsection{Método de Pontos Interiores Primal-Dual}

Nesta seção, veremos os métodos de pontos interiores primal-dual afim-escala [18] e primal-dual clássico.

\subsubsection{Método Primal-Dual Afim-Escala}

Seja um problema com a formulação primal e dual igual a definida em (2.1) e (2.2) respectivamente. A idéia para se construir um método de pontos interiores consiste em 
aplicar o método de Newton ao sistema $F(x, y, z)=0$ formado pelas condições de otimalidade desconsiderando $(x, z) \geq 0$, resolvendo os problemas primal e dual simultaneamente. Sejam $x^{0}, y^{0}, z^{0},\left(x^{0}, z^{0}\right)>0$ um ponto interior. Temos que $F\left(x^{0}, y^{0}, z^{0}\right)$ é dado por

$$
F\left(x^{0}, y^{0}, z^{0}\right)=\left(\begin{array}{c}
A x^{0}-b \\
A^{t} y^{0}+z^{0}-c \\
X^{0} Z^{0} e
\end{array}\right)=-\left(\begin{array}{c}
r_{p}^{0} \\
r_{d}^{0} \\
r_{a}^{0}
\end{array}\right) .
$$

Utilizando o método de Newton para várias variáveis, obtemos

$$
\left(x^{1}, y^{1}, z^{1}\right)=\left(x^{0}, y^{0}, z^{0}\right)-\left[J\left(x^{0}, y^{0}, z^{0}\right)\right]^{-1} F\left(x^{0}, y^{0}, z^{0}\right)
$$

onde $J\left(x^{0}, y^{0}, z^{0}\right)=\left(\begin{array}{ccc}A & 0 & 0 \\ 0 & A^{t} & I \\ Z^{0} & 0 & X^{0}\end{array}\right)$.

Assim, $d^{0}$ será dado por

$$
d^{0}=\left(\begin{array}{ccc}
A & 0 & 0 \\
0 & A^{t} & I \\
Z^{0} & 0 & X^{0}
\end{array}\right)^{-1}\left(\begin{array}{c}
r_{p}^{0} \\
r_{d}^{0} \\
r_{a}^{0}
\end{array}\right)=\left(\begin{array}{c}
d x^{0} \\
d y^{0} \\
d z^{0}
\end{array}\right)
$$

Reescrevendo o sistema acima e por facilidade de notação ignorando o índice 0 temos,

$$
\left(\begin{array}{ccc}
A & 0 & 0 \\
0 & A^{t} & I \\
Z & 0 & X
\end{array}\right)\left(\begin{array}{c}
d x \\
d y \\
d z
\end{array}\right)=\left(\begin{array}{c}
r_{p} \\
r_{d} \\
r_{a}
\end{array}\right)
$$

Resolvendo o sistema (2.4), obtemos as direções $d x, d y$ e $d z$. Assim, temos o sistema

$$
\begin{aligned}
A d x & =r_{p} \\
A^{t} d y+d z & =r_{d} \\
Z d x+X d z & =r_{a}
\end{aligned}
$$

Da Equação (2.7), obtemos

$$
d z=X^{-1}\left(r_{a}-Z d x\right)
$$


Agora, da Equação (2.6), obtemos

$$
\begin{gathered}
A^{t} d y+X^{-1}\left(r_{a}-Z d x\right)=r_{d} \\
\Rightarrow A^{t} d y-X^{-1} Z d x=r_{d}-X^{-1} r_{a} .
\end{gathered}
$$

Definindo $D=X^{-1} Z$, obtemos

$$
\begin{gathered}
A^{t} d y-D d x=r_{d}-X^{-1} r_{a} \\
\Rightarrow d x=D^{-1}\left(A^{t} d y-r_{d}+X^{-1} r_{a}\right) .
\end{gathered}
$$

Substituindo a última equação em (2.5), temos

$$
\begin{gathered}
A D^{-1}\left(A^{t} d y-r_{d}+X^{-1} r_{a}\right)=r_{p} \\
\Rightarrow\left(A D^{-1} A^{t}\right) d y=r_{p}+A D^{-1} r_{d}-A D^{-1} X^{-1} r_{a} \\
\Rightarrow d y=\left(A D^{-1} A^{t}\right)^{-1}\left(r_{p}+A D^{-1} r_{d}-A D^{-1} X^{-1} r_{a}\right) .
\end{gathered}
$$

Temos que $A D^{-1} A^{t}$ tem dimensão $m, \operatorname{posto}(A)=m$, é simétrica e definida positiva. Podemos escrever

$$
A D^{-1} A^{t}=L L^{t}
$$

ou seja, podemos calcular a decomposição de Cholesky de $A D^{-1} A^{t}$. A ordem de escolha dos pivôs da diagonal não altera a estabilidade numérica.

A estrutura esparsa de $A D^{-1} A^{t}$ não varia com as iterações. Portanto, podemos utilizar a mesma sequência de pivôs obtidas por alguma eurístia de reordenamento [] em todas as iterações reduzindo o enchimento (elementos não nulos em L que são nulos em $\left.A D^{-1} A^{t}\right)$ da matriz na decomposição de Cholesky. A matriz $A D^{-1} A^{t}$ é permutada uma única vez antes de iniciar as iterações.

Podemos resumir estes cálculos como se segue.

\section{Método primal-dual afim-escala [18]}


Dados $\left(x^{0}, y^{0}, z^{0}\right)$ interior e $\tau \in(0,1)$

Para $k=0,1, \ldots$, faça

$$
\begin{aligned}
r_{p}^{k} & =b-A x^{k} \\
r_{d}^{k} & =c-A^{t} y^{k}-z^{k} \\
r_{a}^{k} & =-X^{k} Z^{k} e \\
d y^{k} & =\left[A\left(D^{k}\right)^{-1} A^{t}\right]^{-1}\left[r_{p}^{k}+A\left(D^{k}\right)^{-1} r_{d}^{k}-A\left(D^{k}\right)^{-1}\left(X^{k}\right)^{-1} r_{a}^{k}\right] \\
d x^{k} & =\left(D^{k}\right)^{-1}\left[A^{t} d y^{k}-r_{d}^{k}+\left(X^{k}\right)^{-1} r_{a}^{k}\right] \\
d z^{k} & =\left(X^{k}\right)^{-1}\left[r_{a}^{k}-Z^{k} d x^{k}\right] \\
\rho_{p} & =\min _{d x_{i}<0}\left\{-\frac{x_{i}}{d x_{i}}\right\} \\
\rho_{d} & =\min _{d z_{i}<0}\left\{-\frac{z_{i}}{d z_{i}}\right\} \\
\alpha_{p}^{k} & =\min \left\{1, \tau \rho_{p}\right\} \\
\alpha_{d}^{k} & =\min \left\{1, \tau \rho_{d}\right\} \\
x^{k+1} & =x^{k}+\alpha_{p}^{k} d x^{k} \quad\left(\alpha_{p}^{k} \text { é tal que } x^{k+1}>0\right) \\
y^{k+1} & =y^{k}+\alpha_{d}^{k} d y^{k} \\
z^{k+1} & =z^{k}+\alpha_{d}^{k} d z^{k} \quad\left(\alpha_{d}^{k} \text { é tal que } z^{k+1}>0\right)
\end{aligned}
$$

Até convergir.

Observação 2.3.1 Dados $x^{0}$ e $z^{0}$ interiores, o tamanho do passo $\alpha$ é calculado de forma que $x^{k+1}$ e $z^{k+1}$ permaneçam interiores (y é livre). Este método não necessita de um ponto inicial factivel.

\section{Critério de Convergência}

(i) Factibilidade primal: $\frac{\|b-A x\|}{\|b\|+1} \leq \epsilon$;

(ii) Factibilidade dual: $\frac{\left\|c-A^{t} y-z\right\|}{\|c\|+1} \leq \epsilon$;

(iii) Otimalidade - Gap relativo: $\frac{\left|c^{t} x-b^{t} y\right|}{1+c^{t} x+b^{t} y} \leq \epsilon$ ou $\left|\frac{x^{t} z}{1+c^{t} x+b^{t} y}\right| \leq \epsilon$. 


\section{Ponto Inicial}

Para o problema primal, temos [16]:

$$
\begin{aligned}
\tilde{x} & =A^{t}\left(A A^{t}\right)^{-1} b \Rightarrow A \tilde{x}=b, \\
x_{i}^{0} & =\max \left\{\tilde{x}_{i}, \epsilon_{1}\right\}, \\
\epsilon_{1} & =\max \left\{-\min \tilde{x}_{i}, \epsilon_{2}, \frac{\|b\|_{1}}{\epsilon_{2}\|A\|_{1}}\right\} \\
\epsilon_{2} & =100 .
\end{aligned}
$$

Agora, para o problema dual:

$$
\begin{aligned}
& y^{0}=0 \\
& z_{i}^{0}= \begin{cases}c_{i}+\epsilon_{3} & \text { se } c_{i} \geq 0 \\
-c_{i} & \text { se } c_{i} \leq-\epsilon_{3} \\
\epsilon_{3} & \text { se }-\epsilon_{3} \leq c_{i} \leq 0\end{cases} \\
& \epsilon_{3}=1+\|c\|_{1} .
\end{aligned}
$$

\subsubsection{Método Primal-Dual Clássico}

O método primal-dual afim-escala não é um método eficiente porque permite que alguns produtos $x_{i} z_{i}$ se aproximem de zero muito rapidamente [30]. Consequentemente, as direções calculadas nestas condições são muito distorcidas e o método progride lentamente, podendo inclusive não convergir.

Para evitar esta dificuldade, é acrescentada uma perturbação $\mu$ às condições de complementaridade $[16,30]$. No lugar de $x_{i} z_{i}=0$ temos agora $x_{i} z_{i}=\mu, i=1, \ldots, n$.

No método primal-dual resolvemos o seguinte sistema não-linear

$$
\left\{\begin{array}{cc}
A x=b, & x \geq 0 \\
A^{t} y+z=c, & z \geq 0 \\
X Z e=\mu e . &
\end{array}\right.
$$


Aplicando o método de Newton temos o seguinte sistema linear

$$
\left(\begin{array}{ccc}
A & 0 & 0 \\
0 & A^{t} & I \\
Z & 0 & X
\end{array}\right)\left(\begin{array}{l}
d x \\
d y \\
d z
\end{array}\right)=\left(\begin{array}{c}
r_{p} \\
r_{d} \\
r_{c}
\end{array}\right)=\left(\begin{array}{c}
b-A x \\
c-A^{t} y-z \\
\mu e-X Z e
\end{array}\right) .
$$

Podemos calcular as direções exatamente como no método primal-dual afim-escala, basta substituir $r_{a}$ por $r_{c}$. Em particular, o Jacobiano é o mesmo.

\section{Método primal-dual clássico}

Dados $\tau, \sigma \in(0,1)$ e $\left(x^{0}, y^{0}, z^{0}\right)$ interior ou $\left(x^{0}, z^{0}\right)>0$

Para $\mathrm{k}=0,1, \ldots$, faça

$$
\begin{aligned}
\mu^{k} & =\sigma \frac{\gamma^{k}}{n} \\
r_{p}^{k} & =b-A x^{k} \\
r_{d}^{k} & =c-A^{t} y^{k}-z^{k} \\
r_{c}^{k} & =\mu^{k} e-X^{k} Z^{k} e \\
d y^{k} & =\left[A\left(D^{k}\right)^{-1} A^{t}\right]^{-1}\left[r_{p}^{k}+A\left(D^{k}\right)^{-1} r_{d}^{k}-A\left(Z^{k}\right)^{-1} r_{c}^{k}\right] \\
d x^{k} & =\left(D^{k}\right)^{-1}\left[A^{t} d y^{k}-r_{d}^{k}+X^{-1} r_{c}^{k}\right] \\
d z^{k} & =\left(X^{k}\right)^{-1}\left[r_{c}^{k}-Z^{k} d x^{k}\right] \\
\rho_{p} & =\min _{d x_{i}^{k}<0}\left\{-\frac{x_{i}^{k}}{d x_{i}^{k}}\right\} \\
\rho_{d} & =\min _{d z_{i}^{k}<0}\left\{-\frac{z_{i}^{k}}{d z_{i}^{k}}\right\} \\
\alpha_{p}^{k} & =\min \left\{1, \tau \rho_{p}^{k}\right\} \\
\alpha_{d}^{k} & =\min \left\{1, \tau \rho_{d}^{k}\right\} \\
x^{k+1} & =x^{k}+\alpha_{p}^{k} d x^{k} \\
y^{k+1} & =y^{k}+\alpha_{d}^{k} d y^{k} \\
z^{k+1} & =z^{k}+\alpha_{d}^{k} d z^{k}
\end{aligned}
$$


Até convergir.

Observação 2.3.2 Se tomarmos $\mu^{k}=0$ temos o método afim-escala. Considere $\sigma=\frac{1}{\sqrt{n}}$ e $\tau=0$, 99995. Temos que $\gamma$ se reduz a cada iteração, portanto $\mu$ se reduz a cada iteração. Assim, quando $k \rightarrow \infty, \mu \rightarrow 0$.

Observação 2.3.3 Dependendo da escolha de $\tau$ e $\sigma$ obtemos resultados teóricos e práticos com respeito à eficiência do método. Uma variação importante ocorre quando $\gamma^{k}<1$. Neste caso utiliza-se $\mu^{k}=\sigma \frac{\left(\gamma^{k}\right)^{2}}{n}$. Existem motivações teóricas que justificam esta escolha [27].

\subsection{Método de Pontos Interiores Barreira Logarítmica}

Descreveremos agora o método de pontos interiores barreira logarítmica [3, 7] o qual utilizaremos para desenvolver o nosso método aplicado ao problema de regressão pela norma $L_{p}$.

Seja o problema de programação não linear:

$$
\begin{array}{r}
\min \quad f(x) \\
\text { sa } g(x) \leq 0 \\
h(x)=0 \\
x \in \Omega .
\end{array}
$$

Inserimos a variável de folga $s$ uma vez que neste método trabalhamos apenas com restrições de igualdade:

$$
\begin{array}{r}
\min \quad f(x) \\
\text { sa } g(x)+s=0 \\
h(x)=0 \\
s \geq 0 .
\end{array}
$$


Agora, relaxamos a restrição $s \geq 0$ inserindo-a na função objetivo através do parâmetro barreira $\mu, \operatorname{com} \mu \rightarrow 0$

$$
\begin{array}{r}
\min f(x)-\mu \sum_{i=1}^{n} \ln \left(s_{i}\right) \\
\text { sa } g(x)+s=0 \\
h(x)=0
\end{array}
$$

A função Lagrangiana é dada por

$$
L=f(x)-\mu \sum_{i=1}^{n} \ln \left(s_{i}\right)+\pi^{t}(g(x)+s)+\lambda^{t} h(x),
$$

onde $\pi$ e $\lambda$ são os multiplicadores de Lagrange.

Aplicando as condições de otimalidade, obtemos

$$
\underbrace{\nabla L}_{J(x, \pi, \lambda, s)}=\left[\begin{array}{c}
\nabla f(x)+\pi^{t} \nabla g(x)+\lambda^{t} \nabla h(x) \\
g(x)+s \\
h(x) \\
S \pi
\end{array}\right]=\left[\begin{array}{c}
0 \\
0 \\
0 \\
\mu e
\end{array}\right] .
$$

onde $S$ é a matriz diagonal cujos elementos diagonais são os elementos de $s$.

Utilizando o método de Newton, chegamos a

$$
\begin{gathered}
{\left[\begin{array}{cccc}
\nabla^{2} f(x)+\pi^{t} \nabla^{2} g(x)+\lambda^{t} \nabla^{2} h(x) & \nabla g(x) & \nabla h(x) & 0 \\
\nabla g(x) & 0 & 0 & I \\
\nabla h(x) & 0 & 0 & 0 \\
0 & S & 0 & \pi
\end{array}\right]\left[\begin{array}{c}
d x \\
d \pi \\
d \lambda \\
d s
\end{array}\right]=} \\
=-\left[\begin{array}{c}
\nabla f(x)+\pi^{t} \nabla g(x)+\lambda^{t} \nabla h(x) \\
g(x)+s \\
h(x) \\
-\mu e+S \pi
\end{array}\right] .
\end{gathered}
$$

Assim, obtemos as direções $d x, d \pi, d \lambda$ e $d s$. 
Agora, calculamos os passos $\alpha_{p}$ e $\alpha_{d}$, correspondentes as variáveis primais e duais respectivamente, onde $s$ e $\pi$ permaneçam estritamente positivas. Os passos $\alpha_{p}$ e $\alpha_{d}$ são calculados a fim de preservarem a interioridade de todas as variáveis restritas do problema.

Sejam

$$
\begin{array}{r}
\rho_{p}=\min _{d s_{i}<0}\left\{-\frac{s_{i}}{d s_{i}}\right\} \\
\mathrm{e} \\
\rho_{d}=\min _{d \pi_{i}<0}\left\{-\frac{\pi_{i}}{d \pi_{i}}\right\} .
\end{array}
$$

Assim, o passo $\alpha$ é dado por:

$$
\alpha=\min \left\{1, \tau \rho_{p}, \tau \rho_{d}\right\}
$$

Conhecendo as direções e os passos, todas as variáveis do problema podem ser atualizadas por:

$$
\begin{gathered}
x^{k+1}=x^{k}+\alpha d x, \\
s^{k+1}=s^{k}+\alpha d s, \\
\pi^{k+1}=\pi^{k}+\alpha d \pi, \\
\lambda^{k+1}=\lambda^{k}+\alpha d \lambda .
\end{gathered}
$$

A atualização do parâmetro barreira é dada por

$$
\mu^{k+1}=\frac{\mu^{k}}{\beta}, \quad \text { onde } \beta>1
$$

Resumimos agora o método barreira logarítmica.

\section{Método barreira logarítmica}


Dados $x$ interior, $(s, \pi)>0, \lambda, \mu$ e $\beta>1$.

Para $k=0,1, \ldots$, faça

Calcule o vetor gradiente da Lagrangiana (2.8).

Calcule a matriz Hessiana e resolva o sistema (2.9).

Calcule o passo $\alpha$ dado por (2.10).

Atualize as variáveis $(x, s, \pi, \lambda)$ e o parâmetro barreira $\mu$ dados por $(2.11)$ e (2.12) respectivamente.

Até convergir.

\subsubsection{Critério de Convergência}

O critério de convergência é dado por:

$$
\|\nabla L\|<\epsilon
$$

onde $\nabla L$ é dado por (2.8).

\subsubsection{Inicialização}

Considere o problema original na forma

$$
\begin{array}{r}
\min f(x)-\mu \sum \ln (s) \\
\text { sa } g(x)+s=0 \\
h(x)=0 .
\end{array}
$$

Inicialize o vetor $x$, as variáveis de folga $s>0$ tal que $g(x)+s=0$, o parâmetro barreira $\mu$, o parâmetro $\beta$ e os multiplicadores de Lagrange, onde $\pi=\mu S^{-1} e$. Escolha o valor do critério de parada $\epsilon$. 


\subsection{Método de Pontos Interiores Barreira Logarítmica Preditor-Corretor}

Descreveremos agora o método de pontos interiores barreira logarítmica preditorcorretor [4] o qual também utilizaremos para desenvolver o método aplicado ao problema de regressão pela norma $L_{p}$.

Nos métodos de pontos interiores, resolvemos um sistema não linear dado pelas condições de otimalidade e este sistema pode ser resolvido pelo método de Newton. O método de Newton possui uma dificuldade, ele garante apenas convergência local, isto é, convergência a partir de um ponto inicial que está suficientemente próximo da solução. Para ampliar a região de convergência dos métodos de pontos interiores é necessário combiná-los com algum outro método que possua propriedades de convergência global satisfatórias.

Uma variante do método de Newton é dada por:

$$
\begin{gathered}
\hat{x}^{k}=x^{k}-\left(\nabla^{2} f\left(x^{k}\right)\right)^{-1} \nabla f\left(x^{k}\right) \\
x^{k+1}=\hat{x}^{k}-\left(\nabla^{2} f\left(x^{k}\right)\right)^{-1} \nabla f\left(\hat{x}^{k}\right) .
\end{gathered}
$$

Dado $x^{k}$, um passo regular de Newton é realizado para obter $\hat{x}^{k}$ e utilizando a mesma Hessiana obtém-se $x^{k+1}$ a partir de $\hat{x}^{k}$. Este método de Newton de dois passos quando aplicado às condições de otimalidade é conhecido como método preditor-corretor [4].

Temos que, no método barreira logarítmica apenas os termos lineares de primeira ordem são modelados. Agora, no método barreira logarítmica preditor-corretor, introduzimos correções não lineares na equação (2.8). Assim, o sistema (2.9) é modificado da 
seguinte forma:

$$
\begin{gathered}
{\left[\begin{array}{cccc}
\nabla^{2} f(x)+\pi^{t} \nabla^{2} g(x)+\lambda^{t} \nabla^{2} h(x) & \nabla g(x) & \nabla h(x) & 0 \\
\nabla g(x) & 0 & 0 & I \\
\nabla h(x) & 0 & 0 & 0 \\
0 & 0 & \pi
\end{array}\right]\left[\begin{array}{c}
d x \\
d \pi \\
d \lambda \\
d s
\end{array}\right]=} \\
=-\left[\begin{array}{c}
\nabla f(x)+\pi^{t} \nabla g(x)+\lambda^{t} \nabla h(x) \\
g(x)+s \\
h(x) \\
-\mu e+S \pi+d S d \Pi e
\end{array}\right]
\end{gathered}
$$

onde $d S$ e $d \Pi$ são matrizes diagonais cujos elementos são $d s$ e $d \pi$ respectivamente.

No método barreira logarítmica preditor-corretor, primeiramente tomamos um passo afim em que o parâmetro barreira $\mu=0$. Então, o parâmetro barreira e os termos de segunda ordem podem ser obtidos a partir dos resultados do passo afim, e o lado direito de (2.13) pode ser calculado.

A diferença fundamental entre o método barreira logarítmica e o método barreira logarítmica preditor-corretor está na forma de calcular a direção de busca, que é obtida resolvendo dois sistemas lineares em cada iteração, onde a matriz dos coeficientes é a mesma e os lados direitos são distintos.

Resolvemos primeiro o sistema

$$
\underbrace{\left[\begin{array}{cccc}
\nabla^{2} f(x)+\pi^{t} \nabla^{2} g(x)+\lambda^{t} \nabla^{2} h(x) & \nabla g(x) & \nabla h(x) & 0 \\
\nabla g(x) & 0 & 0 & I \\
\nabla h(x) & 0 & 0 & 0 \\
0 & S & 0 & \pi
\end{array}\right]}_{W}\left[\begin{array}{c}
\overline{d x} \\
\overline{d \pi} \\
\overline{d \lambda} \\
\overline{d s}
\end{array}\right]=
$$




$$
=-\left[\begin{array}{c}
\nabla f(x)+\pi^{t} \nabla g(x)+\lambda^{t} \nabla h(x) \\
g(x)+s \\
h(x) \\
S \pi
\end{array}\right]
$$

que equivale ao sistema linear (2.9) $\operatorname{com} \mu=0$.

Agora, resolvemos o segundo sistema linear

$$
\begin{aligned}
& \underbrace{\left[\begin{array}{cccc}
\nabla^{2} f(x)+\pi^{t} \nabla^{2} g(x)+\lambda^{t} \nabla^{2} h(x) & \nabla g(x) & \nabla h(x) & 0 \\
\nabla g(x) & 0 & 0 & I \\
\nabla h(x) & 0 & 0 & 0 \\
0 & S & 0 & \pi
\end{array}\right]}_{W}\left[\begin{array}{c}
d x \\
d \pi \\
d \lambda \\
d s
\end{array}\right]= \\
& =-\left[\begin{array}{c}
\nabla f(x)+\pi^{t} \nabla g(x)+\lambda^{t} \nabla h(x) \\
g(x)+s \\
h(x) \\
-\mu e+S \pi+\overline{d S} \overline{d \Pi} e
\end{array}\right] .
\end{aligned}
$$

Podemos observar que temos a mesma matriz nos dois sistemas lineares. A diferença entre eles está apenas no vetor do lado direito, ou seja, a presença do termo não linear $\overline{d S} \bar{d} \Pi$ e do parâmetro barreira $\mu$. Assim, a fatoração da matriz $W$ do sistema (2.14) não é afetada em (2.15).

O método barreira logarítmica preditor-corretor reduz o número de iterações em relação ao método barreira logarítmica, mas exige que dois sistemas lineares sejam resolvidos em cada iteração. No entanto, como a matriz é a mesma nestes dois sistemas, os cálculos utilizados para a decomposição são efetuados uma única vez.

O cálculo dos passos primais e duais, $\alpha_{p}$ e $\alpha_{d}$ respectivamente, assim como a atualização das variáveis são equivalentes aos apresentados na seção anterior para o método barreira logarítmica. 
Descreveremos agora um resumo do método barreira logarítmica preditor-corretor.

\section{Método barreira logarítmica preditor-corretor}

Dados $x$ interior, $(s, \pi)>0, \lambda, \beta>1$.

Para $\mathrm{k}=0,1, \ldots$, faça

Resolva o sistema (2.14).

Entre com o parâmetro barreira $\mu$ e as correções não lineares.

Resolva o sistema (2.15).

Calcule o passo $\alpha$ dado por (2.10).

Atualize as variáveis $(x, s, \pi, \lambda)$ e o parâmetro barreira $\mu$ dados por $(2.11)$ e (2.12) respectivamente.

Até convergir.

O critério de convergência e a inicialização são dados por (2.4.1) e (2.4.2) respectivamente. 


\section{Capítulo 3}

\section{O Problema de Regressão $L_{p}$}

Apresentamos neste capítulo o problema de regressão $L_{p}$ e o método proposto em [13] com o objetivo de compararmos com os métodos de pontos interiores que desenvolvemos para o mesmo problema.

\subsection{O Problema de Regressão pela Norma $L_{p}$}

O problema de regressão

$$
\min _{x \in \mathbb{R}^{m}}\|A x-b\|_{p}^{p}
$$

onde $A=\left[a_{1}, \ldots, a_{n}\right] \in \mathbb{R}^{m \times n}, b \in \mathbb{R}^{n}$ e $n>m$, tem inúmeras aplicações em diversas áreas de ciências e engenharias. As normas mais utilizadas são as normas $L_{1}, L_{2}$ e $L_{\infty}$. A norma $L_{2}$ é muito popular entre outros motivos por permitir uma solução direta. Por sua vez a norma $L_{1}$ permite diminuir o efeito de pontos discrepantes enquanto que a norma $L_{\infty}$ garante proteção contra o pior caso. Os dois últimos problemas podem ser formulados por programação linear e os métodos de pontos interiores aplicados a estes problemas permitem a exploração da estrutura matricial do problema de forma bastante eficiente $[20,21]$.

O problema é teoricamente interessante, pois é uma extensão de um problema de minimização diferenciável por partes quando $p=1$, de um problema de minimização 
diferenciável de 1a ordem (mas não diferenciável de 2 a ordem) quando $1<p<2$ e até um problema diferenciável de 2 a ordem quando $p=2$.

O objetivo deste trabalho consiste na aplicação de métodos de pontos interiores ao problema de regressão $L_{p}$

$$
\begin{array}{ll}
\text { minimize } & \|r\|_{p}^{p} \\
\text { sujeito a } & A x+r=b,
\end{array}
$$

onde $1<p<2$.

Este problema pode combinar as propriedades de regressão das normas 1 e 2 de forma apropriada para cada aplicação.

Definindo $r=u-v, u \geq 0$ e $v \geq 0$, podemos reescrever o problema (3.2) da seguinte forma:

$$
\begin{array}{ll}
\operatorname{minimize} & \sum_{i=1}^{n}\left(u_{i}+v_{i}\right)^{p} \\
\text { sujeito a } & A x+u-v=b, \quad(u, v) \geq 0 .
\end{array}
$$

Ao transformarmos o valor absoluto em diferença de variáveis não negativas, $\|r\|_{p}^{p}=$ $\sum_{i=1}^{n}\left|u_{i}-v_{i}\right|^{p}$, temos que incluir a restrição $U V e=0$. No entanto, essa restrição pode ser ignorada se fizermos $\|r\|_{p}^{p}=\sum_{i=1}^{n}\left|u_{i}-v_{i}\right|^{p}=\sum_{i=1}^{n}\left(u_{i}+v_{i}\right)^{p}$, pois sempre existe um ponto factível com valor da função objetivo menor ou igual tal que $u_{i}=0$ ou $v_{i}=0$ para $i=1, \ldots, n$, ou seja, $U V e=0$ é verificado na otimalidade. Por exemplo, dados os vetores $u$ e $v$, calculamos o mínimo e o máximo de seus elementos $\left(m_{1}=\min (u, v)\right.$ e $m_{2}=\max (u, v)$ respectivamente). Logo após, calculamos a diferença entre eles, ou seja, $m_{2}-m_{1}$ e assim teremos um vetor da diferença e um vetor nulo.

Com este modelo não é necessário trabalhar com valores absolutos e, portanto, temos uma função cuja primeira derivada é definida para qualquer ponto. Além disso, quando 
$p=1$ temos exatamente o modelo de regressão $L_{1}$ resultando em um problema de otimização linear.

\subsection{Métodos Pré-Existentes}

\subsubsection{Métodos de Relaxação por Coluna para o problema de norma mínima}

O método desenvolvido em [8] é um método de relaxação de coluna para calcular a solução da norma $L_{p}$ de um sistema de equações lineares inconsistentes. Atenções especiais são dadas em cada um dos casos: $p=1,1<p<2,2<p<\infty$ e $p=\infty$.

Neste artigo, é assumido que o sistema linear $A x=b$ é inconsistente e que a matriz $A$ é grande, esparsa e desestruturada. Neste caso, geralmente os elementos não nulos de $A$ são armazenados por linha, depois de uma ordenação por linha, ou por coluna, depois de uma ordenação por coluna. Consequentemente, é conveniente resolver o problema por um método de relaxação por linha ou por um método de relaxação por coluna. A iteração básica de um esquema de uma relaxação por linha (coluna) é percorrer as linhas (colunas) de $A$.

Este artigo concentra-se no método de relaxação por coluna. A iteração básica de cada método é composta de $n$ passos. No $j$-ésimo passo, para $j=1,2, \ldots, n$, somente $x_{j}$ é modificado na tentativa de reduzir o valor da função objetivo, enquanto todas as outras variáveis são mantidas fixas. Utiliza-se o método de relaxação de Gauss-Seidel para resolver a equação normal $A^{t} A x=A^{t} b$.

A situação é mais complicada quando $1<p<2$. Neste caso, a segunda derivada de $F(x)=\|A x-b\|_{p}$ não é definida nos pontos onde o vetor residual $A x-b$ tem componentes zero. A solução proposta neste artigo é substituir $F(x)$ por uma função de 
aproximação hiperbólica da forma

$$
H(x)=\left\{\sum_{i=1}^{m}\left[\left(a_{i}^{t} x-b_{i}\right)^{2}+\epsilon^{2}\right]^{p / 2}\right\}^{1 / p},
$$

onde $a_{i}^{t}$ denota a i-ésima linha de $A$ e $\epsilon$ é uma constante positiva.

Não realizamos experimentos computacionais com este método uma vez que somente uma variável é atualizada a cada iteração, devendo convergir muito lentamente. O artigo [8] não apresenta resultados numéricos.

\subsubsection{Método GNCS}

Vamos agora descrever o método desenvolvido em [13] para o problema de regressão pela norma $L_{p}$ como segue. O método, referido como GNCS, é um método de Newton globalizado que usa as condições de folgas complementares para o problema da norma $L_{p}$. O conteúdo e as notações desta seção estão de acordo com o artigo [13], com exceção da utilização da matriz $A$ no lugar de $A^{t}$.

Considere o problema de regressão (3.1). Sejam $r$ o vetor residual $r=A x-b$ e $\sigma=\operatorname{sgn}(r)$ o seu sinal. A função objetivo é denotada em termos de $r$ por $\phi(r)=\|r\|_{p}^{p}$ $(=\psi(x))$ e o gradiente $\nabla \phi(r)$, quando ele existe, é denotado por $g=p(|r|)^{p-1} \sigma$.

Para $1<p<2$, o método tradicional para resolver (3.1) é o método (IRLS) iterativo de quadrados mínimos [17]. As direções de descida utilizadas por este método são derivadas da equação não linear $\psi(x)=0$. Esta é a condição de otimalidade para (3.1) quando $1<p<2$ mas não quando $p=1$. Quando $p=1$ o progresso torna-se lento ao longo da direção de descida, pois não tentamos satisfazer as condições de otimalidade diretamente e, portanto, acredita-se que esta é a causa do desempenho insatisfatório do método IRLS quando p é próximo ou igual a 1.

Em cada iteração, o custo computacional do método GNCS é o mesmo do método IRLS: resolução um problema de quadrados mínimos. Experimentos numéricos verificam 
em [13] que o GNCS é mais rápido que o tradicional método IRLS quando $p$ é quase ou igual a 1 .

Em [13] também é apresentado um procedimento de busca linear que explora a estrutura da função objetivo e impede resíduo nulo em cada iteração. O método GNCS apresenta-se melhor que o método IRLS e se reduz ao método de Coleman e Li [6] quando $p=1$.

Sabemos que a norma $L_{p}$ é diferenciável e estritamente convexa para $1<p<\infty$ desde que $A$ tenha posto completo. Então, a solução ocorre no ponto onde o gradiente $\nabla \psi(x)=A^{t} g$ é nulo. Supomos que existe um ponto com $r_{i} \neq 0,1 \leq i \leq n$. Isto é equivalente a

$$
A^{t}(D)^{-2} r=0
$$

onde $D=\operatorname{diag}\left((|r|)^{(2-p) / 2}\right)$, pois (3.4) é a equação normal para o seguinte sistema de quadrados mínimos:

$$
(D)^{-1} A x=(D)^{-1} b
$$

Suponhamos que as linhas da matriz $Z$ constituem uma base para o espaço nulo de $A$, isto é, $A^{t} Z=0$. Podemos escrever (3.4) da seguinte forma equivalente

$$
g-Z^{t} w=0
$$

O número de equações é $n$, que é equivalente ao número de variáveis $(x, w)$ (note que $x \in \mathbb{R}^{m}$ e $w \in \mathbb{R}^{n-m}$ ).

Seja $D_{r}^{k}=\operatorname{diag}\left(\left|r^{k}\right|\right)$ e denote $\lambda^{k}=Z^{t} w^{k}$. Temos que

$$
g-Z^{t} w=p(|r|)^{p-1}-Z^{t} w=p(|A x-b|)^{p-1}-Z^{t} w=0 .
$$

Derivando em relação a $(x, w)$,

$$
p(p-1)(|A x-b|)^{p-2} A-Z^{t}=0 .
$$


Assim, para qualquer ponto $\left(x^{k}, w^{k}\right)$ o passo de Newton para a equação acima é definido por

$$
\begin{gathered}
{\left[p(p-1) \operatorname{diag}\left(\left|r^{k}\right|\right)^{p-2} A,-Z^{t}\right]\left[d x^{k}, d w^{k}\right]^{t}=-\left[g^{k}-\lambda^{k}\right]} \\
\Rightarrow A^{t}(p-1) \operatorname{diag}\left(\left|r^{k}\right|^{-1}\right) \operatorname{diag}\left(p\left|r^{k}\right|^{p-1}\right) A d x=\underbrace{A^{t} Z d w}_{=0}-A^{t} g^{k}+\underbrace{A^{t} \lambda^{k}}_{=0} .
\end{gathered}
$$

Assim, a direção de Newton para a variável $x$ é

$$
d x^{k}=-\frac{1}{p-1}\left(A^{t}\left(D_{r}^{k}\right)^{-1} \operatorname{diag}\left(\left|g^{k}\right|\right) A\right)^{-1} A^{t} g^{k} .
$$

Agora, condideramos o seguinte sistema não linear de equações

$$
D_{r}\left(g-Z^{t} w\right)=0
$$

Quando $p=1$ esta é a condição de folga complementar para uma solução e $\lambda$ é o vetor multiplicador dual. Quando $1<p<2$, (3.7) é a condição de otimalidade para (3.1) se $D_{r}$ é não singular.

Como a solução para (3.5) é sempre uma solução para (3.7) e uma solução para (3.7) é uma solução para (3.5) se para qualquer $r_{i}=0, \lambda_{i}=0$, podemos calcular uma solução de (3.1) satisfazendo (3.7) e a condição $\lambda_{i}=0$ se $r_{i}=0$.

Considerando (3.7), as condições de otimalidade para minimização suave $(p>1)$ e parte das condições de otimalidade para minimização não suave $(p=1)$. Dado que a função objetivo $\phi(r)$ torna-se quase não suave quando $p$ é próximo de um, Li [13] afirma que é melhor considerar (3.7) que (3.5). Já que estamos interessados no problema da norma $L_{p}$ para todo $1 \leq p<2$, usar a direção de Newton definido por (3.7) é mais apropriado que usar (3.5). 
Supomos agora que o Jacobiano de $D_{r}\left(g-Z^{t} w\right)$ existe para $\left(x^{k}, w^{k}\right)$ e é não singular. Seja

$$
D_{\lambda}^{k}=\operatorname{diag}\left(p \sigma^{k} \cdot * g^{k}-\sigma^{k} \cdot * \lambda^{k}\right)
$$

onde .* é a notação do Matlab que representa o produto dos componentes dos vetores.

Temos que

$$
D_{r}\left(g-Z^{t} w\right)=\operatorname{diag}\left(\left|r^{k}\right|\right)\left[p(|A x-b|)^{p-1}-Z^{t} w\right]
$$

Derivando $D_{r}\left(g-Z^{t} w\right)$ com relação a $\left(x^{k}, w^{k}\right)$, obtemos

$$
\begin{gathered}
{\left[p(|A x-b|)^{p-1}-Z^{t} w\right] A+\operatorname{diag}\left(\left|r^{k}\right|\right)\left[p(p-1) \operatorname{diag}\left(\left|r^{k}\right|\right)^{p-2} A-Z^{t}\right]} \\
\Rightarrow\left[p\left(\left|r^{k}\right|\right)^{p-1}-\lambda\right] A+\operatorname{diag}\left(\left|r^{k}\right|\right)\left[(p-1) \operatorname{diag}\left(p\left(\left|r^{k}\right|\right)^{p-1}\right) \operatorname{diag}\left(\left|r^{k}\right|\right)^{-1} A-Z^{t}\right] \\
\Rightarrow\left[g^{k}-\lambda+\operatorname{diag}\left(g^{k}(p-1)\right)\right] A-D_{r}^{k} Z^{t} \\
\Rightarrow \underbrace{\operatorname{diag}\left(g^{k} p-g^{k}+g^{k}-\lambda\right)}_{=D_{\lambda}^{k}} A-D_{r}^{k} Z^{t} .
\end{gathered}
$$

Então o passo de Newton para (3.7) é definido por

$$
\begin{gathered}
{\left[D_{\lambda}^{k} A,-D_{r}^{k} Z^{t}\right]\left[d x^{k}, d w^{k}\right]^{t}=-\left[D_{r}^{k}\left(g^{k}-\lambda^{k}\right)\right]} \\
\Rightarrow D_{\lambda}^{k} A d x^{k}-D_{r}^{k} Z^{t} d w^{k}-D_{r}^{k} \lambda^{k}=-D_{r}^{k} g^{k} \\
\Rightarrow A^{t} D_{\lambda}^{k} A d x^{k}-\underbrace{A^{t} D_{r}^{k}\left(Z^{t} d w^{k}+\lambda^{k}\right)}_{=0}=-A^{t} D_{r}^{k} g^{k} .
\end{gathered}
$$

Daqui obtemos

$$
A^{t}\left(D_{r}^{k}\right)^{-1} D_{\lambda}^{k} A d x^{k}=-A^{t} g^{k}
$$

ou, equivalentemente,

$$
d x^{k}=-\left(A^{t}\left(D_{r}^{k}\right)^{-1} D_{\lambda}^{k} A\right)^{-1} A^{t} g^{k}
$$

Foi demonstrado em [6] que, quando $p=1, A^{t}\left(D_{r}\right)^{-1} D_{\lambda} A$ é definida positiva na 
vizinhança da solução, sob algumas hipóteses não degeneradas.

Consideremos o caso em que $1<p<2$. Se não há resíduo nulo na solução, isto é, $\left|r^{*}\right|>0,\left(D_{r}^{*}\right)^{-1} D_{\lambda}^{*}$ é definida positiva desde que $D_{\lambda}^{*}=(p-1) \operatorname{diag}\left(\left|g^{*}\right|\right)$ e supomos que A tem posto completo. Assim $A^{t}\left(D_{r}^{k}\right)^{-1} D_{\lambda}^{k} A$ também é definida positiva quando $\left(x^{k}, w^{k}\right)$ aproxima-se de $\left(x^{*}, w^{*}\right)$. Portanto a direção de Newton $d x^{k}$ torna-se uma direção de descida para $\psi(x)$ na vizinhança da solução.

Se existe algum $r_{i}^{*}=0$, a matriz Jacobiana de (3.7) é singular na solução quando $1<p<2$ porque $g_{i}^{*}=\lambda_{i}^{*}=0$. No entanto, nesses pontos a matriz Jacobiana do sistema original (3.4) também não existe. Portanto, este problema não surge quando consideramos (3.7) no lugar de (3.5). Se existe um resíduo nulo na solução $x^{*}$ é difícil obter convergência quadrática, então obtemos a convergência linear.

Já que $A^{t}\left(D_{r}^{k}\right)^{-1} D_{\lambda}^{k} A$ não pode ser definida positiva distante de uma solução, a globalização do passo de Newton (3.9) é necessária.

Para $p=1$ em [6], o método de Newton é globalizado definindo uma matriz diagonal $D_{\theta}^{k}$ tal que $A^{t}\left(D_{r}^{k}\right)^{-1} D_{\theta}^{k} A$ muda de $A^{t}\left(D_{r}^{k}\right)^{-1} A$ para $A^{t}\left(D_{r}^{k}\right)^{-1} D_{\lambda}^{k} A$ próximo a uma solução e substituindo $D_{\lambda}^{k}$ por $D_{\theta}^{k}$ quando a direção é calculada por (3.8).

Assim, o passo pode ser considerado como solução da seguinte equação

$$
\left[D_{\theta}^{k} A,-D_{r}^{k} Z^{t}\right]\left[d x^{k}, d w^{k}\right]^{t}=-D_{r}^{k}\left(g^{k}-\lambda^{k}\right) .
$$

Portanto,

$$
d x^{k}=-\left(A^{t}\left(D_{r}^{k}\right)^{-1} D_{\theta}^{k} A\right)^{-1} A^{t} g^{k}
$$

Se uma variável de controle $0<\theta<1$ que mede a proximidade para a solução for usada, a matriz diagonal $D_{\theta}$ pode ser definida da seguinte maneira

$$
D_{\theta}^{k}=\left|\theta^{k} \operatorname{diag}\left(\sigma^{k} g^{k}\right)+\left(1-\theta^{k}\right) D_{\lambda}^{k}\right|=\operatorname{diag}\left(\left|g^{k}-\left(1-\theta^{k}\right) \lambda^{k}\right|\right)
$$


onde $D_{\lambda}^{k}=\operatorname{diag}\left(g^{k}-\lambda^{k}\right)$.

Portanto, $\theta^{k}$ mede a satisfação da condição de folga complementar e a viabilidade dual do problema da norma $L_{1}$

$$
\theta^{k}=\frac{\eta^{k}}{\gamma+\eta^{k}}
$$

onde $\eta^{k}=\max \left\{\max \left\{\frac{\left|D_{r}^{k}\left(g^{k}-\lambda^{k}\right)\right|}{\phi\left(r^{0}\right)}\right\}, \max \left\{\max \left\{\left|\lambda^{k}\right|-\left|g^{k}\right|, 0\right\}\right\}\right\}$ e $0<\gamma<1$ (na implementação $\gamma=0.99$ ), ou seja, $\eta^{k}$ é o máximo da violação da condição de folga complementar $\left(D_{r}(g-\lambda)=0\right)$ e da viabilidade dual $(|\lambda| \leq|g|)$. Note que $|g|=\left|p(|r|)^{p-1}\right|=e$ quando $p=1$. Neste caso, $\theta=0$ (ou $\eta=0$ ) é uma condição de otimalidade necessária e suficiente (para uma discussão mais detalhada veja [6]).

Agora consideramos o caso em que $1<p<2$. Sabemos que a direção definida pelo método IRLS leva a convergência global, então definimos uma matriz diagonal $D_{\theta}$ tal que a direção obtida trocando $D_{\lambda}$ por $D_{\theta}$ seja a mesma direção do método IRLS e que localmente converge para $D_{\lambda}$. Assim, $D_{\theta}$ é uma matriz diagonal cuja diagonal é uma combinação convexa dos componentes de $\operatorname{diag}\left(p g^{k}\right)$ e $D_{\lambda}$.

$$
\begin{aligned}
D_{\theta}^{k} & =\left|\operatorname{diag}\left(\theta^{k}\right) \operatorname{diag}\left(p \sigma^{k} g^{k}\right)+\operatorname{diag}\left(e-\theta^{k}\right) D_{\lambda}^{k}\right| \\
& =\operatorname{diag}\left(\left|p g^{k}-\left(e-\theta^{k}\right) \cdot * \lambda^{k}\right|\right)
\end{aligned}
$$

e

$$
\theta^{k}=\left(\eta^{k} e\right) \cdot /\left(\gamma\left|g^{k}\right|+\eta^{k} e\right)
$$

onde $0<\gamma<1, e^{t}=[1, \ldots, 1] \in \mathbb{R}^{n}$ e o escalar $\eta^{k}$ é definido em (3.14).

Quando $p=1$, (3.15) é igual ao definido em (3.13) e portanto $D_{\theta}^{k}$ definido por (3.16) é equivalente ao definido por (3.14). Além disso, $x$ é ótimo se e somente se existe $\lambda=Z^{t} w$ tal que $\eta=0$.

A matriz diagonal $D_{\theta}^{k}$ tem as seguintes propriedades. 
Lema 3.2.1 Suponha $0<\gamma<1$. Seja $D_{\theta}^{k}$ definido por (3.15). Então Do satisfaz

$$
(p-1) \operatorname{diag}\left(\left|g^{k}\right|\right) \leq\left|D_{\theta}^{k}\right| \leq(p+1) \operatorname{diag}\left(\left|g^{k}\right|\right)
$$

Demonstração: Pela definição (3.15)

$$
D_{\theta}^{k}=\operatorname{diag}\left(\left|p g^{k}-\left(e-\theta^{k}\right) \cdot * \lambda^{k}\right|\right)
$$

Da definição de $\theta(3.16)$

$$
\begin{gathered}
\eta^{k}\left(e-\theta^{k}\right)=\gamma \theta^{k} \cdot *\left|g^{k}\right| \\
\Rightarrow\left(\left|\lambda^{k}\right|-\left|g^{k}\right|\right) \cdot *\left(e-\theta^{k}\right) \leq \gamma \theta^{k} \cdot *\left|g^{k}\right| .
\end{gathered}
$$

Assim,

$$
\begin{aligned}
\left|\lambda^{k}\right| & \leq\left|g^{k}\right|+\gamma\left(\theta^{k} \cdot *\left|g^{k}\right|\right) \cdot /\left(e-\theta^{k}\right) \\
& \leq\left(\left(e-\theta^{k}\right) \cdot *\left|g^{k}\right|+\gamma \theta^{k} \cdot *\left|g^{k}\right|\right) \cdot /\left(e-\theta^{k}\right) \\
& \leq\left(\left(e-(1-\gamma) \theta^{k}\right) \cdot * g^{k}\right) \cdot /\left(e-\theta^{k}\right) .
\end{aligned}
$$

Portanto,

$$
(p-1) \operatorname{diag}\left(\left|g^{k}\right|\right) \leq\left|D_{\theta}^{k}\right| \leq(p+1) \operatorname{diag}\left(\left|g^{k}\right|\right)
$$

Definimos $\tau^{k}$ como sendo

$$
\tau^{k}=\max \left(\tau, 1-\frac{\eta^{k}}{\gamma+\eta^{k}}\right)
$$

com o objetivo de incluir uma medida de otimalidade para $p=1$. Note que, quando $\left\{\eta^{k}\right\}$ converge para zero, $\left\{\tau^{k}\right\}$ converge para um. Quando $p=1$ o procedimento da busca linear para GNCS é equivalente ao usado em [6].

Para IRLS $\check{\alpha}^{k}$ é uma constante $p-1$. Para GNCS com $d^{k}$ definido por $(3.12), \check{\alpha}^{k}$ muda em cada iteração. No entanto, ele é limitado entre $p-1$ e $p+1$ como indicado pelo seguinte lema. 
Lema 3.2.2 Supomos $d^{k}=A d x^{k}$, onde $d x^{k}$ é definido por (3.12). Então o tamanho do passo $\check{\alpha}^{k}$ definido por

$$
\check{\alpha}^{k}=-\frac{g^{k^{t}} d^{k}}{d^{k^{t}} \operatorname{diag}\left(p\left(\left|r^{k}\right|\right)^{p-2}\right) d^{k}}
$$

satisfaz

$$
p-1 \leq \check{\alpha}^{k} \leq p+1
$$

Demonstração: De (3.12),

$$
\begin{aligned}
d x^{k}= & -\left(A^{t}\left(D_{r}^{k}\right)^{-1} D_{\theta}^{k} A\right)^{-1} A^{t} g^{k} \Rightarrow A^{t}\left(D_{r}^{k}\right)^{-1} D_{\theta}^{k} A d x^{k}=-A^{t} g^{k} \\
& \Rightarrow g^{k}=-\left(D_{r}^{k}\right)^{-1} D_{\theta}^{k} A d x^{k} \Rightarrow g^{k}=-\left(D_{r}^{k}\right)^{-1} D_{\theta}^{k} d^{k}
\end{aligned}
$$

Assim,

$$
\check{\alpha}^{k}=-\frac{d^{k}\left(D_{r}^{k}\right)^{-1} D_{\theta}^{k} d^{k}}{d^{k} \operatorname{diag}\left(p\left(\left|r^{k}\right|\right)^{p-2}\right) d^{k}}
$$

De (3.17),

$$
(p-1) \frac{d^{k^{t}}\left(D_{r}^{k}\right)^{-1} \operatorname{diag}\left(\left|g^{k}\right|\right) d^{k}}{d^{k^{t}} \operatorname{diag}\left(p\left(\left|r^{k}\right|\right)^{p-2}\right) d^{k}} \leq \check{\alpha}^{k} \leq(p+1) \frac{d^{k^{t}}\left(D_{r}^{k}\right)^{-1} \operatorname{diag}\left(\left|g^{k}\right|\right) d^{k}}{d^{k^{t}} \operatorname{diag}\left(p\left(\left|r^{k}\right|\right)^{p-2}\right) d^{k}}
$$

Logo,

$$
p-1 \leq \check{\alpha}^{k} \leq p+1
$$

Computacionalmente, em vez de resolver um sistema linear $n \times n$ (3.11) para calcular $\left(d x^{k}, d w^{k}\right)$, pode-se preferir calcular $d x^{k}$ resolvendo o problema de quadrados mínimos $n \times m$

$$
\left(D^{k}\right)^{-1} A d x^{k}=-D^{k} g^{k}
$$

onde $D^{k}=\left(D_{r}^{k}\left(D_{\theta}^{k}\right)^{-1}\right)^{1 / 2}$. Portanto,

$$
\left\{\begin{array}{l}
A^{t}\left(D^{k}\right)^{-2} r=0 \\
A^{t}\left(D^{k}\right)^{-2} A d x^{k}=-A^{t} D^{k} g^{k} \\
d^{k}=A d x^{k}
\end{array}\right.
$$


Uma vez que $d^{k}=A d x^{k}$ é calculado, $\lambda$ pode ser atualizado por

$$
\lambda^{k+1} \leftarrow\left(D_{r}^{k}\right)^{-1} D_{\theta}^{k} d^{k}+g^{k} .
$$

O método GNCS pode ser resumido como segue.

Dado o ponto inicial $r^{0}=A x^{0}-b \operatorname{com}\left|r^{0}\right|>0$ e $\lambda^{0}$.

Passo1: Calcular $\theta^{k}$ por (3.16) e $g^{k}=p\left(\left|r^{k}\right|\right)^{p-1} \sigma^{k}$.

Sejam $D_{r}^{k}=\operatorname{diag}\left(\left|r^{k}\right|\right), D_{\theta}^{k}=\operatorname{diag}\left(\left|p g^{k}-\left(e-\theta^{k}\right) \cdot * \lambda^{k}\right|\right)$.

Defina $D^{k}=\left(D_{r}^{k}\left(D_{\theta}^{k}\right)^{-1}\right)^{1 / 2}$;

Passo2: Calcule a direção $d^{k}$ por

$$
\left\{\begin{array}{l}
A^{t}\left(D^{k}\right)^{-2} r=0, \\
A^{t}\left(D^{k}\right)^{-2} A d x^{k}=-A^{t} D^{k} g^{k} \\
d^{k}=A d x^{k}
\end{array}\right.
$$

Atualize $\lambda^{k+1}$ :

$$
\lambda^{k+1} \leftarrow\left(D_{r}^{k}\right)^{-1} D_{\theta}^{k} d^{k}+g^{k}
$$

Passo3: Calcule $\tau^{k}$ por (3.18).

Use o procedimento de busca linear descrito a seguir.

Atualize $r^{k+1} \leftarrow r^{k}+\alpha^{k} d^{k}, k \leftarrow k+1$.

Vá para o passo 1.

Observação 3.2.1 A desvantagem deste método é que a busca linear é cara.

\section{Procedimento de Busca Linear}

Descreveremos agora o procedimento de busca linear utilizado pelo método GNCS. 
$\operatorname{Dados} \tau^{k}, \beta_{f} \in(0,1), d^{k}, r^{k}, \check{\alpha}^{k}, \rho_{b}>0$ (p.ex. $\left.10^{6}\right)$ e $\alpha_{i}^{k}$ definido por

$$
\jmath=\left\{\alpha_{i}^{k}: \alpha_{i}^{k}=-\frac{r_{i}^{k}}{d_{i}^{k}}, r_{i}^{k} d_{i}^{k}<0\right\} .
$$

Passo1: Seja $\alpha_{*}^{k}=\min \left(r^{k}+\alpha_{i}^{k} d^{k}\right) \operatorname{com} g\left(r^{k}+\alpha_{*}^{k} d^{k}\right)^{t} d^{k} \geq 0$. Se

$$
\phi\left(r^{k+1}\right) \leq \phi\left(r^{k}\right)+\beta_{f} \alpha^{k} \nabla \phi\left(r^{k}\right)^{t} d^{k}
$$

onde $r^{k+1}=r^{k}+\alpha^{k} d^{k}$ é satisfeito com $\alpha_{*}^{k}$, seja $\alpha_{\#}^{k} \leftarrow \max \left\{\alpha_{i}^{k}: 0 \leq \alpha_{i}^{k}<\alpha_{*}^{k}\right\}$ e defina

$$
\alpha^{k} \leftarrow \alpha_{\#}^{k}+\tau^{k}\left(\alpha_{*}^{k}-\alpha_{\#}^{k}\right)
$$

e retorna; caso contrário, continua;

Passo 2: Se (3.22) não é satisfeito com $\alpha^{k}=1$, vá para o passo 3.

Caso contrário, estabeleça

$$
\alpha^{k} \leftarrow \begin{cases}1, & \text { se } \min \left(\left|r^{k}+d^{k}\right|\right)>0 ; \\ \alpha_{\#}^{k}+\tau^{k}\left(1-\alpha_{\#}^{k}\right), & \text { caso contrário, }\end{cases}
$$

onde $\alpha_{\#}^{k} \leftarrow \max \left\{\alpha_{i}^{k}: 0 \leq \alpha_{i}^{k}<1\right\}$, retorna;

Passo 3: Seja

$$
\alpha^{k} \leftarrow \begin{cases}\check{\alpha}^{k}, & \text { se min }\left(\left|r^{k}+\check{\alpha}^{k} d^{k}\right|\right)>0 \\ \alpha_{\#}^{k}+\tau^{k}\left(\check{\alpha}^{k}-\alpha_{\#}^{k}\right), & \text { caso contrário, }\end{cases}
$$

onde $\alpha_{\#}^{k} \leftarrow \max \left\{\alpha_{i}^{k}: 0 \leq \alpha_{i}^{k}<\check{\alpha}^{k}\right\}$, retorna.

\section{Critério de Convergência}

O critério de convergência utilizado em [13] é dado por:

$$
\frac{\left|\phi\left(r^{k+1}\right)-\phi\left(r^{k}\right)\right|}{\phi\left(r^{k+1}\right)}<\epsilon \quad \text { ou } \quad \eta^{k}<\epsilon .
$$

Com o objetivo de compararmos os resultados do método GNCS com os resultados dos 
nossos métodos de pontos interiores de uma forma mais eficiente, o critério de convergência utilizado neste trabalho será dado por:

$$
\frac{\left\|D_{r}^{k}\left(\lambda^{k}-g\right)\right\|}{\left\|r^{k}\right\|(2 n)} \leq \epsilon .
$$




\section{Capítulo 4}

\section{Métodos de Pontos Interiores}

\section{Aplicados ao Problema de Regressão pela Norma $L_{p}$}

Neste capítulo, desenvolvemos uma família de métodos de pontos interiores para o problema de regressão $L_{p}$ : o método barreira logarítmica, o método primal-dual e a variante preditor-corretor.

\subsection{Método Barreira Logarítmica}

O problema (3.3) também pode ser escrito como

$$
\begin{aligned}
& \min \sum_{i=1}^{n}\left(u_{i}+v_{i}\right)^{p} \\
& \text { sa } A x+u-v-b=0, \quad(u, v) \geq 0
\end{aligned}
$$

A função objetivo é denotada em termos de $u$ e $v$ por $\phi(u, v)=\sum_{i=1}^{n}\left(u_{i}+v_{i}\right)^{p}$, o gradiente $\nabla \phi(u, v)$ é denotado por

$$
G=\left[\begin{array}{l}
G_{u} \\
G_{v}
\end{array}\right]
$$


onde $G_{u_{i}}=G_{v_{i}}=p\left(u_{i}+v_{i}\right)^{p-1} \mathrm{e}$

$$
\nabla^{2} \phi=\left[\begin{array}{c}
\nabla G_{u} \\
\nabla G_{v}
\end{array}\right]
$$

onde $\nabla G_{u_{i j}}=\nabla G_{v_{i j}}=\left\{\begin{array}{ll}\frac{p(p-1)}{\left(u_{i}+v_{i}\right)^{2-p}}, & \text { se } i=j, \\ 0, & \text { se } i \neq j\end{array}\right.$ é uma matriz diagonal denotada por $G_{2}$

Como temos um problema de otimização não linear, usamos a forma padrão para desenvolver um método de pontos interiores: aplicamos o método de Newton às condições de otimalidade.

Assim, temos

$$
\begin{aligned}
& \min \sum_{i=1}^{n}\left(u_{i}+v_{i}\right)^{p}-\mu \sum_{i=1}^{n} \ln \left(u_{i}\right)-\mu \sum_{i=1}^{n} \ln \left(v_{i}\right) \\
& \text { sa } A x+u-v-b=0
\end{aligned}
$$

onde $\mu>0$ é o parâmetro barreira $(\mu \rightarrow 0)$.

A Lagrangiana é dada por

$$
L=\sum_{i=1}^{n}\left(u_{i}+v_{i}\right)^{p}-\mu \sum_{i=1}^{n} \ln \left(u_{i}\right)-\mu \sum_{i=1}^{n} \ln \left(v_{i}\right)+y^{t}(A x+u-v-b),
$$

onde $y$ é o multiplicador de Lagrange.

Aplicando as condições de otimalidade, obtemos

$$
\underbrace{\nabla L}_{J(x, y, u, v)}=\left[\begin{array}{c}
A^{t} y \\
A x+u-v-b \\
\left(G-\mu U^{-1}+Y\right) e \\
\left(G-\mu V^{-1}-Y\right) e
\end{array}\right]=\left[\begin{array}{l}
0 \\
0 \\
0 \\
0
\end{array}\right] .
$$

onde $U$ e $V$ são matrizes diagonais cujos elementos diagonais são $u$ e $v$ respectivamente. 
Reescrevendo as duas últimas equações de (4.2),

$$
\underbrace{\nabla L}_{J(x, y, u, v)}=\left[\begin{array}{c}
A^{t} y \\
A x+u-v-b \\
U(G+Y) e \\
V(G-Y) e
\end{array}\right]=\left[\begin{array}{c}
0 \\
0 \\
\mu e \\
\mu e
\end{array}\right] .
$$

Utilizando o Método de Newton, chegamos a

$$
\left[\begin{array}{cccc}
0 & A^{t} & 0 & 0 \\
A & 0 & I & -I \\
0 & U & G+Y+U G_{2} & U G_{2} \\
0 & -V & V G_{2} & G-Y+V G_{2}
\end{array}\right]\left[\begin{array}{c}
d x \\
d y \\
d u \\
d v
\end{array}\right]=\left[\begin{array}{c}
r_{1} \\
r_{2} \\
r_{3} \\
r_{4}
\end{array}\right]
$$

onde

$$
\begin{aligned}
& r_{1}=-A^{t} y, \\
& r_{2}=-A x-u+v+b, \\
& r_{3}=-U(G+Y) e+\mu e \quad \mathrm{e} \\
& r_{4}=-V(G-Y) e+\mu e .
\end{aligned}
$$

Resolvendo o sistema (4.3), obtemos as direções $d x, d y, d u, d v$. Assim, temos o sistema

$$
\begin{aligned}
A^{t} d y & =r_{1} \\
A d x+d u-d v & =r_{2} \\
U d y+\left[G+Y+U G_{2}\right] d u+U G_{2} d v & =r_{3} \\
-V d y+V G_{2} d u+\left[G-Y+V G_{2}\right] d v & =r_{4} .
\end{aligned}
$$

Da Equação (4.6),

$$
\begin{gathered}
U d y+\left[G+Y+U G_{2}\right] d u+U G_{2} d v=r_{3} \\
\Rightarrow\left[G+Y+U G_{2}\right] d u=r_{3}-U d y-U G_{2} d v \\
\Rightarrow d u=\left[G+Y+U G_{2}\right]^{-1}\left(r_{3}-U d y-U G_{2} d v\right) .
\end{gathered}
$$


Fazendo $D_{u}=\left[G+Y+U G_{2}\right]$, obtemos

$$
d u=D_{u}^{-1}\left(r_{3}-U d y-U G_{2} d v\right) .
$$

Agora, da Equação (4.7),

$$
\begin{gathered}
-V d y+V G_{2} d u+\left[G-Y+V G_{2}\right] d v=r_{4} \\
\Rightarrow\left[G-Y+V G_{2}\right] d v=r_{4}+V d y-V G_{2} d u \\
\Rightarrow\left[G-Y+V G_{2}\right] d v=r_{4}+V d y+D_{u}^{-1}\left(-V G_{2} r_{3}+V U G_{2} d y+V U G_{2}^{2} d v\right) \\
\Rightarrow\left[G-Y+V G_{2}-V U G_{2}^{2} D_{u}^{-1}\right] d v=r_{4}+V d y+D_{u}^{-1}\left(-V G_{2} r_{3}+V U G_{2} d y\right) \\
\Rightarrow d v=\left[G-Y+V G_{2}-V U G_{2}^{2} D_{u}^{-1}\right]^{-1}\left[r_{4}+V d y+D_{u}^{-1}\left(-V G_{2} r_{3}+V U G_{2} d y\right)\right] .
\end{gathered}
$$

Fazendo $D_{v}=\left[G-Y+V G_{2}\right]$, obtemos

$$
d v=\left[D_{v}-V U G_{2}^{2} D_{u}^{-1}\right]^{-1}\left[r_{4}+V d y+D_{u}^{-1}\left(-V G_{2} r_{3}+V U G_{2} d y\right)\right]
$$

Da Equação (4.5),

$$
\begin{gathered}
A d x+d u-d v=r_{2} \\
\Rightarrow A d x+D_{u}^{-1}\left(r_{3}-U d y-U G_{2} d v\right)-\left[D_{v}-V U G_{2}^{2} D_{u}^{-1}\right]^{-1}\left[r_{4}+V d y+D_{u}^{-1}\left(-V G_{2} r_{3}+V U G_{2} d y\right)\right]=r_{2} \\
\Rightarrow \quad A d x+D_{u}^{-1}\left\{r_{3}-U d y-U G_{2}\left[D_{v}-V U G_{2}^{2} D_{u}^{-1}\right]^{-1}\left[r_{4}+V d y+D_{u}^{-1}\left(-V G_{2} r_{3}+V U G_{2} d y\right)\right]\right\}+ \\
\quad-\quad\left[D_{v}-V U G_{2}^{2} D_{u}^{-1}\right]^{-1}\left[r_{4}+V d y+D_{u}^{-1}\left(-V G_{2} r_{3}+V U G_{2} d y\right)\right]=r_{2} \\
\Rightarrow \quad-\quad D_{u}^{-1} U d y-D_{u}^{-1} U G_{2}\left[D_{v}-V U G_{2}^{2} D_{u}^{-1}\right]^{-1}\left[\left(V+D_{u}^{-1} V U G_{2}\right) d y\right]-\left[D_{v}-V U G_{2}^{2} D_{u}^{-1}\right]^{-1} \times \\
\times \quad\left[\left(V+D_{u}^{-1} V U G_{2}\right) d y\right]=r_{2}-A d x-D_{u}^{-1}\left\{r_{3}-U G_{2}\left[D_{v}-V U G_{2}^{2} D_{u}^{-1}\right]^{-1}\left[r_{4}-D_{u}^{-1} V G_{2} r_{3}\right]\right\} \\
+\quad\left[D_{v}-V U G_{2}^{2} D_{u}^{-1}\right]^{-1}\left(r_{4}-D_{u}^{-1} V G_{2} r_{3}\right) \\
\Rightarrow \quad\left\{-D_{u}^{-1} U-\left[D_{v}-V U G_{2}^{2} D_{u}^{-1}\right]^{-1} V\left(I+D_{u}^{-1} U G_{2}\right)^{2}\right\} d y=r_{2}-A d x-D_{u}^{-1} r_{3}+ \\
\quad+\left[D_{v}-V U G_{2}^{2} D_{u}^{-1}\right]^{-1}\left(I+D_{u}^{-1} U G_{2}\right)\left(r_{4}-D_{u}^{-1} V G_{2} r_{3}\right)
\end{gathered}
$$




$$
\begin{aligned}
\Rightarrow d y & =\left\{-D_{u}^{-1} U-\left[D_{v}-V U G_{2}^{2} D_{u}^{-1}\right]^{-1} V\left(I+D_{u}^{-1} U G_{2}\right)^{2}\right\}^{-1}\left\{r_{2}-A d x-D_{u}^{-1} r_{3}+\right. \\
& \left.+\left[D_{v}-V U G_{2}^{2} D_{u}^{-1}\right]^{-1}\left(I+D_{u}^{-1} U G_{2}\right)\left(r_{4}-D_{u}^{-1} V G_{2} r_{3}\right)\right\} .
\end{aligned}
$$

Fazendo $D=\left\{-D_{u}^{-1} U-\left[D_{v}-V U G_{2}^{2} D_{u}^{-1}\right]^{-1}\left[V\left(I+D_{u}^{-1} U G_{2}\right)\right]^{2}\right\}^{-1}$, temos $d y=D\left\{r_{2}-A d x-D_{u}^{-1} r_{3}+\left[D_{v}-V U G_{2}^{2} D_{u}^{-1}\right]^{-1}\left(I+D_{u}^{-1} U G_{2}\right)\left(r_{4}-D_{u}^{-1} V G_{2} r_{3}\right)\right\}$.

Finalmente, de (4.4), obtemos

$$
\begin{gathered}
A^{t} d y=r_{1} \\
\Rightarrow A^{t} D\left\{r_{2}-A d x-D_{u}^{-1} r_{3}+\left[D_{v}-V U G_{2}^{2} D_{u}^{-1}\right]^{-1}\left(I+D_{u}^{-1} U G_{2}\right)\left(r_{4}-D_{u}^{-1} V G_{2} r_{3}\right)\right\}=r_{1} \\
\Rightarrow-A^{t} D A d x=r_{1}-A^{t} D\left\{r_{2}-D_{u}^{-1} r_{3}+\left[D_{v}-V U G_{2}^{2} D_{u}^{-1}\right]^{-1}\left(I+D_{u}^{-1} U G_{2}\right)\left(r_{4}-D_{u}^{-1} V G_{2} r_{3}\right)\right\} \\
\Rightarrow d x=\left(-A^{t} D A\right)^{-1}\left\{r_{1}-A^{t} D\left[r_{2}-D_{u}^{-1} r_{3}+\left[D_{v}-V U G_{2}^{2} D_{u}^{-1}\right]^{-1}\left(I+D_{u}^{-1} U G_{2}\right)\left(r_{4}-D_{u}^{-1} V G_{2} r_{3}\right)\right]\right\}
\end{gathered}
$$

Definindo $r=-\left\{r_{1}-A^{t} D\left[r_{2}-D_{u}^{-1} r_{3}+\left[D_{v}-V U G_{2}^{2} D_{u}^{-1}\right]^{-1}\left(I+D_{u}^{-1} U G_{2}\right)\left(r_{4}-D_{u}^{-1} V G_{2} r_{3}\right)\right]\right\}$ obtemos

$$
d x=\left(A^{t} D A\right)^{-1} r .
$$

Agora, vamos calcular o passo $\alpha$, onde $u$ e $v$ permaneçam estritamente positivas.

$$
\alpha=\min \left\{\tau\left(\min _{d u_{i}<0}-\frac{u_{i}}{d u_{i}}\right), \tau\left(\min _{d v_{i}<0}-\frac{v_{i}}{d v_{i}}\right), 1\right\},
$$

onde $\tau=0.9995$ [30].

Conhecendo as direções e os passos, todas as variáveis do problema podem ser atualizadas por

$$
\begin{aligned}
& x^{k+1}=x^{k}+\alpha d x, \\
& u^{k+1}=u^{k}+\alpha d u, \\
& v^{k+1}=v^{k}+\alpha d v, \\
& y^{k+1}=y^{k}+\alpha d y .
\end{aligned}
$$


A atualização do parâmetro barreira é dada por

$$
\mu^{k+1}=\frac{\mu^{k}}{\beta}, \quad \text { onde } \beta>1 .
$$

Apresentamos agora o resumo do método de pontos interiores barreira logarítmica para o problema $L_{p}$.

$\operatorname{Dados} x^{0}, y^{0}$ e $\left(u^{0}, v^{0}\right)>0, \mu^{0}$ e $\beta>1$.

Para $k=0,1,2, \ldots$ faça

$$
\begin{aligned}
& G=p\left(u_{i}+v_{i}\right)^{p-1} \\
& G_{2}=\operatorname{diag}\left(\frac{p(p-1)}{\left(u_{i}+v_{i}\right)^{2-p}}\right) \\
& r_{1}^{k}=-A^{t} y \\
& r_{2}^{k}=-A x-u+v+b \\
& r_{3}=-U(G+Y) e+\mu e \\
& r_{4}=-V(G-Y) e+\mu e \\
& D_{u}=\left[G+Y+U G_{2}\right] \\
& D_{v}=\left[G-Y+V G_{2}\right] \\
& D=\left\{D_{u}{ }^{-1} U+\left[D_{v}-V U G_{2}^{2} D_{u}{ }^{-1}\right]^{-1} V\left(I+D_{u}{ }^{-1} U G_{2}\right)^{2}\right\}^{-1} \\
& r=\left\{r_{1}-A^{t} D\left[r_{2}-D_{u}{ }^{-1} r_{3}+\left[D_{v}-V U G_{2}^{2} D_{u}{ }^{-1}\right]^{-1}\left(I+D_{u}{ }^{-1} U G_{2}\right)\left(r_{4}-D_{u}{ }^{-1} V G_{2} r_{3}\right)\right]\right\} \\
& d x=\left(A^{t} D A\right)^{-1} r \\
& d y=D\left\{r_{2}-A d x-D_{u}^{-1} r_{3}+\left[D_{v}-V U G_{2}^{2} D_{u}^{-1}\right]^{-1}\left(I+D_{u}{ }^{-1} U G_{2}\right)\left(r_{4}-D_{u}{ }^{-1} V G_{2} r_{3}\right)\right\} \\
& d v=\left[D_{v}-V U G_{2}^{2} D_{u}^{-1}\right]^{-1}\left[r_{4}+V d y+D_{u}^{-1}\left(-V G_{2} r_{3}+V U G_{2} d y\right)\right] \\
& d u=r D_{u}^{-1}\left(r_{3}-U d y-U G_{2} d v\right) \\
& \alpha=\min \left\{\tau\left(\min _{d u_{i}<0}-\frac{u_{i}}{d u_{i}}\right), \tau\left(\min _{d v_{i}<0}-\frac{v_{i}}{d v_{i}}\right), 1\right\} \\
& u^{k+1}=u^{k}+\alpha d u \\
& v^{k+1}=v^{k}+\alpha d v
\end{aligned}
$$




$$
\begin{aligned}
x^{k+1} & =x^{k}+\alpha d x \\
y^{k+1} & =y^{k}+\alpha d y \\
\mu^{k+1} & =\frac{\mu^{k}}{\beta}
\end{aligned}
$$

Até convergir.

\subsubsection{Critério de Convergência}

O critério de convergência é baseado nas condições de otimalidade (4.2):

$$
N=\frac{\|\nabla L\|}{(1+\|x\|+\|u\|+\|v\|+\|y\|)(2 n)} \leq \epsilon
$$

Utilizamos também um critério de convergência baseado na diferença dos valores de $N$ atual e da iteração anterior:

$$
\left|N^{k+1}-N^{k}\right| \leq \epsilon_{1}
$$

\subsubsection{Pontos Iniciais}

Os pontos iniciais são calculados baseados nas idéias desenvolvidas em [6],

$$
\begin{aligned}
x^{0} & =\left(A^{t} A\right)^{-1} A^{t} b, \\
r^{0} & =b-A x^{0}, \\
u_{i}^{0} & = \begin{cases}\frac{\lambda+1}{2} r_{i}^{0} & \text { se } r_{i}^{0}>0, \\
-\frac{\lambda-1}{2} r_{i}^{0} & \text { caso contrário, }\end{cases} \\
v_{i}^{0} & = \begin{cases}\frac{\lambda-1}{2} r_{i}^{0} & \text { se } r_{i}^{0}>0, \\
-\frac{\lambda+1}{2} r_{i}^{0} & \text { caso contrário, }\end{cases} \\
y^{0} & =\frac{\kappa r^{0}}{\left\|r^{0}\right\|_{\infty}} .
\end{aligned}
$$

De fato, em [6] $u$ e $v$ não são definidos. Foi proposto em [21] esta escolha de modo que a relação $u^{0}+v^{0}=\left|\lambda r^{0}\right|$ seja satisfeita. Assim, se $\lambda$ é $O(1)$, ambos $u^{0}$ e $v^{0}$ são da mesma ordem de $r^{0}$. Lembramos que, por definição, $r=u-v$. Estabelecemos $\kappa=0,975$. 


\subsubsection{Algumas Considerações}

Na maioria dos métodos de pontos interiores o passo mais caro em termos computacionais é a solução do sistema linear com uma matriz do tipo $A^{t} D A$, onde $D$ é uma matriz diagonal.

O sistema linear obtido em nosso trabalho é simétrico e definido positivo e assim pode ser resolvido pela fatoração de Cholesky. Obtemos, então, um sistema muito menor que o sistema original (4.3) e da mesma dimensão dos sistemas resolvidos pelo método GNCS [13]. Vale lembrar que, nessa classe de problemas, $A$ é uma matriz cheia.

\subsection{Método Preditor-Corretor}

Neste método, utilizaremos a mesma formulação do problema apresentado na seção (4.1).

No método preditor-corretor, primeiramente tomamos a direção afim em que o parâmetro barreira $\mu=0$. Assim, temos que resolver o sistema

$$
\left[\begin{array}{cccc}
0 & A^{t} & 0 & 0 \\
A & 0 & I & -I \\
0 & U & G+Y+U G_{2} & U G_{2} \\
0 & -V & V G_{2} & G-Y+V G_{2}
\end{array}\right]\left[\begin{array}{c}
\overline{d x} \\
\overline{d y} \\
\overline{d u} \\
\overline{d v}
\end{array}\right]=\left[\begin{array}{c}
\overline{r_{1}} \\
\overline{r_{2}} \\
\overline{r_{3}} \\
\overline{r_{4}}
\end{array}\right]
$$

onde

$$
\begin{aligned}
& \overline{r_{1}}=-A^{t} y, \\
& \overline{r_{2}}=-A x-u+v+b, \\
& \overline{r_{3}}=-U(G+Y) e \mathrm{e} \\
& \overline{r_{4}}=-V(G-Y) e,
\end{aligned}
$$

que é o mesmo sistema dado pela Equação (4.3) $\operatorname{com} \mu=0$. 
Resolvendo o sistema (4.8), obtemos as direções afim $\overline{d x}, \overline{d y}, \overline{d u}, \overline{d v}$. Assim, temos o sistema

$$
\begin{aligned}
A^{t} \overline{d y} & =\overline{r_{1}} \\
A \overline{d x}+\overline{d u}-\bar{d} v & =\overline{r_{2}} \\
U \overline{d y}+\left[G+Y+U G_{2}\right] \overline{d u}+U G_{2} \overline{d v} & =\overline{r_{3}} \\
-V \overline{d y}+V G_{2} \overline{d u}+\left[G-Y+V G_{2}\right] \overline{d v} & =\overline{r_{4}} .
\end{aligned}
$$

Fazendo a mesma eliminação de variáveis utilizada no método barreira logarítmica, obtemos as direções

$$
\begin{aligned}
\overline{d x} & =\left(A^{t} \bar{D} A\right)^{-1} \bar{r} \\
\overline{d y} & =\bar{D}\left\{\overline{r_{2}}-A \overline{d x}-{\overline{D_{u}}}^{-1} \overline{r_{3}}+\left[\overline{D_{v}}-V U G_{2}^{2}{\overline{D_{u}}}^{-1}\right]^{-1}\left(I+\bar{D}_{u}^{-1} U G_{2}\right)\left(\bar{r}_{4}-\bar{D}_{u}{ }^{-1} V G_{2} \bar{r}_{3}\right)\right\}, \\
\overline{d v} & =\left[\bar{D}_{v}-V U G_{2}^{2} \bar{D}_{u}^{-1}\right]^{-1}\left[\overline{r_{4}}+V \overline{d y}+{\overline{D_{u}}}^{-1}\left(-V G_{2} \bar{r}_{3}+V U G_{2} \overline{d y}\right)\right], \\
\overline{d u} & =\bar{D}_{u}{ }^{-1}\left(\overline{r_{3}}-U \overline{d y}-U G_{2} \overline{d v}\right),
\end{aligned}
$$

onde

$$
\begin{aligned}
\overline{r_{1}} & =-A^{t} y \\
\overline{r_{2}} & =-A x-u+v+b \\
\overline{r_{3}} & =-U(G+Y) e \\
\overline{r_{4}} & =-V(G-Y) e \\
\overline{D_{u}} & =\left[G+Y+U G_{2}\right] \\
\overline{D_{v}} & =\left[G-Y+V G_{2}\right] \\
\bar{D} & =\left\{-\bar{D}_{u}^{-1} U-\left[\overline{D_{v}}-V U G_{2}^{2} \bar{D}_{u}^{-1}\right]^{-1} V\left(I+\bar{D}_{u}^{-1} U G_{2}\right)^{2}\right\}^{-1}, \\
\bar{r} & =-\left\{{ \overline { r _ { 1 } } } ^ { - 1 } A ^ { t } \overline { D } \left[{\overline{r_{2}}}^{-1} \bar{D}_{u}^{-1} \bar{r}_{3}+\left[\bar{D}_{v}-V U G_{2}^{2} \bar{D}_{u}^{-1}\right]^{-1}\left(I+\bar{D}_{u}^{-1} U G_{2}\right) \times\right.\right. \\
& \left.\times\left({\overline{r_{4}}}^{-1} \bar{D}_{u}^{-1} V G_{2}{\overline{r_{3}}}^{-1}\right]\right\} .
\end{aligned}
$$

Obtemos então as direções afim do nosso problema [18]. Agora, podemos impor um 
valor para o parâmetro barreira $\mu$ e resolver o segundo sistema linear que é dado por

$$
\left[\begin{array}{cccc}
0 & A^{t} & 0 & 0 \\
A & 0 & I & -I \\
0 & U & G+Y+U G_{2} & U G_{2} \\
0 & -V & V G_{2} & G-Y+V G_{2}
\end{array}\right]\left[\begin{array}{c}
\hat{d x} \\
\hat{d y} \\
\hat{d u} \\
\hat{d v}
\end{array}\right]=\left[\begin{array}{c}
\hat{r_{1}} \\
\hat{r_{2}} \\
\hat{r_{3}} \\
\hat{r_{4}}
\end{array}\right]
$$

onde

$$
\begin{aligned}
& \hat{r_{1}}=-A^{t} y, \\
& \hat{r_{2}}=-A x-u+v+b, \\
& \hat{r_{3}}=-U(G+Y) e+\mu e-d \bar{U} d \bar{V} e \quad \mathrm{e} \\
& \hat{r_{4}}=-V(G-Y) e+\mu e-d \bar{U} \overline{d V} e,
\end{aligned}
$$

onde $\bar{d} \bar{U}$ e $d \bar{V}$ são matrizes diagonais de $\overline{d u}$ e $\overline{d v}$ respectivamente (direções afim obtidas no primeiro sistema linear).

Observe que temos a mesma matriz nos dois sistemas lineares, a diferença entre eles se encontra apenas nos vetores $\overline{r_{3}}, \hat{r_{3}}$ e $\overline{r_{4}}, \hat{r_{4}}$. A fatoração da matriz não é afetada por $\mu$, pois o mesmo aparece apenas nos vetores $\hat{r_{3}}$ e $\hat{r_{4}}$.

Assim, temos as direções

$$
\begin{aligned}
\hat{d x} & =\left(A^{t} \bar{D} A\right)^{-1} \hat{r} \\
\hat{d y} & =\bar{D}\left\{\hat{r_{2}}-A \hat{d x}-{\overline{D_{u}}}^{-1} \hat{r_{3}}+\left[\bar{D}_{v}-V U G_{2}^{2}{\overline{D_{u}}}^{-1}\right]^{-1}\left(I+\bar{D}_{u}^{-1} U G_{2}\right)\left(\hat{r_{4}}-{\overline{D_{u}}}^{-1} V G_{2} \hat{r_{3}}\right)\right\}, \\
\hat{d v} & =\left[\bar{D}_{v}-V U G_{2}^{2} \bar{D}_{u}^{-1}\right]^{-1}\left[\hat{r_{4}}+V \hat{d y}+\bar{D}_{u}^{-1}\left(-V G_{2} \hat{r_{3}}+V U G_{2} \hat{d y}\right)\right], \\
\hat{d u} & =\bar{D}_{u}^{-1}\left(\hat{r_{3}}-U \hat{d y}-U G_{2} \hat{d v}\right),
\end{aligned}
$$

onde

$$
\begin{aligned}
& \hat{r_{1}}=-A^{t} y, \\
& \hat{r_{2}}=-A x-u+v+b, \\
& \hat{r_{3}}=-U(G+Y) e+\mu e-d \bar{U} d \bar{V} e,
\end{aligned}
$$




$$
\begin{aligned}
\hat{r_{4}} & =-V(G-Y) e+\mu e-\bar{d} \bar{U} \bar{V} e, \\
\bar{D}_{u} & =\left[G+Y+U G_{2}\right] \\
\bar{D}_{v} & =\left[G-Y+V G_{2}\right] \\
\bar{D} & =\left\{-{\overline{D_{u}}}^{-1} U-\left[\bar{D}_{v}-V U G_{2}^{2}{\overline{D_{u}}}^{-1}\right]^{-1} V\left(I+{\overline{D_{u}}}^{-1} U G_{2}\right)^{2}\right\}^{-1}, \\
\hat{r} & =-\left\{\hat{r}_{1}-A^{t} \bar{D}\left[\hat{r_{2}}-{\overline{D_{u}}}^{-1}{\hat{r_{3}}}^{-1}\left[{\overline{D_{v}}}^{-1} V U G_{2}^{2}{\overline{D_{u}}}^{-1}\right]^{-1}\left(I+{\overline{D_{u}}}^{-1} U G_{2}\right) \times\right.\right. \\
& \left.\left.\times\left(\hat{r_{4}}-{\overline{D_{u}}}^{-1} V G_{2} \hat{r_{3}}\right)\right]\right\} .
\end{aligned}
$$

O passo $\alpha$, onde $u$ e $v$ permaneçam estritamente positivas, é dado por

$$
\alpha=\min \left\{\tau\left(\min _{\hat{d} u_{i}<0}-\frac{u_{i}}{\hat{d} u_{i}}\right), \tau\left(\min _{\hat{d} v_{i}<0}-\frac{v_{i}}{\hat{d} v_{i}}\right), 1\right\},
$$

onde $\tau=0.9995[30]$.

Conhecendo as direções e os passos, todas as variáveis do problema podem ser atualizadas por

$$
\begin{aligned}
& x^{k+1}=x^{k}+\alpha \hat{d x}, \\
& u^{k+1}=u^{k}+\alpha \hat{d u}, \\
& v^{k+1}=v^{k}+\alpha \hat{d v}, \\
& y^{k+1}=y^{k}+\alpha \hat{d y} .
\end{aligned}
$$

A atualização do parâmetro barreira é dada por

$$
\mu^{k+1}=\frac{\mu^{k}}{\beta}, \quad \text { onde } \beta>1 .
$$

Apresentamos agora o resumo do método de pontos interiores preditor-corretor para o problema $L_{p}$.

$\operatorname{Dados} x^{0}, y^{0}$ e $\left(u^{0}, v^{0}\right)>0, \mu^{0}$ e $\beta>1$.

Para $k=0,1,2, \ldots$ faça

$$
G=p\left(u_{i}+v_{i}\right)^{p-1}
$$




$$
\begin{aligned}
& G_{2}=\operatorname{diag}\left(\frac{p(p-1)}{\left(u_{i}+v_{i}\right)^{2-p}}\right) \\
& \overline{r_{1}}=-A^{t} y \\
& \overline{r_{2}}=-A x-u+v+b \\
& \overline{r_{3}}=-U(G+Y) e \\
& \bar{r}_{4}=-V(G-Y) e \\
& \bar{D}_{u}=\left[G+Y+U G_{2}\right] \\
& \bar{D}_{v}=\left[G-Y+V G_{2}\right]
\end{aligned}
$$

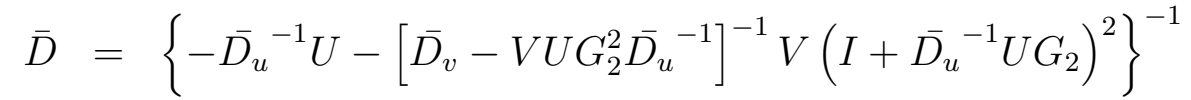

$$
\begin{aligned}
& \bar{r}=-\left\{{ \overline { r _ { 1 } } } _ { - } A ^ { t } \overline { D } \left[{\overline{r_{2}}}^{-\bar{D}_{u}}{ }^{-1}{\overline{r_{3}}}\left[\bar{D}_{v}-V U G_{2}^{2} \bar{D}_{u}^{-1}\right]^{-1}\left(I+\bar{D}_{u}^{-1} U G_{2}\right) \times\right.\right. \\
& \left.\left.\times\left(\overline{r_{4}}-{\overline{D_{u}}}^{-1} V G_{2}{\overline{r_{3}}}\right)\right]\right\} \\
& \overline{d x}=\left(A^{t} \bar{D} A\right)^{-1} \bar{r} \\
& \overline{d y}=\bar{D}\left\{\overline{r_{2}}-A \overline{d x}-{\overline{D_{u}}}^{-1} \overline{r_{3}}+\left[\overline{D_{v}}-V U G_{2}^{2}{\overline{D_{u}}}^{-1}\right]^{-1}\left(I+{\overline{D_{u}}}^{-1} U G_{2}\right)\left(\overline{r_{4}}-{\overline{D_{u}}}^{-1} V G_{2} \overline{r_{3}}\right)\right\} \\
& \overline{d v}=\left[\bar{D}_{v}-V U G_{2}^{2}{\overline{D_{u}}}^{-1}\right]^{-1}\left[\overline{r_{4}}+V \overline{d y}+{\overline{D_{u}}}^{-1}\left(-V G_{2} \overline{r_{3}}+V U G_{2} \overline{d y}\right)\right] \\
& \overline{d u}=\bar{D}_{u}^{-1}\left(\overline{r_{3}}-U \overline{d y}-U G_{2} \overline{d v}\right) \\
& \hat{r_{1}}=-A^{t} y \\
& \hat{r_{2}}=-A x-u+v+b \\
& \hat{r_{3}}=-U(G+Y) e+\mu e-\bar{d} \bar{U} \bar{V} e \\
& \hat{r_{4}}=-V(G-Y) e+\mu e-d \bar{U} d \bar{V} e \\
& \hat{r}=-\left\{\hat{r_{1}}-A^{t} \bar{D}\left[\hat{r_{2}}-{\overline{D_{u}}}^{-1} \hat{r_{3}}+\left[\bar{D}_{v}-V U G_{2}^{2} \bar{D}_{u}^{-1}\right]^{-1}\left(I+{\overline{D_{u}}}^{-1} U G_{2}\right) \times\right.\right. \\
& \left.\left.\times\left(\hat{r_{4}}-{\overline{D_{u}}}^{-1} V G_{2} \hat{r_{3}}\right)\right]\right\} \\
& \hat{d x}=\left(A^{t} \bar{D} A\right)^{-1} \hat{r} \\
& \hat{d y}=\bar{D}\left\{\hat{r_{2}}-A \hat{d x}-{\overline{D_{u}}}^{-1} \hat{r_{3}}+\left[\bar{D}_{v}-V U G_{2}^{2}{\overline{D_{u}}}^{-1}\right]^{-1}\left(I+{\overline{D_{u}}}^{-1} U G_{2}\right)\left(\hat{r_{4}}-{\overline{D_{u}}}^{-1} V G_{2} \hat{r_{3}}\right)\right\} \\
& \hat{d v}=\left[\bar{D}_{v}-V U G_{2}^{2} \bar{D}_{u}^{-1}\right]^{-1}\left[\hat{r}_{4}+V \hat{d y}+\bar{D}_{u}^{-1}\left(-V G_{2} \hat{r}_{3}+V U G_{2} \hat{d y}\right)\right] \\
& \hat{d u}=\bar{D}_{u}^{-1}\left(\hat{r_{3}}-U \hat{d y}-U G_{2} \hat{d v}\right) \\
& \alpha=\min \left\{\tau\left(\min _{\hat{d} u_{i}<0}-\frac{u_{i}}{\hat{d} u_{i}}\right), \tau\left(\min _{\hat{d}_{i}<0}-\frac{v_{i}}{\hat{d} v_{i}}\right), 1\right\} \\
& u^{k+1}=u^{k}+\alpha \hat{d u}
\end{aligned}
$$




$$
\begin{aligned}
v^{k+1} & =v^{k}+\alpha \hat{d v} \\
x^{k+1} & =x^{k}+\alpha \hat{d x} \\
y^{k+1} & =y^{k}+\alpha \hat{d y} \\
\mu^{k+1} & =\frac{\mu^{k}}{\beta}
\end{aligned}
$$

\subsubsection{Algumas Considerações}

O critério de convergência e o ponto inicial são os mesmos utilizados no método de pontos interiores barreira logarítmica.

Sabemos que na maioria dos métodos de pontos interiores o passo mais caro em termos computacionais é a solução do sistema linear com uma matriz do tipo $A^{t} D A$, onde $D$ é uma matriz diagonal. Apesar de resolvermos dois sistemas lineares em cada iteração, os cálculos utilizados para construir a matriz e sua decomposição são efetuados uma única vez pois a matriz é a mesma nos dois sistemas lineares. Assim, esperamos obter uma redução do esforço computacional em relação ao método de pontos interiores barreira logarítmica como acontece em otimização linear [16].

\subsection{Método Primal-Dual Barreira Logarítmica}

De (4.2), sabemos que

$$
\underbrace{\nabla L}_{J(x, y, u, v)}=\left[\begin{array}{c}
A^{t} y \\
A x+u-v-b \\
\left(G-\mu U^{-1}+Y\right) e \\
\left(G-\mu V^{-1}-Y\right) e
\end{array}\right]=\left[\begin{array}{l}
0 \\
0 \\
0 \\
0
\end{array}\right] .
$$

onde $U$ e $V$ são matrizes diagonais cujos elementos diagonais são $u$ e $v$ respectivamente e $e=(1,1, \ldots, 1)$

Agora, sejam $z_{u}=\mu U^{-1}$ e e $z_{v}=\mu V^{-1} e$. Obtemos assim um problema estritamente 
primal-dual [7],

$$
\underbrace{\nabla L}_{J\left(x, y, u, v, z_{u}, z_{v}\right)}=\left[\begin{array}{c}
A^{t} y \\
A x+u-v-b \\
\left(G-Z_{u}+Y\right) e \\
\left(G-Z_{v}-Y\right) e \\
U Z_{u} e \\
V Z_{v} e
\end{array}\right]=\left[\begin{array}{c}
0 \\
0 \\
0 \\
0 \\
\mu e \\
\mu e
\end{array}\right] .
$$

Utilizando o Método de Newton, chegamos a

$$
\left[\begin{array}{cccccc}
0 & A^{t} & 0 & 0 & 0 & 0 \\
A & 0 & I & -I & 0 & 0 \\
0 & I & G_{2} & G_{2} & -I & 0 \\
0 & -I & G_{2} & G_{2} & 0 & -I \\
0 & 0 & Z_{u} & 0 & U & 0 \\
0 & 0 & 0 & Z_{v} & 0 & V
\end{array}\right]\left[\begin{array}{c}
d x \\
d y \\
d u \\
d v \\
d z_{u} \\
d z_{v}
\end{array}\right]=\left[\begin{array}{c}
r_{1} \\
r_{2} \\
r_{3} \\
r_{4} \\
r_{5} \\
r_{6}
\end{array}\right]
$$

onde

$$
\begin{aligned}
& r_{1}=-A^{t} y, \\
& r_{2}=-A x-u+v+b, \\
& r_{3}=\left(-G+Z_{u}-Y\right) e, \\
& r_{4}=\left(-G+Z_{v}+Y\right) e, \\
& r_{5}=-U Z_{u} e+\mu e \mathrm{e} \\
& r_{6}=-V Z_{v} e+\mu e .
\end{aligned}
$$

Resolvendo o sistema (4.15), obtemos as direções $d x, d y, d u, d v, d z_{u}, d z_{v}$. Assim, temos o sistema

$$
\begin{aligned}
A^{t} d y & =r_{1} \\
A d x+d u-d v & =r_{2}
\end{aligned}
$$




$$
\begin{aligned}
d y+G_{2} d u+G_{2} d v-d z_{u} & =r_{3} \\
-d y+G_{2} d u+G_{2} d v-d z_{v} & =r_{4} \\
Z_{u} d u+U d z_{u} & =r_{5} \\
Z_{v} d v+V d z_{v} & =r_{6} .
\end{aligned}
$$

Da Equação (4.20),

$$
\begin{gathered}
Z_{u} d u+U d z_{u}=r_{5} \\
\Rightarrow U d z_{u}=r_{5}-Z_{u} d u \\
\Rightarrow d z_{u}=U^{-1}\left(r_{5}-Z_{u} d u\right)
\end{gathered}
$$

Da Equação (4.21),

$$
\begin{gathered}
Z_{v} d v+V d z_{v}=r_{6} \\
\Rightarrow V d z_{v}=r_{6}-Z_{v} d v \\
\Rightarrow d z_{v}=V^{-1}\left(r_{6}-Z_{v} d v\right)
\end{gathered}
$$

Da Equação (4.18),

$$
\begin{gathered}
d y+G_{2} d u+G_{2} d v-d z_{u}=r_{3} \\
\Rightarrow G_{2} d u=r_{3}-d y-G_{2} d v+d z_{u} \\
\Rightarrow G_{2} d u=r_{3}-d y-G_{2} d v+U^{-1}\left(r_{5}-Z_{u} d u\right) \\
\Rightarrow\left(G_{2}+U^{-1} Z_{u}\right) d u=r_{3}-d y-G_{2} d v+U^{-1} r_{5} \\
\Rightarrow d u=\left(G_{2}+U^{-1} Z_{u}\right)^{-1}\left(r_{3}-d y-G_{2} d v+U^{-1} r_{5}\right) .
\end{gathered}
$$

Fazendo $D z_{u}=\left(G_{2}+U^{-1} Z_{u}\right)$, obtemos

$$
d u=D z_{u}^{-1}\left(r_{3}-d y-G_{2} d v+U^{-1} r_{5}\right) .
$$


Agora, da Equação (4.19),

$$
\begin{gathered}
-d y+G_{2} d u+G_{2} d v-d z_{v}=r_{4} \\
\Rightarrow G_{2} d v=r_{4}+d y-G_{2} d u+d z_{v} \\
\Rightarrow G_{2} d v=r_{4}+d y-G_{2}\left[D z_{u}^{-1}\left(r_{3}-d y-G_{2} d v+U^{-1} r_{5}\right)\right]+V^{-1}\left(r_{6}-Z_{v} d v\right) \\
\Rightarrow\left(G_{2}+V^{-1} Z_{v}-G_{2}^{2} D z_{u}^{-1}\right) d v=r_{4}+d y+D z_{u}^{-1}\left(-G_{2} r_{3}+G_{2} d y-G_{2} U^{-1} r_{5}\right)+V^{-1} r_{6} \\
\Rightarrow d v=\left(G_{2}+V^{-1} Z_{v}-G_{2}^{2} D z_{u}^{-1}\right)^{-1}\left[r_{4}+d y+D z_{u}^{-1}\left(-G_{2} r_{3}+G_{2} d y-G_{2} U^{-1} r_{5}\right)+V^{-1} r_{6}\right] .
\end{gathered}
$$

Fazendo $D z_{v}=\left(G_{2}+V^{-1} Z_{v}\right)$, obtemos

$$
d v=\left[D z_{v}-G_{2}^{2} D z_{u}^{-1}\right]^{-1}\left[r_{4}+d y+D z_{u}^{-1}\left(-G_{2} r_{3}+G_{2} d y-G_{2} U^{-1} r_{5}\right)+V^{-1} r_{6}\right] .
$$

Da Equação (4.17),

$$
A d x+d u-d v=r_{2}
$$

$$
\begin{array}{ll}
\Rightarrow \quad & A d x+D z_{u}^{-1}\left(r_{3}-d y-G_{2} d v+U^{-1} r_{5}\right)-\left[D z_{v}-G_{2}^{2} D z_{u}^{-1}\right]^{-1}\left[r_{4}+d y+D z_{u}^{-1}\left(-G_{2} r_{3}+\right.\right. \\
+ & \left.\left.G_{2} d y-G_{2} U^{-1} r_{5}\right)+V^{-1} r_{6}\right]=r_{2} \\
\Rightarrow & \quad A d x+D z_{u}^{-1}\left\{r_{3}-d y-G_{2}\left[D z_{v}-G_{2}^{2} D z_{u}^{-1}\right]^{-1}\left[r_{4}+d y+D z_{u}^{-1}\left(-G_{2} r_{3}+G_{2} d y-G_{2} U^{-1} r_{5}\right)+\right.\right. \\
& \left.\left.+\quad V^{-1} r_{6}\right]+U^{-1} r_{5}\right\}-\left[D z_{v}-G_{2}^{2} D z_{u}^{-1}\right]^{-1}\left[r_{4}+d y+D z_{u}^{-1}\left(-G_{2} r_{3}+G_{2} d y-G_{2} U^{-1} r_{5}\right)+\right. \\
+ & \left.V^{-1} r_{6}\right]=r_{2} \\
\Rightarrow \quad & -D_{u}^{-1} d y-D z_{u}^{-1} G_{2}\left[D z_{v}-G_{2}^{2} D z_{u}^{-1}\right]^{-1}\left(I+D z_{u}^{-1} G_{2}\right) d y-\left[D z_{v}-G_{2}^{2} D z_{u}^{-1}\right]^{-1} \times \\
\times & \left(I+D z_{u}^{-1} G_{2}\right) d y=r_{2}-A d x-D z_{u}^{-1}\left\{r_{3}-G_{2}\left[( D z _ { v } - G _ { 2 } ^ { 2 } D z _ { u } ^ { - 1 } ) ^ { - 1 } \left(r_{4}+D z_{u}^{-1} \times\right.\right.\right. \\
\times & \left.\left.\left.\quad\left(-G_{2} r_{3}-G_{2} U^{-1} r_{5}\right)+V^{-1} r_{6}\right)\right]+U^{-1} r_{5}\right\}-\left(D z_{v}-G_{2}^{2} D z_{u}^{-1}\right)^{-1}\left(r_{4}+D z_{u}^{-1} \times\right. \\
\times & \left.\left(-G_{2} r_{3}-G_{2} U^{-1} r_{5}\right)+V^{-1} r_{6}\right) \\
& \quad\left\{-D z_{u}^{-1}-\left[D z_{v}-G_{2}^{2} D z_{u}^{-1}\right]^{-1}\left(I+D z_{u}^{-1} G_{2}\right)^{2}\right\} d y=r_{2}-A d x-D z_{u}^{-1} r_{3}+ \\
\Rightarrow \quad & \quad\left[D z_{v}-G_{2}^{2} D z_{u}^{-1}\right]^{-1}\left(I+D z_{u}^{-1} G_{2}\right)\left[r_{4}-D z_{u}^{-1}\left(G_{2} r_{3}-G_{2} U^{-1} r_{5}\right)+V^{-1} r_{6}\right]
\end{array}
$$




$$
\begin{aligned}
\Rightarrow d y & =\left\{-D z_{u}^{-1}-\left[D z_{v}-G_{2}^{2} D z_{u}^{-1}\right]^{-1}\left(I+D z_{u}^{-1} G_{2}\right)^{2}\right\}^{-1}\left\{r_{2}-A d x-D z_{u}^{-1} r_{3}+\right. \\
& \left.+\left[D z_{v}-G_{2}^{2} D z_{u}^{-1}\right]^{-1}\left(I+D z_{u}^{-1} G_{2}\right)\left[r_{4}-D z_{u}^{-1}\left(G_{2} r_{3}-G_{2} U^{-1} r_{5}\right)+V^{-1} r_{6}\right]\right\} .
\end{aligned}
$$

Fazendo $D=\left\{-D z_{u}^{-1}-\left[D z_{v}-G_{2}^{2} D z_{u}^{-1}\right]^{-1}\left(I+D z_{u}^{-1} G_{2}\right)^{2}\right\}^{-1}$, temos

$$
\begin{aligned}
d y & =D\left\{r_{2}-A d x-D z_{u}^{-1} r_{3}+\left[D z_{v}-G_{2}^{2} D z_{u}^{-1}\right]^{-1}\left(I+D z_{u}^{-1} G_{2}\right)\left[r_{4}-D z_{u}^{-1} \times\right.\right. \\
& \left.\left.\times\left(G_{2} r_{3}-G_{2} U^{-1} r_{5}\right)+V^{-1} r_{6}\right]\right\} .
\end{aligned}
$$

Finalmente, de (4.16), obtemos

$$
\begin{aligned}
& A^{t} d y=r_{1} \\
\Rightarrow & \quad A^{t} D\left\{r_{2}-A d x-D z_{u}^{-1} r_{3}+\left[D z_{v}-G_{2}^{2} D z_{u}^{-1}\right]^{-1}\left(I+D z_{u}^{-1} G_{2}\right)\left[r_{4}-D z_{u}^{-1} \times\right.\right. \\
\times & \left.\left.\left(-G_{2} r_{3}-G_{2} U^{-1} r_{5}\right)+V^{-1} r_{6}\right]\right\}=r_{1} \\
\Rightarrow & -\quad A^{t} D A d x=r_{1}-A^{t} D\left\{r_{2}-D z_{u}^{-1} r_{3}+\left[D z_{v}-G_{2}^{2} D z_{u}^{-1}\right]^{-1}\left(I+D z_{u}^{-1} G_{2}\right) \times\right. \\
\times & {\left.\left[r_{4}-D z_{u}^{-1}\left(-G_{2} r_{3}-G_{2} U^{-1} r_{5}\right)+V^{-1} r_{6}\right]\right\} } \\
\Rightarrow d x= & \left(-A^{t} D A\right)^{-1}\left\{r_{1}-A^{t} D\left[r_{2}-D z_{u}^{-1} r_{3}+\left[D z_{v}-G_{2}^{2} D z_{u}^{-1}\right]^{-1}\left(I+D z_{u}^{-1} G_{2}\right) \times\right.\right. \\
\times & {\left.\left.\left[r_{4}-D z_{u}^{-1}\left(-G_{2} r_{3}-G_{2} U^{-1} r_{5}\right)+V^{-1} r_{6}\right]\right]\right\} . }
\end{aligned}
$$

Definindo $r=-\left\{r_{1}-A^{t} D\left[r_{2}-D z_{u}^{-1} r_{3}+\left[D z_{v}-G_{2}^{2} D z_{u}^{-1}\right]^{-1}\left(I+D z_{u}^{-1} G_{2}\right) \times\right.\right.$ $\left.\left.\times\left[r_{4}-D z_{u}^{-1}\left(-G_{2} r_{3}-G_{2} U^{-1} r_{5}\right)+V^{-1} r_{6}\right]\right]\right\}$, obtemos

$$
d x=\left(A^{t} D A\right)^{-1} r .
$$

Agora, vamos calcular o passo $\alpha$, onde $u, v, d z_{u}$ e $d z_{v}$ permaneçam estritamente positivas.

$$
\alpha=\min \left\{\tau\left(\min _{d u_{i}<0}-\frac{u_{i}}{d u_{i}}\right), \tau\left(\min _{d v_{i}<0}-\frac{v_{i}}{d v_{i}}\right), \tau\left(\min _{d z_{u i}<0}-\frac{z_{u i}}{d z_{u i}}\right), \tau\left(\min _{d z_{v i}<0}-\frac{z_{v i}}{d z_{v i}}\right), 1\right\},
$$

onde $\tau=0.9995[30]$. 
Conhecendo as direções e os passos, todas as variáveis do problema podem ser atualizadas por

$$
\begin{gathered}
x^{k+1}=x^{k}+\alpha d x, \\
y^{k+1}=y^{k}+\alpha d y, \\
u^{k+1}=u^{k}+\alpha d u, \\
v^{k+1}=v^{k}+\alpha d v, \\
z_{u}^{k+1}=z_{u}^{k}+\alpha d z_{u}, \\
z_{v}^{k+1}=z_{v}^{k}+\alpha d z_{v} .
\end{gathered}
$$

A atualização do parâmetro barreira é dada por

$$
\mu=\gamma \phi, \quad \text { onde } \gamma=u^{t} z_{u}+v^{t} z_{v} \text { e } \phi=\frac{1}{m \sqrt{m}} .
$$

Apresentamos agora o resumo do método de pontos interiores primal-dual barreira logarítmica para o problema $L_{p}$.

$\operatorname{Dados} x^{0}, y^{0}$ e $\left(u^{0}, v^{0}\right)>0,\left(z_{u}^{0}, z_{v}^{0}\right)>0, \mu^{0}$ e $\beta>1$.

Para $k=0,1,2, \ldots$ faça

$$
\begin{aligned}
G & =p\left(u_{i}+v_{i}\right)^{p-1} \\
G_{2} & =\operatorname{diag}\left(\frac{p(p-1)}{\left(u_{i}+v_{i}\right)^{2-p}}\right) \\
r_{1} & =-A^{t} y \\
r_{2} & =-A x-u+v+b \\
r_{3} & =\left(-G+Z_{u}-Y\right) e \\
r_{4} & =\left(-G+Z_{v}+Y\right) e \\
r_{5} & =-U Z_{u} e+\mu e \\
r_{6} & =-V Z_{v} e+\mu e \\
D z_{u} & =\left(G U_{2}+U^{-1} Z_{u}\right)
\end{aligned}
$$




$$
\begin{aligned}
& D z_{v}=\left(G_{2}+V^{-1} Z_{v}\right) \\
& D=\left\{-D z_{u}^{-1}-\left[D z_{v}-G_{2}^{2} D z_{u}^{-1}\right]^{-1}\left(I+D z_{u}^{-1} G_{2}\right)^{2}\right\}^{-1} \\
& r=-\left\{r_{1}-A^{t} D\left[r_{2}-D z_{u}^{-1} r_{3}+\left[D z_{v}-G_{2}^{2} D z_{u}^{-1}\right]^{-1}\left(I+D z_{u}^{-1} G_{2}\right) \times\right.\right. \\
& \left.\left.\times\left[r_{4}-D z_{u}^{-1}\left(-G_{2} r_{3}-G_{2} U^{-1} r_{5}\right)+V^{-1} r_{6}\right]\right]\right\} \\
& d x=\left(A^{t} D A\right)^{-1} r \\
& d y=D\left\{r_{2}-A d x-D z_{u}^{-1} r_{3}+\left[D z_{v}-G_{2}^{2} D z_{u}^{-1}\right]^{-1}\left(I+D z_{u}^{-1} G_{2}\right)\left[r_{4}-D z_{u}^{-1} \times\right.\right. \\
& \left.\left.\times \quad\left(G_{2} r_{3}-G_{2} U^{-1} r_{5}\right)+V^{-1} r_{6}\right]\right\} \\
& d v=\left[D z_{v}-G_{2}^{2} D z_{u}^{-1}\right]^{-1}\left[r_{4}+d y+D z_{u}^{-1}\left(-G_{2} r_{3}+G_{2} d y-G_{2} U^{-1} r_{5}\right)+V^{-1} r_{6}\right] \\
& d u=D z_{u}^{-1}\left(r_{3}-d y-G_{2} d v+U^{-1} r_{5}\right) \\
& d z_{u}=U^{-1}\left(r_{5}-Z_{u} d u\right) \\
& d z_{v}=V^{-1}\left(r_{6}-Z_{v} d v\right) \\
& \alpha=\min \left\{\tau\left(\min _{d u_{i}<0}-\frac{u_{i}}{d u_{i}}\right), \tau\left(\min _{d v_{i}<0}-\frac{v_{i}}{d v_{i}}\right), \tau\left(\min _{d z_{u i}<0}-\frac{z_{u i}}{d z_{u i}}\right), \tau\left(\min _{d z_{v i}<0}-\frac{z_{v i}}{d z_{v i}}\right), 1\right\} \\
& u^{k+1}=u^{k}+\alpha d u \\
& v^{k+1}=v^{k}+\alpha d v \\
& x^{k+1}=x^{k}+\alpha d x \\
& y^{k+1}=y^{k}+\alpha d y \\
& z_{u}^{k+1}=z_{u}^{k}+\alpha d z_{u} \\
& z_{v}^{k+1}=z_{v}^{k}+\alpha d z_{v} \\
& \gamma=u^{t} z_{u}+v^{t} z_{v} \\
& \phi=\frac{1}{m \sqrt{m}} \\
& \mu=\gamma \phi
\end{aligned}
$$

Até convergir.

\subsubsection{Critério de Convergência}

O critério de convergência é baseado nas condições de otimalidade (4.14):

$$
N=\frac{\|\nabla L\|}{\left(1+\|x\|+\|u\|+\|v\|+\|y\|+\left\|z_{u}\right\|+\left\|z_{v}\right\|\right)(2 n)} \leq \epsilon .
$$


Utilizamos também um critério de convergência baseado na diferença dos valores de $N$ atual e da iteração anterior:

$$
\left|N^{k+1}-N^{k}\right| \leq \epsilon_{1}
$$

\subsubsection{Algumas Considerações}

Note que apesar de contar mais variáveis, o maior esforço computacional deste método consiste em resolver um sistema linear com a mesma dimensão e estrutura do método barreira logarítmica. Um ponto inicial para as novas variáveis pode ser dado por (4.1.2) $\mathrm{e} z_{u}=z_{v}=(1, \ldots, 1)^{t}$.

\subsection{Método Primal-Dual Preditor-Corretor}

Sabemos que no método primal-dual preditor-corretor, primeiramente tomamos a direção afim em que o parâmetro barreira $\mu=0$. Então, temos que resolver o sistema

$$
\left[\begin{array}{cccccc}
0 & A^{t} & 0 & 0 & 0 & 0 \\
A & 0 & I & -I & 0 & 0 \\
0 & I & G_{2} & G_{2} & -I & 0 \\
0 & -I & G_{2} & G_{2} & 0 & -I \\
0 & 0 & Z_{u} & 0 & U & 0 \\
0 & 0 & 0 & Z_{v} & 0 & V
\end{array}\right]\left[\begin{array}{c}
\overline{d x} \\
\overline{d y} \\
\overline{d u} \\
\overline{d v} \\
\overline{d z_{u}} \\
\overline{d z_{v}}
\end{array}\right]=\left[\begin{array}{c}
\overline{r_{1}} \\
\overline{r_{2}} \\
\overline{r_{3}} \\
\overline{r_{4}} \\
\overline{r_{5}} \\
\overline{r_{6}}
\end{array}\right]
$$

onde

$$
\begin{aligned}
& \overline{r_{1}}=-A^{t} y, \\
& \overline{r_{2}}=-A x-u+v+b, \\
& \overline{r_{3}}=\left(-G+Z_{u}-Y\right) e, \\
& \overline{r_{4}}=\left(-G+Z_{v}+Y\right) e, \\
& \overline{r_{5}}=-U Z_{u} e \mathrm{e} \\
& \overline{r_{6}}=-V Z_{v} e .
\end{aligned}
$$


que é o mesmo sistema dado pela Equação (4.15) com $\mu=0$.

Resolvendo o sistema (4.22), obtemos as direções $\overline{d x}, \overline{d y}, \overline{d u}, \overline{d v}, \bar{z}_{u}, \bar{d} \bar{z}_{v}$. Assim, temos o sistema

$$
\begin{aligned}
& A^{t} \overline{d y}=\overline{r_{1}} \\
& A \overline{d x}+\overline{d u}-\overline{d v}=\overline{r_{2}} \\
& \overline{d y}+G_{2} \overline{d u}+G_{2} \overline{d v}-\overline{d z_{u}}=\overline{r_{3}} \\
&-\overline{d y}+G_{2} \overline{d u}+G_{2} \overline{d v}-\overline{d z_{v}}=\overline{r_{4}} \\
& Z_{u} \overline{d u}+U \bar{d}_{u}=\overline{r_{5}} \\
& Z_{v} \overline{d v}+V \overline{d z_{v}}=\bar{r}_{6} .
\end{aligned}
$$

Fazendo a mesma eliminação de variáveis utilizada no método primal-dual barreira logarítmica, obtemos as direções

$$
\begin{aligned}
& \overline{d x}=\left(A^{t} \bar{D} A\right)^{-1} \bar{r} \\
& \overline{d y}=\bar{D}\left\{{\overline{r_{2}}}_{-A \overline{d x}}-{\overline{D z_{u}}}^{-1}{\overline{r_{3}}}+\left[{\overline{D z_{v}}}-G_{2}^{2} \overline{D z}_{u}{ }^{-1}\right]^{-1}\left(I+\overline{D z}_{u}{ }^{-1} G_{2}\right)\left[{\overline{r_{4}}}-\overline{D z}_{u}{ }^{-1} \times\right.\right. \\
& \left.\left.\times\left(G_{2} \overline{r_{3}}-G_{2} U^{-1} \overline{r_{5}}\right)+V^{-1} \overline{r_{6}}\right]\right\}, \\
& \overline{d v}=\left[\overline{D z}_{v}-G_{2}^{2}{\overline{D z_{u}}}^{-1}\right]^{-1}\left[{\overline{r_{4}}}+\overline{d y}+\overline{D z}_{u}^{-1}\left(-G_{2}{\overline{r_{3}}}^{-G_{2}} \overline{d y}-G_{2} U^{-1} \bar{r}_{5}\right)+V^{-1} \overline{r_{6}}\right] \\
& \overline{d u}=\overline{D z}_{u}^{-1}\left(\overline{r_{3}}-\overline{d y}-G_{2} \overline{d v}+U^{-1} \overline{r_{5}}\right) \text {, } \\
& \bar{d}_{u}=U^{-1}\left(\bar{r}_{5}-Z_{u} \overline{d u}\right), \\
& \overline{d z}_{v}=V^{-1}\left(\bar{r}_{6}-Z_{v} \overline{d v}\right)
\end{aligned}
$$

onde

$$
\begin{aligned}
& \overline{r_{1}}=-A^{t} y, \\
& \overline{r_{2}}=-A x-u+v+b, \\
& \overline{r_{3}}=\left(-G+Z_{u}-Y\right) e, \\
& \overline{r_{4}}=\left(-G+Z_{v}+Y\right) e, \\
& \overline{r_{5}}=-U Z_{u} e, \\
& \overline{r_{6}}=-V Z_{v} e,
\end{aligned}
$$




$$
\begin{aligned}
& \overline{D z_{u}}=\left(G_{2}+U^{-1} Z_{u}\right), \\
& \overline{D z_{v}}=\left(G_{2}+V^{-1} Z_{v}\right) \text {, } \\
& \bar{D}=\left\{-\overline{D z}_{u}^{-1}-\left[\overline{D z}_{v}-G_{2}^{2} \overline{D z}_{u}^{-1}\right]^{-1}\left(I+\overline{D z}_{u}^{-1} G_{2}\right)^{2}\right\}^{-1}, \\
& \bar{r}=-\left\{\overline{r_{1}}-A^{t} \bar{D}\left[{\overline{r_{2}}}_{-\overline{D z_{u}}}{ }^{-1}{\overline{r_{3}}}+\left[\overline{D z_{v}}-G_{2}^{2}{\overline{D z_{u}}}^{-1}\right]^{-1}\left(I+{\overline{D z_{u}}}^{-1} G_{2}\right) \times\right.\right. \\
& \left.\left.\times\left[{\overline{r_{4}}}^{-}{\overline{D z_{u}}}^{-1}\left(-G_{2}{\overline{r_{3}}}_{-} G_{2} U^{-1}{\overline{r_{5}}}\right)+V^{-1} \overline{r_{6}}\right]\right]\right\} \text {. }
\end{aligned}
$$

Obtemos então as direções afim do nosso problema. Agora, podemos impor um valor para o parâmetro barreira $\mu$ e resolver o segundo sistema linear que é dado por

$$
\left[\begin{array}{cccccc}
0 & A^{t} & 0 & 0 & 0 & 0 \\
A & 0 & I & -I & 0 & 0 \\
0 & I & G_{2} & G_{2} & -I & 0 \\
0 & -I & G_{2} & G_{2} & 0 & -I \\
0 & 0 & Z_{u} & 0 & U & 0 \\
0 & 0 & 0 & Z_{v} & 0 & V
\end{array}\right]\left[\begin{array}{c}
\hat{d x} \\
\hat{d y} \\
\hat{d u} \\
\hat{d v} \\
\hat{d z_{u}} \\
\hat{d z_{v}}
\end{array}\right]=\left[\begin{array}{c}
\hat{r_{1}} \\
\hat{r_{2}} \\
\hat{r_{3}} \\
\hat{r_{4}} \\
\hat{r_{5}} \\
\hat{r_{6}}
\end{array}\right]
$$

onde

$$
\begin{aligned}
& \hat{r_{1}}=-A^{t} y, \\
& \hat{r_{2}}=-A x-u+v+b, \\
& \hat{r_{3}}=\left(-G+Z_{u}-Y\right) e, \\
& \hat{r_{4}}=\left(-G+Z_{v}+Y\right) e, \\
& \hat{r_{5}}=-U Z_{u} e+\mu e-d \bar{U} d \bar{Z}_{u} e \mathrm{e}, \\
& \hat{r_{6}}=-V Z_{v} e+\mu e-d \bar{V} d \bar{Z}_{v} e,
\end{aligned}
$$

onde $d \bar{U}, d \bar{V}, d \bar{Z}_{u}$ e $d \bar{Z}_{v}$ são matrizes diagonais de $\overline{d u}, \overline{d v}, d \bar{z}_{u}$ e $d \bar{z}_{v}$ respectivamente (direções afins obtidas no primeiro sistema linear).

Lembremos novamente que a matriz é mesma nos dois sistemas lineares, a diferença entre eles se encontra apenas nos vetores $\overline{r_{3}}, \hat{r_{3}}$ e $\overline{r_{4}}, \hat{r_{4}}$. A fatoração da matriz não é 
afetada por $\mu$, pois o mesmo aparece apenas nos vetores $\hat{r_{3}}$ e $\hat{r_{4}}$.

Assim, temos as direções

$$
\begin{aligned}
& \hat{d x}=\left(A^{t} \bar{D} A\right)^{-1} \hat{r} \\
& \hat{d y}=\bar{D}\left\{\hat{r}_{2}-A \hat{d x}-\overline{D z}_{u}^{-1} \hat{r}_{3}+\left[\overline{D z}_{v}-G_{2}^{2} \overline{D z}_{u}^{-1}\right]^{-1}\left(I+\overline{D z}_{u}^{-1} G_{2}\right)\left[{\hat{r_{4}}}^{-D z_{u}}{ }^{-1} \times\right.\right. \\
& \left.\left.\times\left(G_{2} \hat{r_{3}}-G_{2} U^{-1} \hat{r_{5}}\right)+V^{-1} \hat{r_{6}}\right]\right\}, \\
& \hat{d v}=\left[\overline{D z}_{v}-G_{2}^{2} \overline{D z}_{u}^{-1}\right]^{-1}\left[\hat{r_{4}}+\hat{d y}+\overline{D z}_{u}^{-1}\left(-G_{2} \hat{r_{3}}+G_{2} \hat{d y}-G_{2} U^{-1} \hat{r_{5}}\right)+V^{-1} \hat{r_{6}}\right] \\
& \hat{d u}=\overline{D z}_{u}^{-1}\left(\hat{r_{3}}-\hat{d y}-G_{2} \hat{d v}+U^{-1} \hat{r_{5}}\right) \text {, } \\
& \hat{d z_{u}}=U^{-1}\left(\hat{r}_{5}-Z_{u} \hat{d u}\right), \\
& \hat{d z_{v}}=V^{-1}\left(\hat{r}_{6}-Z_{v} \hat{d v}\right)
\end{aligned}
$$

onde

$$
\begin{aligned}
& \hat{r_{1}}=-A^{t} y \\
& \hat{r_{2}}=-A x-u+v+b \text {, } \\
& \hat{r_{3}}=\left(-G+Z_{u}-Y\right) e, \\
& \hat{r_{4}}=\left(-G+Z_{v}+Y\right) e, \\
& \hat{r_{5}}=-U Z_{u} e \\
& \hat{r_{6}}=-V Z_{v} e \\
& \overline{D z_{u}}=\left(G_{2}+U^{-1} Z_{u}\right) \\
& \overline{D z_{v}}=\left(G_{2}+V^{-1} Z_{v}\right) \\
& \bar{D}=\left\{-\overline{D z}_{u}^{-1}-\left[\overline{D z}_{v}-G_{2}^{2} \overline{D z_{u}}{ }^{-1}\right]^{-1}\left(I+\overline{D z}_{u}^{-1} G_{2}\right)^{2}\right\}^{-1}, \\
& \hat{r}=-\left\{\hat{r_{1}}-A^{t} \bar{D}\left[{\hat{r_{2}}}^{-D_{z_{u}}}{ }^{-1}{\hat{r_{3}}}+\left[{\overline{D z_{v}}}^{-} G_{2}^{2}{\overline{D z_{u}}}^{-1}\right]^{-1}\left(I+\overline{D z}_{u}^{-1} G_{2}\right) \times\right.\right. \\
& \left.\left.\times\left[{\hat{r_{4}}}^{-}-\overline{D z}_{u}^{-1}\left(-G_{2} \hat{r_{3}}-G_{2} U^{-1} \hat{r}_{5}\right)+V^{-1} \hat{r_{6}}\right]\right]\right\} .
\end{aligned}
$$

Agora, vamos calcular o passo $\alpha$, onde $u, v, d z_{u}$ e $d z_{v}$ permaneçam estritamente positivas.

$$
\alpha=\min \left\{\tau\left(\min _{d u_{i}<0}-\frac{u_{i}}{d u_{i}}\right), \tau\left(\min _{d v_{i}<0}-\frac{v_{i}}{d v_{i}}\right), \tau\left(\min _{d z_{u i}<0}-\frac{z_{u i}}{d z_{u i}}\right), \tau\left(\min _{d z_{v i}<0}-\frac{z_{v i}}{d z_{v i}}\right), 1\right\},
$$


onde $\tau=0.9995[30]$.

Conhecendo as direções e os passos, todas as variáveis do problema podem ser atualizadas por

$$
\begin{aligned}
& x^{k+1}=x^{k}+\alpha d x, \\
& y^{k+1}=y^{k}+\alpha d y, \\
& u^{k+1}=u^{k}+\alpha d u, \\
& v^{k+1}=v^{k}+\alpha d v, \\
& z_{u}^{k+1}=z_{u}^{k}+\alpha d z_{u}, \\
& z_{v}^{k+1}=z_{v}^{k}+\alpha d z_{v} .
\end{aligned}
$$

A atualização do parâmetro barreira é dada por

$$
\mu=\gamma \phi, \quad \text { onde } \gamma=u^{t} z_{u}+v^{t} z_{v} \text { e } \phi=\frac{1}{m \sqrt{m}}
$$

Apresentamos agora o resumo do método de pontos interiores primal-dual preditorcorretor para o problema $L_{p}$.

Dados $x^{0}, y^{0}$ e $\left(u^{0}, v^{0}\right)>0,\left(z_{u}^{0}, z_{v}^{0}\right)>0, \mu^{0}$ e $\beta>1$.

Para $k=0,1,2, \ldots$ faça

$$
\begin{aligned}
G & =p\left(u_{i}+v_{i}\right)^{p-1} \\
G_{2} & =\operatorname{diag}\left(\frac{p(p-1)}{\left(u_{i}+v_{i}\right)^{2-p}}\right) \\
\overline{r_{1}} & =-A^{t} y \\
\overline{r_{2}} & =-A x-u+v+b \\
\overline{r_{3}} & =\left(-G+Z_{u}-Y\right) e \\
\overline{r_{4}} & =\left(-G+Z_{v}+Y\right) e \\
\overline{r_{5}} & =-U Z_{u} e
\end{aligned}
$$




$$
\begin{aligned}
& \overline{r_{6}}=-V Z_{v} e \\
& \overline{D z_{u}}=\left(G_{2}+U^{-1} Z_{u}\right) \\
& \overline{D z_{v}}=\left(G_{2}+V^{-1} Z_{v}\right) \\
& \bar{D}=\left\{-\overline{D z}_{u}^{-1}-\left[\overline{D z}_{v}-G_{2}^{2} \overline{D z}_{u}^{-1}\right]^{-1}\left(I+\overline{D z}_{u}^{-1} G_{2}\right)^{2}\right\}^{-1} \\
& \bar{r}=-\left\{\overline{r_{1}}-A^{t} \bar{D}\left[{\overline{r_{2}}}_{-\overline{D z_{u}}}^{-1} \overline{r_{3}}+\left[\overline{D z_{v}}-G_{2}^{2} \overline{D z}_{u}{ }^{-1}\right]^{-1}\left(I+\overline{D z}_{u}{ }^{-1} G_{2}\right) \times\right.\right.
\end{aligned}
$$

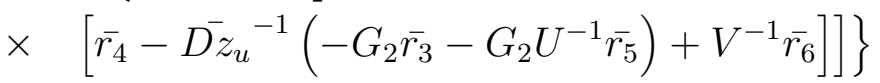

$$
\begin{aligned}
& \overline{d x}=\left(A^{t} \bar{D} A\right)^{-1} \bar{r} \\
& \overline{d y}=\bar{D}\left\{{\overline{r_{2}}}-A \overline{d x}-\overline{D z}_{u}{ }^{-1}{\overline{r_{3}}}+\left[\overline{D z_{v}}-G_{2}^{2}{\overline{D z_{u}}}^{-1}\right]^{-1}\left(I+{\overline{D z_{u}}}^{-1} G_{2}\right)\left[{\overline{r_{4}}}^{-}{\overline{D z_{u}}}^{-1} \times\right.\right. \\
& \left.\left.\times\left(G_{2} \overline{r_{3}}-G_{2} U^{-1} \overline{r_{5}}\right)+V^{-1} \overline{r_{6}}\right]\right\} \\
& \overline{d v}=\left[\overline{D z_{v}}-G_{2}^{2} \overline{D z}_{u}^{-1}\right]^{-1}\left[\overline{r_{4}}+\overline{d y}+\overline{D z}_{u}^{-1}\left(-G_{2} \overline{r_{3}}+G_{2} \overline{d y}-G_{2} U^{-1} \overline{r_{5}}\right)+V^{-1} \overline{r_{6}}\right] \\
& \overline{d u}=\overline{D z}_{u}^{-1}\left(\overline{r_{3}}-\overline{d y}-G_{2} \overline{d v}+U^{-1} \overline{r_{5}}\right) \\
& \bar{d}_{u}=U^{-1}\left(\bar{r}_{5}-Z_{u} \overline{d u}\right) \\
& \overline{d z}_{v}=V^{-1}\left(\bar{r}_{6}-Z_{v} \overline{d v}\right) \\
& \hat{r_{1}}=-A^{t} y \\
& \hat{r_{2}}=-A x-u+v+b \\
& \hat{r_{3}}=\left(-G+Z_{u}-Y\right) e \\
& \hat{r_{4}}=\left(-G+Z_{v}+Y\right) e \\
& \hat{r_{5}}=-U Z_{u} e+\mu e-\bar{d} \bar{U} d \bar{Z}_{u} e \\
& \hat{r_{6}}=-V Z_{v} e+\mu e-d \bar{V} d \bar{Z}_{v} e \\
& \hat{r}=-\left\{\hat{r_{1}}-A^{t} \bar{D}\left[\hat{r}_{2}-\overline{D z}_{u}^{-1} \hat{r_{3}}+\left[\overline{D z_{v}}-G_{2}^{2} \overline{D z}_{u}{ }^{-1}\right]^{-1}\left(I+\overline{D z}_{u}{ }^{-1} G_{2}\right) \times\right.\right. \\
& \left.\left.\times\left[\hat{r}_{4}-\overline{D z}_{u}^{-1}\left(-G_{2} \hat{r}_{3}-G_{2} U^{-1} \hat{r_{5}}\right)+V^{-1} \hat{r_{6}}\right]\right]\right\} \\
& \hat{d x}=\left(A^{t} \bar{D} A\right)^{-1} \hat{r} \\
& \hat{d y}=\bar{D}\left\{{\hat{r_{2}}}_{-A \hat{d x}}-{\overline{D z_{u}}}^{-1} \hat{r_{3}}+\left[\overline{D z_{v}}-G_{2}^{2}{\overline{D z_{u}}}^{-1}\right]^{-1}\left(I+{\overline{D z_{u}}}^{-1} G_{2}\right)\left[{\hat{r_{4}}}^{-}{\overline{D z_{u}}}^{-1} \times\right.\right. \\
& \left.\left.\times\left(G_{2} \hat{r_{3}}-G_{2} U^{-1} \hat{r_{5}}\right)+V^{-1} \hat{r_{6}}\right]\right\} \\
& \hat{d v}=\left[\overline{D z}_{v}-G_{2}^{2} \overline{D z}_{u}^{-1}\right]^{-1}\left[\hat{r_{4}}+\hat{d y}+\overline{D z}_{u}^{-1}\left(-G_{2} \hat{r_{3}}+G_{2} \hat{d y}-G_{2} U^{-1} \hat{r_{5}}\right)+V^{-1} \hat{r_{6}}\right] \\
& \hat{d u}=\overline{D z}_{u}^{-1}\left(\hat{r_{3}}-\hat{d y}-G_{2} \hat{d v}+U^{-1} \hat{r_{5}}\right)
\end{aligned}
$$




$$
\begin{aligned}
\hat{d z_{u}} & =U^{-1}\left(\hat{r}_{5}-Z_{u} \hat{d u}\right) \\
\hat{d z_{v}} & =V^{-1}\left(\hat{r}_{6}-Z_{v} \hat{d v}\right) \\
\alpha & =\min \left\{\tau\left(\min _{d u_{i}<0}-\frac{u_{i}}{d u_{i}}\right), \tau\left(\min _{d v_{i}<0}-\frac{v_{i}}{d v_{i}}\right), \tau\left(\min _{d z_{u}<0}-\frac{z_{u i}}{d z_{u i}}\right), \tau\left(\min _{d z_{v i}<0}-\frac{z_{v i}}{d z_{v i}}\right), 1\right\} \\
u^{k+1} & =u^{k}+\alpha \hat{d u} \\
v^{k+1} & =v^{k}+\alpha \hat{d v} \\
x^{k+1} & =x^{k}+\alpha \hat{d x} \\
y^{k+1} & =y^{k}+\alpha \hat{d y} \\
z_{u}^{k+1} & =z_{u}^{k}+\alpha \hat{d z_{u}} \\
z_{v}^{k+1} & =z_{v}^{k}+\alpha \hat{d z_{v}} \\
\gamma & =u^{t} z_{u}+v^{t} z_{v} \\
\phi & =\frac{1}{m \sqrt{m}} \\
\mu & =\gamma \phi
\end{aligned}
$$

\subsubsection{Algumas Considerações}

O critério de convergência e o ponto inicial são os mesmos utilizados no método de pontos interiores primal-dual.

\subsection{Regressão Polinomial}

Consideremos o caso especial onde os dados são aproximados por um polinômio de grau $d=m-1$. Nesta situação, a matriz de restrição tem a mesma estrutura de

$$
V^{t}=\left(\begin{array}{cccc}
1 & 1 & \ldots & 1 \\
\beta_{1} & \beta_{2} & \ldots & \beta_{n} \\
\vdots & \vdots & \ddots & \vdots \\
\beta_{1}^{m-1} & \beta_{2}^{m-1} & \ldots & \beta_{n}^{m-1}
\end{array}\right)
$$

na qual é denominada matriz de Vandermonde retangular, onde $\left(\beta_{1}, \beta_{2}, \ldots, \beta_{n}\right)=b$.

Um procedimento para calcular o produto de $V$ por um vetor $x, w=V x$ é dado 
por

$$
\begin{aligned}
& w_{i} \leftarrow x_{m} \\
& w_{i} \leftarrow x_{j}+\beta_{i} w_{i}(\operatorname{para} j=m-1, \ldots, 1) .
\end{aligned}
$$

Além disso, o produto $H=V D^{*} V^{t}$, onde $D^{*}$ é uma matriz diagonal cujos elementos diagonais dependem do método, tem a estrutura especial de uma matriz de Hankel, seus elementos são constantes ao longo de suas antidiagonais. Isto é,

$$
H=\left(\begin{array}{cccc}
\eta_{1} & \eta_{2} & \ldots & \eta_{m} \\
\eta_{2} & \eta_{3} & \ldots & \vdots \\
\vdots & \vdots & \ddots & \eta_{2 m-2} \\
\eta_{m} & \ldots & \eta_{2 m-2} & \eta_{2 m-1}
\end{array}\right)
$$

Utilizando cuidadosamente esta estrutura, a complexidade computacional de uma iteração pode ser reduzida. A razão é que há algoritmos $O\left(m^{2}\right)$ para resolver sistemas de Hankel (por exemplo, [28]) e $H$ pode ser obtida em $O(n m)$ flops desde que somente $2 m-1$ elementos sejam calculados. Assim, a complexidade do pior caso de uma iteração é reduzida a $O\left(n^{2}\right)$ para o método GNCS devido à busca linear e $O(n m)$ para demais métodos. Observe que estas mudanças não afetam o procedimento de busca linear do GNCS. Por isso, quando $n$ torna-se muito maior que $m$ o desempenho do método GNCS pode piorar mais rápido do que os métodos de pontos interiores.

Observação 4.5.1 Flops é uma sigla para o número de operações de pontos flutuantes. No Matlab a função flops fornece um valor quase exato do número de operações de pontos flutuantes. Adições, subtrações, multiplicações e divisões são 1 flop cada se o resultado é real. Funções elementares também são 1 flop se o número é real.

Exemplo 4.5.1 Se $A$ e $B$ são matrizes reais $n \times n$, então

$$
\begin{gathered}
A+B=n^{2} \text { flops, } \\
A * B=2 * n^{3} \text { flops } .
\end{gathered}
$$


Embora o tempo das diferentes operações de ponto flutuante seja diferente, a proporção dessas operações é aproximadamente a mesma em todos os métodos vistos. Portanto, esta é uma medida convicta de comparação entre estes métodos.

Talvez o resultado mais surpreendente seja o fato de que não precisamos guardar nenhuma matriz em todos os métodos estudados, devido a estrutura especial de $V$. Os produtos matriz-vetor $V x$ ou $V^{t} y$ podem ser calculados na quantidade usual de flops sem que sejam armazenados. Do mesmo modo calculamos $H$ de $V$. Uma vez que $H$ tem somente $2 m-1$ elementos com valores diferentes, ele pode ser armazenado como um vetor. Além disso, é possível calcular sua inversa em $O\left(m^{2}\right)$ flops [28] e resolver simultaneamente o sistema linear. Assim, a matriz não é armazenada em nenhum procedimento. Note que o método de Trench [28] pode ser aplicado porque $H$ é definida positiva - assim todas as submatrizes principais são não-singulares. Um método onde pode ser conveniente (mas não necessário) armazenar uma matriz é o preditor-corretor. A metade inferior de $H^{-1}$ poderia ser armazenada para resolver o segundo sistema linear mais rapidamente.

Um procedimento para calcular o produto matriz-vetor $w=V x \operatorname{com} 2 n(m-1)$ flops é dado em (4.30) - note que $w_{i}=\sum_{j=1}^{m} \beta_{i}{ }^{j} x_{j}$. Um procedimento para obter $z=V^{t} y$ com $2 n m-n$ flops é dado a seguir - note que $z$ pode ser escrito como

$$
z=\sum_{i=1}^{n} y^{t}\left(1, \beta_{i}, \beta_{i}^{2}, \ldots, \beta_{i}^{m-1}\right)
$$

Procedimento para calcular $z=V^{t} y$

$$
\begin{aligned}
& z \leftarrow 0 \\
& \text { Para } i=1, \ldots, n \text { faça } \\
& \sigma \leftarrow y_{i} \\
& z_{1} \leftarrow z_{1}+y_{i} \\
& \text { Para } j=2, \ldots, m \text { faça } \\
& \sigma \leftarrow \beta_{i} \sigma \\
& z_{j} \leftarrow z_{j}+\sigma .
\end{aligned}
$$


Observe que, em geral, o produto matriz-vetor custa $2 \mathrm{~nm}$ flops. Este custo pode ser reduzido a $2 n m-n$ flops considerando que todos os componentes a primeira coluna de $V$ são 1. Neste contexto, o custo de calcular os valores $\left(\beta_{i}\right)^{j}$ também precisa ser considerado. Ele equivale a $n(m-2)$ flops. No entanto, este cálculo é feito somente uma vez, antes do início das iterações. Por isso, os procedimentos dados anteriormente não somente evitam o armazenamento da matriz $V$ mas de fato são ligeiramente mais baratos do que o procedimento geral.

Um procedimento semelhante para calcular a matriz $H$ é dado a seguir, onde $\delta_{i}^{*}$ é o i-ésimo elemento da diagonal de $D^{*}$. O procedimento custa $4 n m-3 n$ flops.

\section{Procedimento para calcular $H$}

$$
\begin{aligned}
& H \leftarrow 0 \\
& \text { Para } i=1, \ldots, n \text { faça } \\
& \sigma \leftarrow \delta_{i}^{*} \\
& \eta_{1} \leftarrow \eta_{1}+\delta_{i}^{*} \\
& \text { Para } j=2, \ldots, 2 m-1 \text { faça } \\
& \sigma \leftarrow \beta_{i} \sigma \\
& \eta_{j} \leftarrow \eta_{j}+\sigma .
\end{aligned}
$$

Há ainda outra melhoria para o problema de regressão polinomial. É muito fácil encontrar uma base sem armazenar qualquer matriz como anteriormente. Para calculá-la, é suficiente selecionar os $m$ menores resíduos $\left|r_{i}\right|$ de $r$ e interpolar os pontos correspondentes. Como os valores de $\beta_{i}$ são todos distintos, qualquer conjunto de colunas de $V$ levará a uma matriz não singular para interpolação.

Na implementação descrita em [21], os menores resíduos de $m$ são encontrados uti- 
lizando o algoritmo "heap" [26]. Uma vez são necessários somente os $m$ menores elementos, este algoritmo é adequado para nossa aplicação porque ele não precisa ordenar o vetor inteiro $r$. A complexidade computacional é $O(n \log (n))$. Após encontrado os pontos, a interpolação é calculada em $O\left(m^{2}\right)$ flops por diferenças divididas. 


\section{Capítulo 5}

\section{Resultados Computacionais}

Apresentamos neste capítulo os resultados computacionais obtidos pelos métodos de pontos interiores aplicados ao problema de regressão pela norma $L_{p}$ e a comparação com os resultados do método de convergência quadrática GNCS apresentado no Capítulo 3.

O método de pontos interiores barreira logarítmica, referido como BL, o método primal-dual barreira logarítmica, referido como PD, assim como o método preditor-corretor, referido como PC, o método primal-dual preditor-corretor, referido como PDPC, e o método de convergência quadrática GNCS foram implementados em Matlab 5.3, com o sistema operacional Windows Mileniun, processador Intel Celeron 700MHz.

Seguindo [13], aproximamos $f(z)$, calculado em $z=0, \frac{1}{m}, \ldots, 1$, por um polinômio de grau $n-1: p(z)=\sum_{j=1}^{n} x_{j} z^{j-1}$ tal que as normas $L_{p}$ são minimizadas. As duas funções testes utilizadas são

$$
f_{1}(z)=\sqrt{1+z}, \quad f_{2}(z)=e^{z}+ \begin{cases}5, & \text { se } 0.1<z<0.2 \\ 0 & \text { caso contrário. }\end{cases}
$$

A primeira função $f_{1}(z)$ é contínua, enquanto a segunda função $f_{2}(z)$ não é. Os elementos da matriz $A$ são compostos da seguinte forma: a primeira coluna é formada por um vetor unitário e a segunda coluna por elementos de 0 a 1 . O vetor $b$ é o valor da função obtido em cada ponto da segunda coluna de $A$. 
Os gráficos das funções são representados por:

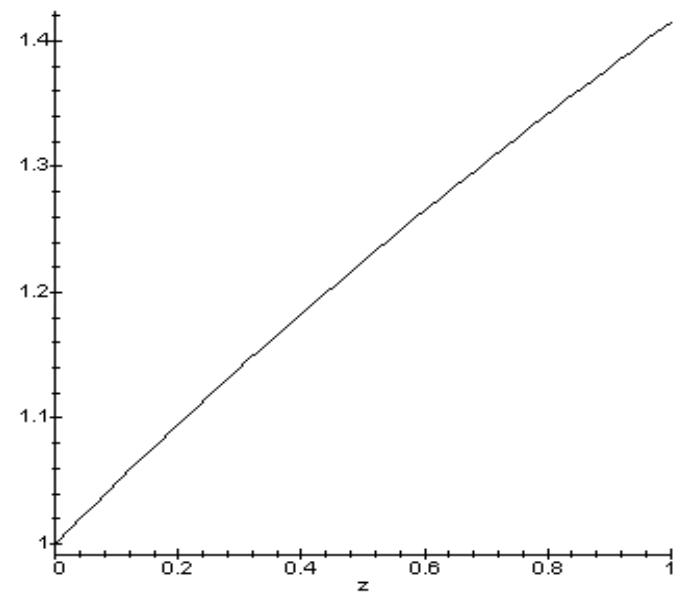

Figura 5.1: Função $f_{1}(z)$

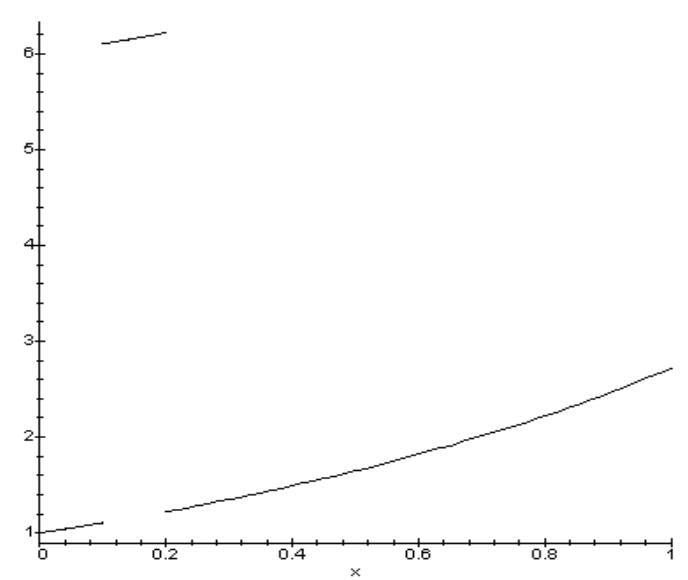

Figura 5.2: Função $f_{2}(z)$

Primeiramente, utilizando a função $f_{1}(z) \operatorname{com} n=2$, obtemos 


$$
A=\left[\begin{array}{cc}
1,0000 & 0,1000 \\
1,0000 & 0,2000 \\
1,0000 & 0,3000 \\
1,0000 & 0,4000 \\
1,0000 & 0,5000 \\
1,0000 & 0,6000 \\
1,0000 & 0,7000 \\
1,0000 & 0,8000 \\
1,0000 & 0,9000 \\
1,0000 & 1,0000
\end{array}\right] \text { e } b=\left[\begin{array}{l}
1,0488 \\
1,0954 \\
1,1402 \\
1,1832 \\
1,2247 \\
1,2649 \\
1,3038 \\
1,3416 \\
1,3784 \\
1,4142
\end{array}\right] .
$$

Agora, utilizando a função $f_{2}(z) \operatorname{com} n=2$, obtemos

$$
A=\left[\begin{array}{cc}
1,0000 & 0,1000 \\
1,0000 & 0,2000 \\
1,0000 & 0,3000 \\
1,0000 & 0,4000 \\
1,0000 & 0,5000 \\
1,0000 & 0,6000 \\
1,0000 & 0,7000 \\
1,0000 & 0,8000 \\
1,0000 & 0,9000 \\
1,0000 & 1,0000
\end{array}\right] \text { e } b=\left[\begin{array}{l}
1,1052 \\
1,2214 \\
1,3499 \\
1,4918 \\
1,6487 \\
1,8221 \\
2,0138 \\
2,2255 \\
2,4596 \\
2,7183
\end{array}\right] .
$$

As Tabelas 5.1 e 5.2 mostram os resultados obtidos pelos métodos de pontos interiores barreira logarítmica, preditor-corretor e o método GNCS utilizando $f_{1}(z)$ e $f_{2}(z)$ respectivamente. Utilizamos o parâmetro barreira $\mu=0,001$ e os critérios de parada $\epsilon=10^{-5}$ e $\epsilon_{1}=10^{-3}$. 


\begin{tabular}{|l|r|r|r|r|r|r|r|r|r|}
\hline \multirow{2}{*}{ P } & \multicolumn{4}{|c|}{ BL } & \multicolumn{3}{c|}{ PC } & \multicolumn{3}{c|}{ GNCS } \\
\cline { 2 - 10 } & It & Flops & FO & It & Flops & FO & It & Flops & FO \\
\hline 1 & 6 & 8132 & 0,0398 & 9 & 15650 & 0,0398 & 11 & 13759 & 0,0398 \\
\hline 1,1 & 1 & 1948 & 0,0245 & 1 & 2351 & 0,0245 & 11 & 13831 & 0,0238 \\
\hline 1,2 & 1 & 1952 & 0,0146 & 1 & 2359 & 0,0146 & 15 & 18831 & 0,0142 \\
\hline 1,3 & 1 & 1948 & 0,0091 & 1 & 2351 & 0,0090 & 6 & 7511 & 0,0085 \\
\hline 1,4 & 1 & 1948 & 0,0052 & 1 & 2351 & 0,0052 & 6 & 7511 & 0,0051 \\
\hline 1,5 & 1 & 1948 & 0,0031 & 1 & 2351 & 0,0031 & 6 & 7511 & 0,0031 \\
\hline 1,6 & 1 & 1952 & 0,0018 & 1 & 2359 & 0,0018 & 6 & 7491 & 0,0018 \\
\hline 1,7 & 1 & 1948 & 0,0011 & 1 & 2351 & 0,0011 & 5 & 6261 & 0,0011 \\
\hline 1,8 & 1 & 1948 & $6,5573 \mathrm{e}-4$ & 1 & 2351 & $6,5572 \mathrm{e}-4$ & 4 & 5031 & $6,5427 \mathrm{e}-4$ \\
\hline 1,9 & 1 & 1948 & $3,9242 \mathrm{e}-4$ & 1 & 2351 & $3,9242 \mathrm{e}-4$ & 4 & 5031 & $3,9208 \mathrm{e}-4$ \\
\hline
\end{tabular}

Tabela 5.1: Resultados computacionais utilizando a função $f_{1}(z)$.

\begin{tabular}{|l|r|r|c|r|r|r|r|r|c|}
\hline \multirow{2}{*}{ P } & \multicolumn{4}{|c|}{ BL } & \multicolumn{3}{c|}{ PC } & \multicolumn{3}{c|}{ GNCS } \\
\cline { 2 - 10 } & It & Flops & FO & It & Flops & FO & It & Flops & FO \\
\hline 1 & 6 & 8137 & 0,5284 & 2 & 4031 & 0,5558 & 7 & 8705 & 0,5280 \\
\hline 1,1 & 2 & 3205 & 0,4240 & 2 & 4031 & 0,4238 & 10 & 12431 & 0,4081 \\
\hline 1,2 & 2 & 3205 & 0,3242 & 2 & 4031 & 0,3239 & 8 & 9971 & 0,3162 \\
\hline 1,3 & 2 & 3205 & 0,2485 & 2 & 4031 & 0,2477 & 13 & 16331 & 0,2457 \\
\hline 1,4 & 2 & 3205 & 0,1923 & 2 & 4031 & 0,1903 & 10 & 12511 & 0,1902 \\
\hline 1,5 & 3 & 4476 & 0,1475 & 5 & 9105 & 0,1485 & 11 & 13741 & 0,1473 \\
\hline 1,6 & 3 & 4476 & 0,1146 & 4 & 7405 & 0,1474 & 9 & 11331 & 0,1141 \\
\hline 1,7 & 3 & 4476 & 0,0904 & 2 & 4011 & 0,1145 & 8 & 10081 & 0,0884 \\
\hline 1,8 & 2 & 3195 & 0,0694 & 2 & 4011 & 0,0693 & 6 & 7581 & 0,0685 \\
\hline 1,9 & 2 & 3195 & 0,0534 & 2 & 4011 & 0,0534 & 4 & 5011 & 0,0532 \\
\hline
\end{tabular}

Tabela 5.2: Resultados computacionais utilizando a função $f_{2}(z)$. 


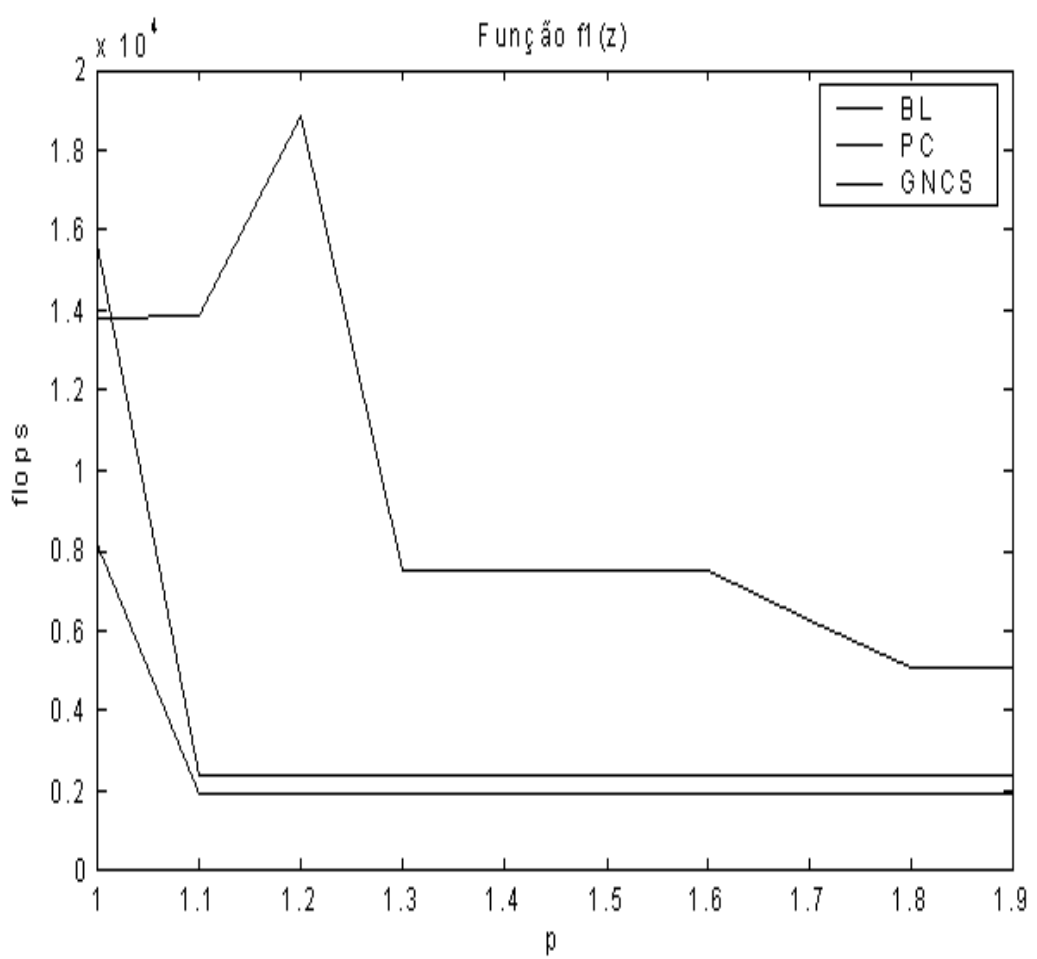

Figura 5.3: Referente à tabela 5.1

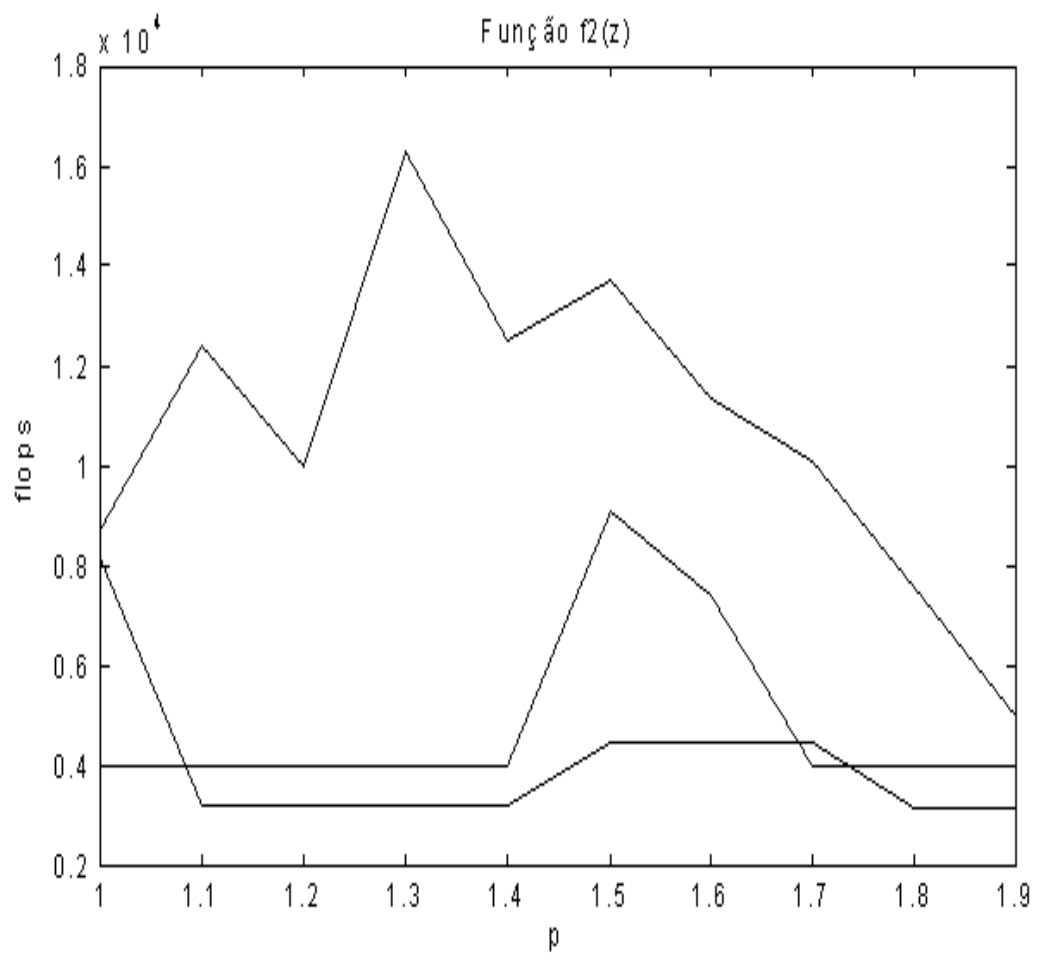

Figura 5.4: Referente à tabela 5.2 


\begin{tabular}{|l|r|r|r|r|r|r|}
\hline \multirow{2}{*}{ P } & \multicolumn{4}{|c|}{ PD } & \multicolumn{3}{c|}{ PDPC } \\
\cline { 2 - 7 } & It & Flops & FO & It & Flops & \multicolumn{1}{c|}{ FO } \\
\hline 1 & 1 & 2258 & 0,0385 & 1 & 2731 & 0,0401 \\
\hline 1,1 & 11 & 17868 & 0,0237 & 1 & 2881 & 0,0220 \\
\hline 1,2 & 9 & 14764 & 0,0141 & 1 & 2881 & 0,0143 \\
\hline 1,3 & 9 & 14764 & 0,0085 & 1 & 2881 & 0,0088 \\
\hline 1,4 & 8 & 13211 & 0,0051 & 6 & 13256 & 0,0041 \\
\hline 1,5 & 7 & 11659 & 0,0030 & 6 & 13256 & 0,0027 \\
\hline 1,6 & 7 & 11659 & 0,0018 & 1 & 2881 & 0,0020 \\
\hline 1,7 & 6 & 10108 & 0,0011 & 1 & 2881 & 0,0013 \\
\hline 1,8 & 5 & 8556 & $6,2227 \mathrm{e}-004$ & 5 & 11181 & $6,2483 \mathrm{e}-004$ \\
\hline 1,9 & 4 & 7004 & $3,7436 \mathrm{e}-004$ & 4 & 11181 & $3,7646 \mathrm{e}-004$ \\
\hline
\end{tabular}

Tabela 5.3: Utilizando a função $f_{1}(z): z_{u}=u, z_{v}=v$.

\begin{tabular}{|l|r|r|c|r|r|c|}
\hline \multirow{2}{*}{ P } & \multicolumn{3}{|c|}{ PD } & \multicolumn{3}{c|}{ PDPC } \\
\cline { 2 - 7 } & It & Flops & FO & It & Flops & FO \\
\hline 1 & 1 & 2262 & 0,5589 & 1 & 2735 & 0,5406 \\
\hline 1,1 & 6 & 10112 & 0,3834 & 7 & 15335 & 0,3033 \\
\hline 1,2 & 7 & 11664 & 0,3112 & 4 & 9110 & 0,2944 \\
\hline 1,3 & 5 & 8560 & 0,2261 & 4 & 9110 & 0,1152 \\
\hline 1,4 & 5 & 8560 & 0,1830 & 4 & 9110 & 0,1302 \\
\hline 1,5 & 5 & 8560 & 0,1446 & 4 & 9110 & 0,1089 \\
\hline 1,6 & 5 & 8560 & 0,1138 & 4 & 9110 & 0,0918 \\
\hline 1,7 & 7 & 11664 & 0,0879 & 4 & 9110 & 0,0769 \\
\hline 1,8 & 7 & 11664 & 0,0682 & 4 & 9110 & 0,0630 \\
\hline 1,9 & 7 & 11664 & 0,0530 & 4 & 9110 & 0,0507 \\
\hline
\end{tabular}

Tabela 5.4: Utilizando a função $f_{2}(z): z_{u}=u, z_{v}=v$. 


\begin{tabular}{|l|r|r|r|r|r|r|}
\hline \multirow{2}{*}{ P } & \multicolumn{4}{|c|}{ PD } & \multicolumn{3}{c|}{ PDPC } \\
\cline { 2 - 7 } & It & Flops & \multicolumn{1}{c|}{ FO } & It & Flops & \multicolumn{1}{c|}{ FO } \\
\hline 1 & 3 & 5342 & 0,0363 & 4 & 8906 & 36,7509 \\
\hline 1,1 & 3 & 5452 & 0,0219 & 4 & 9076 & 0,0265 \\
\hline 1,2 & 3 & 5452 & 0,0134 & 4 & 9076 & 0,0138 \\
\hline 1,3 & 7 & 11660 & 0,0082 & 4 & 9076 & 0,0080 \\
\hline 1,4 & 5 & 8556 & 0,0043 & 4 & 9076 & 0,0044 \\
\hline 1,5 & 5 & 8556 & 0,0028 & 4 & 9076 & 0,0027 \\
\hline 1,6 & 6 & 10108 & 0,0017 & 6 & 13226 & 0,0018 \\
\hline 1,7 & 7 & 11660 & $9,9510 \mathrm{e}-004$ & 7 & 15301 & 0,0015 \\
\hline 1,8 & 7 & 11660 & $5,8523 \mathrm{e}-004$ & 7 & 15301 & $6,0710 \mathrm{e}-004$ \\
\hline 1,9 & 7 & 11660 & $3,8314 \mathrm{e}-004$ & 7 & 15301 & $3,2959 \mathrm{e}-004$ \\
\hline
\end{tabular}

Tabela 5.5: Utilizando a função $f_{1}(z)$ e $z_{u}=z_{v}=e$.

\begin{tabular}{|r|r|r|c|r|r|r|}
\hline \multirow{2}{*}{$\mathrm{P}$} & \multicolumn{3}{|c|}{$\mathrm{PD}$} & \multicolumn{3}{c|}{ PDPC } \\
\cline { 2 - 7 } & It & Flops & FO & It & Flops & FO \\
\hline 1 & 3 & 5346 & 0,4520 & 5 & 10985 & $1,1636 \mathrm{e}+003$ \\
\hline 1,1 & 5 & 8560 & 0,3443 & 5 & 11155 & 0,2934 \\
\hline 1,2 & 5 & 8560 & 0,2832 & 4 & 9080 & 0,2760 \\
\hline 1,3 & 6 & 10112 & 0,2324 & 6 & 13230 & 0,2122 \\
\hline 1,4 & 5 & 8560 & 0,1754 & 6 & 13230 & 0,1744 \\
\hline 1,5 & 6 & 10112 & 0,1396 & 6 & 13230 & 0,1371 \\
\hline 1,6 & 6 & 10112 & 0,1072 & 6 & 13230 & 0,1070 \\
\hline 1,7 & 6 & 10112 & 0,0838 & 6 & 13230 & 0,0835 \\
\hline 1,8 & 6 & 10112 & 0,0665 & 6 & 13230 & 0,0652 \\
\hline 1,9 & 7 & 11664 & 0,0507 & 6 & 13230 & 0,0510 \\
\hline
\end{tabular}

Tabela 5.6: Utilizando a função $f_{2}(z)$ e $z_{u}=z_{v}=e$. 


\begin{tabular}{|r|r|r|r|r|r|r|}
\hline \multirow{2}{*}{$\mathrm{P}$} & \multicolumn{4}{|c|}{ PD } & \multicolumn{3}{c|}{ PDPC } \\
\cline { 2 - 7 } & It & Flops & FO & It & Flops & \multicolumn{1}{c|}{ FO } \\
\hline 1 & 3 & 5274 & 0,0347 & 4 & 8838 & 32,5980 \\
\hline 1,1 & 6 & 10040 & 0,0230 & 5 & 11083 & 0,0230 \\
\hline 1,2 & 8 & 13144 & 0,0136 & 5 & 11083 & 0,0122 \\
\hline 1,3 & 7 & 11592 & 0,0082 & 5 & 11083 & 0,0076 \\
\hline 1,4 & 5 & 8488 & 0,0043 & 5 & 11083 & 0,0047 \\
\hline 1,5 & 5 & 8488 & 0,0030 & 5 & 11083 & 0,0029 \\
\hline 1,6 & 5 & 8488 & 0,0019 & 5 & 11083 & 0,0019 \\
\hline 1,7 & 4 & 6936 & 0,0010 & 5 & 11083 & 0,0013 \\
\hline 1,8 & 6 & 10040 & $6,5256 \mathrm{e}-004$ & 7 & 15233 & $5,8673 \mathrm{e}-004$ \\
\hline 1,9 & 6 & 10040 & $3,8549 \mathrm{e}-004$ & 7 & 15233 & $3,2485 \mathrm{e}-004$ \\
\hline
\end{tabular}

Tabela 5.7: Utilizando a função $f_{1}(z)$ e $z_{u}=((\lambda+1) / 2)$ e e $z_{v}=((\lambda-1) / 2) e$.

\begin{tabular}{|l|r|r|c|r|r|r|}
\hline \multirow{2}{*}{ P } & \multicolumn{4}{|c|}{ PD } & \multicolumn{3}{|c|}{ PDPC } \\
\cline { 2 - 7 } & It & Flops & FO & It & Flops & FO \\
\hline 1 & 9 & 14590 & 0,5714 & 3 & 6767 & 415,5724 \\
\hline 1,1 & 6 & 10004 & 0,3796 & 6 & 13162 & 0,7215 \\
\hline 1,2 & 4 & 6940 & 0,2900 & 7 & 15237 & 0,2789 \\
\hline 1,3 & 4 & 6940 & 0,2270 & 5 & 11087 & 0,1912 \\
\hline 1,4 & 4 & 6940 & 0,1776 & 7 & 15237 & 0,1702 \\
\hline 1,5 & 4 & 6940 & 0,1406 & 7 & 15237 & 0,1394 \\
\hline 1,6 & 4 & 6940 & 0,1115 & 7 & 15237 & 0,1087 \\
\hline 1,7 & 4 & 6940 & 0,0873 & 7 & 15237 & 0,0844 \\
\hline 1,8 & 4 & 6940 & 0,0647 & 7 & 15237 & 0,0656 \\
\hline 1,9 & 4 & 6940 & 0,0501 & 7 & 15237 & 0,0511 \\
\hline
\end{tabular}

Tabela 5.8: Utilizando a função $f_{2}(z)$ e $z_{u}=((\lambda+1) / 2)$ e e $z_{v}=((\lambda-1) / 2) e$. 
Podemos verificar nas tabelas 5.1 e 5.2 que, tanto em relação as iterações quanto em relação ao número de flops, os métodos de pontos interiores apresentaram melhores resultados que o método GNCS.

Nas tabelas de 5.3 a 5.8 encontram-se os resultados obtidos pelo método primaldual, cujos valores iniciais de $z_{u}$ e $z_{v}$ foram variados conforme indicado nas tabelas.

Os métodos de pontos interiores para as funções $f_{1}(z)$ e $f_{2}(z)$ apresentam desempenho computacional superior que o método GNCS, tanto em relação às iterações quanto ao número de flops.

Na maioria dos casos, $z_{u}=z_{v}=e$ apresentam melhores resultados, utilizando tanto a função $f_{1}(z)$ quanto a função $f_{2}(z)$. Desta forma, utilizamos esse valor inicial para $z_{u}$ e $z_{v}$ como padrão para todos os testes computacionais realizados.

Realizamos agora testes computacionais com várias funções nas quais encontramse descritas a seguir. Foram utilizados números aleatórios entre [0,1], obtidos através de um gerador, totalizando 1000 pontos em cada caso.

Utilizamos primeiramente a função $\sin x$. Nas tabelas 5.9 e 5.10 aproximamos a função por uma reta, com $\epsilon=10^{-5}$ e $\epsilon_{1}=10^{-3}$. Nas tabelas 5.11 e 5.12 aproximamos a função por um polinômio de grau 4 , com $\epsilon=10^{-10}$ e $\epsilon_{1}=10^{-8}$.

Utilizamos agora a função $\sinh x$. Nas tabelas 5.13 e 5.14 aproximamos a função por uma parábola, com $\epsilon=10^{-6}$ e $\epsilon_{1}=10^{-3}$. Nas tabelas 5.15 e 5.16 aproximamos a função por um polinômio de grau 5 , com $\epsilon=10^{-10}$ e $\epsilon_{1}=10^{-8}$.

Nas tabelas 5.17 e 5.18 utilizamos a aproximação da função $\ln x$ por um polinômio de grau 3, com $\epsilon=10^{-8}$ e $\epsilon_{1}=10^{-6}$. Nas tabelas 5.19 e 5.20 utilizamos a aproximação da função por um polinômio de grau $6, \operatorname{com} \epsilon=10^{-10}$ e $\epsilon_{1}=10^{-8}$. 
Nas tabelas 5.21 e 5.22 utilizamos a aproximação da função exp $x$ por um polinômio de grau 4, com $\epsilon=10^{-10}$ e $\epsilon_{1}=10^{-8}$. Nas tabelas 5.23 e 5.24 utilizamos a aproximação da função por um polinômio de grau $8, \operatorname{com} \epsilon=10^{-10}$ e $\epsilon_{1}=10^{-8}$.

Nas tabelas 5.25 e 5.26 utilizamos a aproximação da função exp $x^{2}$ por um polinômio de grau 5, com $\epsilon=10^{-10}$ e $\epsilon_{1}=10^{-8}$. Nas tabelas 5.27 e 5.28 utilizamos a aproximação da função por um polinômio de grau 10, com $\epsilon=10^{-10}$ e $\epsilon_{1}=10^{-8}$.

Utilizamos agora um problema real de grande porte no qual estamos principalmente interessados em comparar o tempo de CPU em segundos e número de iterações necessários para a convergência dos métodos. O conjunto contém os dados "Prime Rate" (PR). O conjunto de dados compreende valores dos juros diários ao longo de 40 anos, finais-desemanas e feriados não foram considerados, totalizando 10958 valores observados. Para evitar problemas de estabilidade numérica dos métodos, os dados (observados) foram normalizados no intervalo [0,1]. Estes experimentos foram realizados em uma estação Sun Blade 100 com Matlab 6.0, assim, não foi possível estimar o número de flops. Utilizamos o parâmetro barreira $\mu=0,001$ e os critérios de parada $\epsilon=10^{-10}, \epsilon_{1}=10^{-8}$. Os resultados obtidos se encontram nas tabelas a seguir.

Podemos verificar que os métodos de pontos interiores apresentam desempenho computacional superior que o método GNCS, tanto em relação ao tempo computacional quanto à validade da solução.

Os métodos primal-dual barreira logarítmica e primal-dual preditor-corretor convergem para problemas de grande porte em menos iterações e com esforço por iteração muito menor que o método GNCS utilizando o critério de convergência (3.24). Podemos também verificar que os métodos de pontos interiores primal-dual barreira logarítmica e primal-dual preditor corretor apresentam desempenho computacional superior aos métodos de pontos interiores barreira logarítmica e preditor-corretor. O método GNCS utilizando 
o critério de convergência (3.23) proposto em [13] não converge mesmo após 100 iterações. 


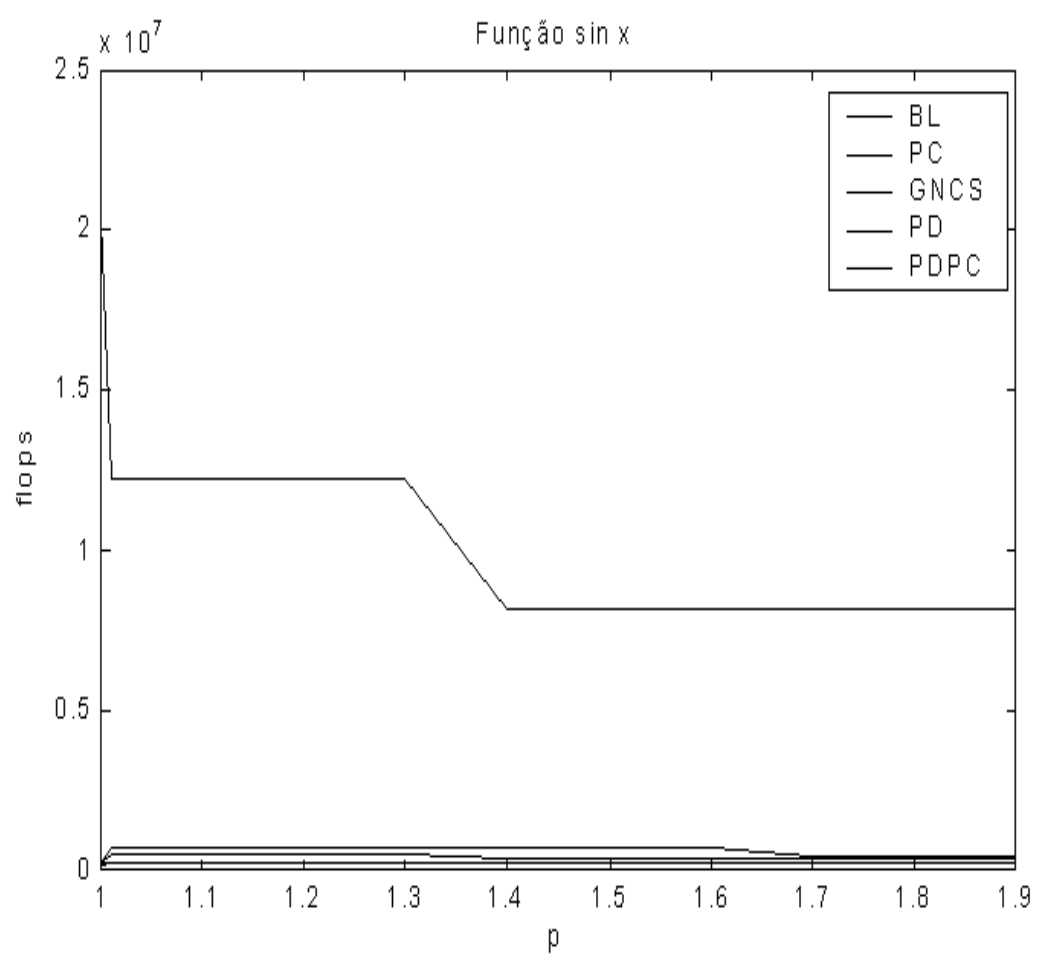

Figura 5.5: Referente às tabelas 5.9 e 5.10

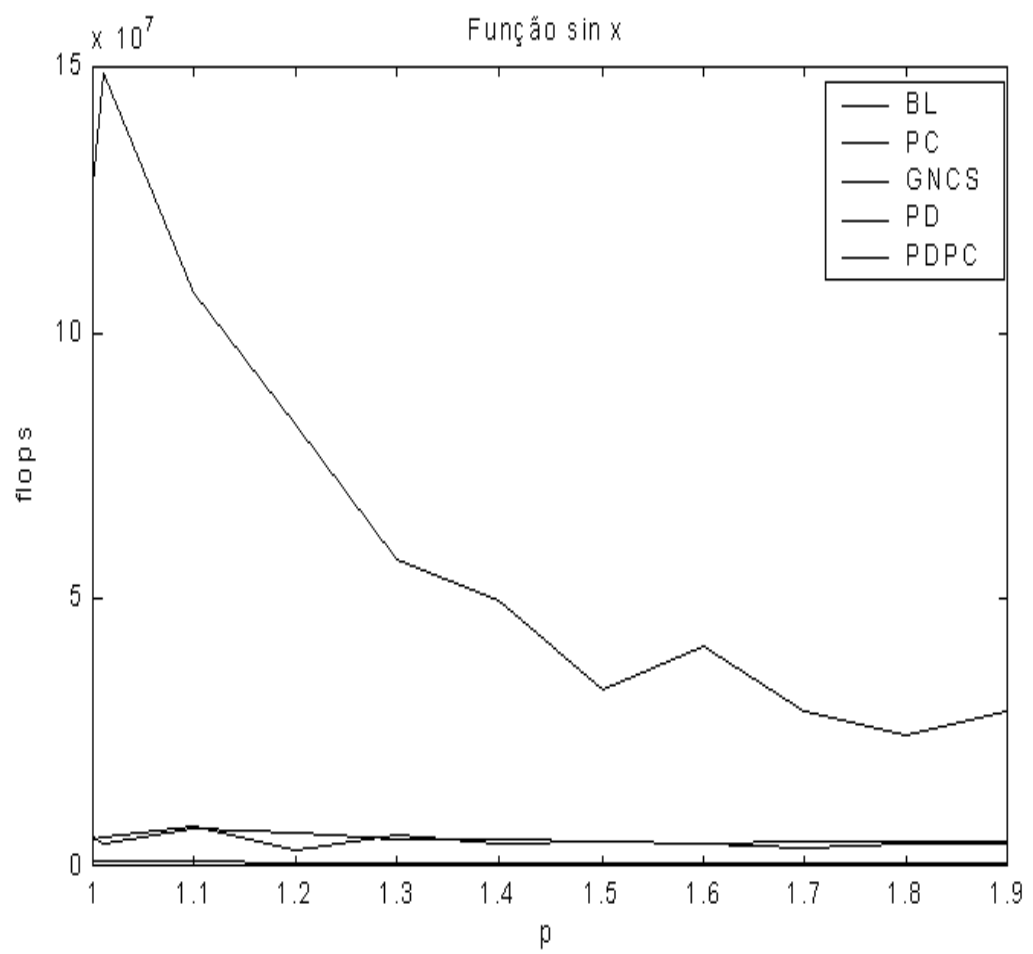

Figura 5.6: Referente às tabelas 5.11 e 5.12 


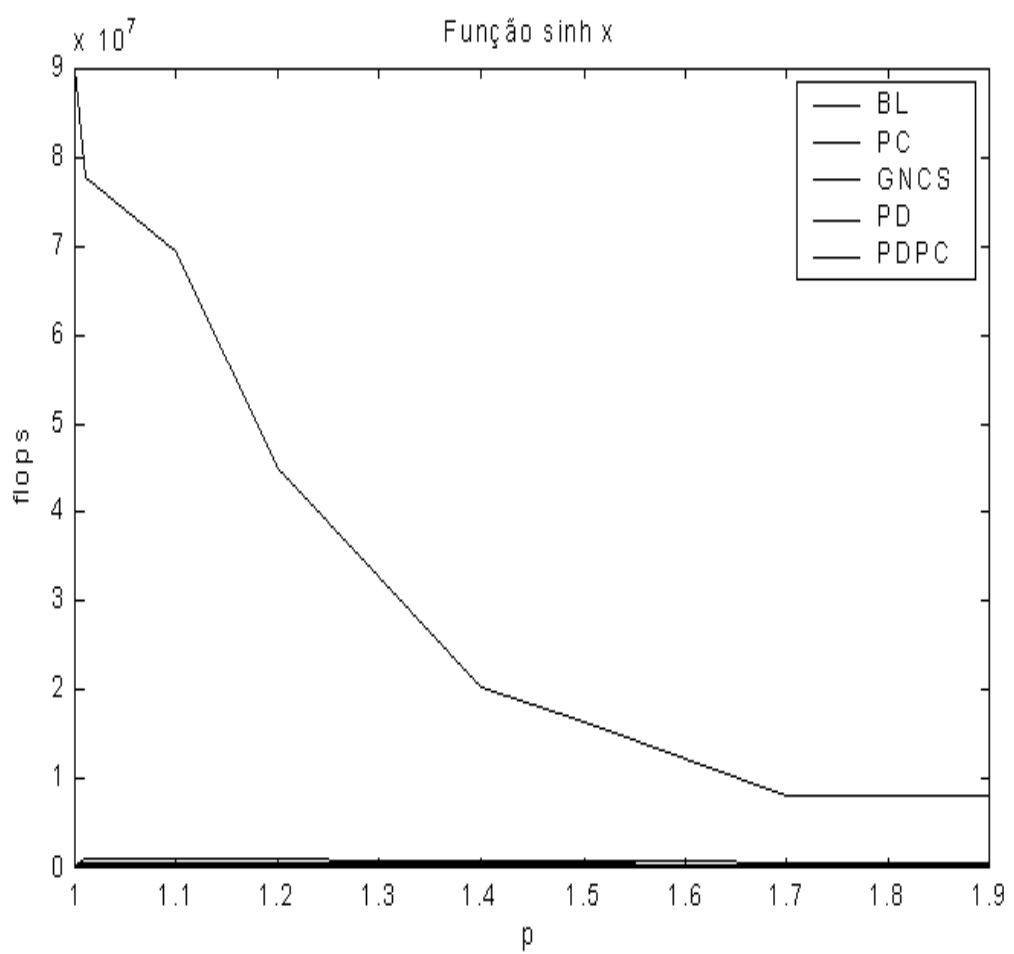

Figura 5.7: Referente às tabelas 5.13 e 5.14

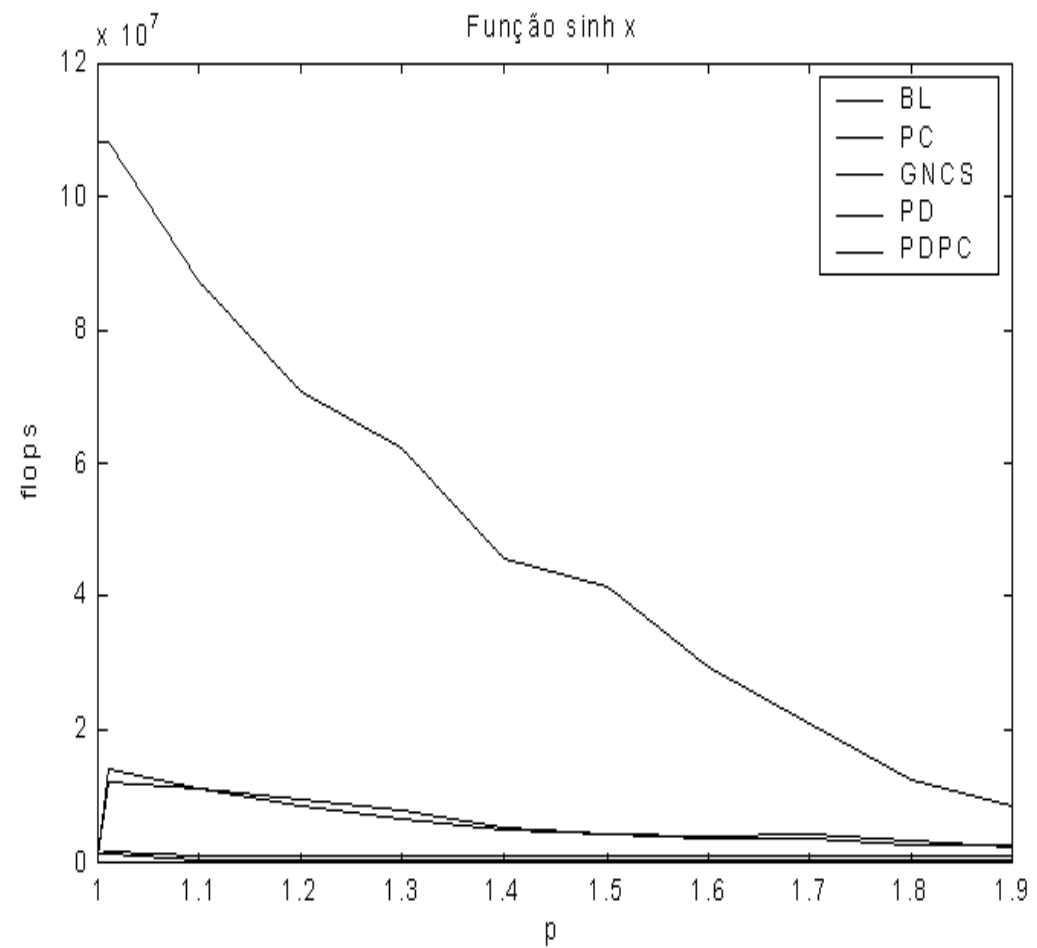

Figura 5.8: Referente às tabelas 5.15 e 5.16 


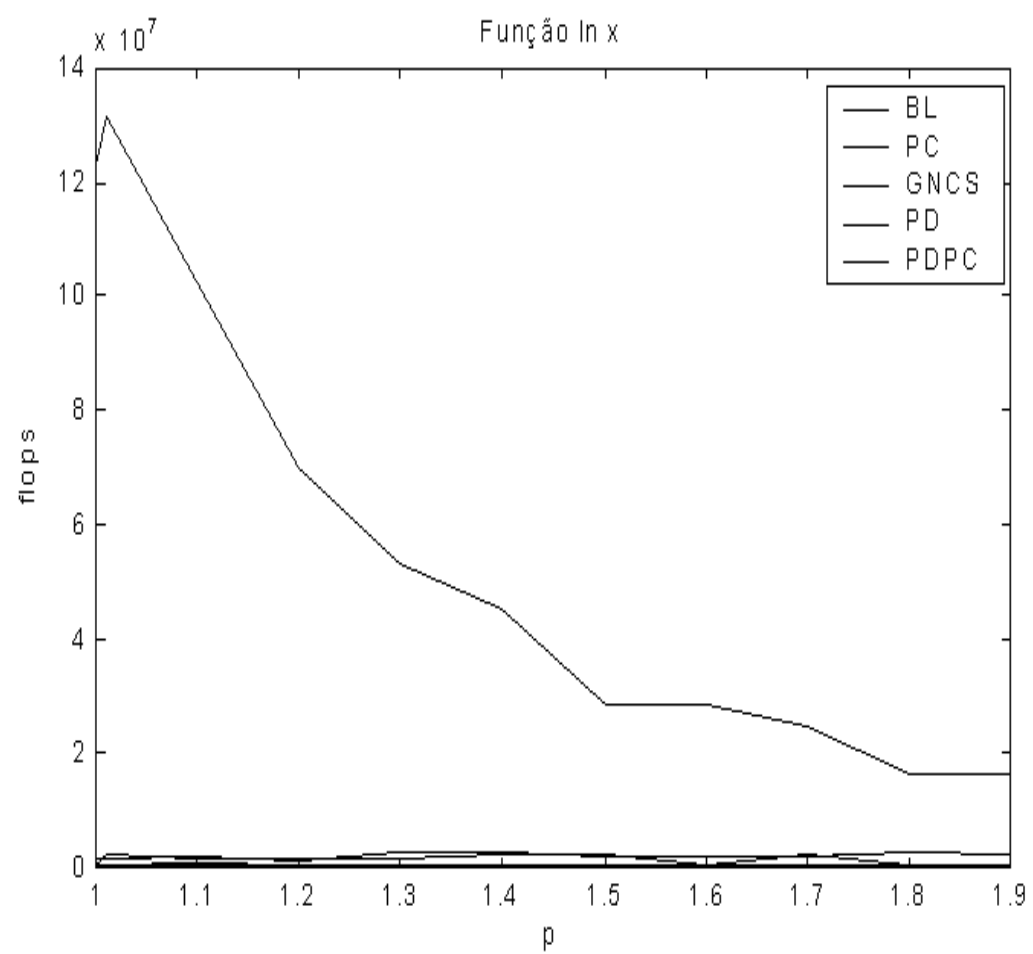

Figura 5.9: Referente às tabelas 5.17 e 5.18

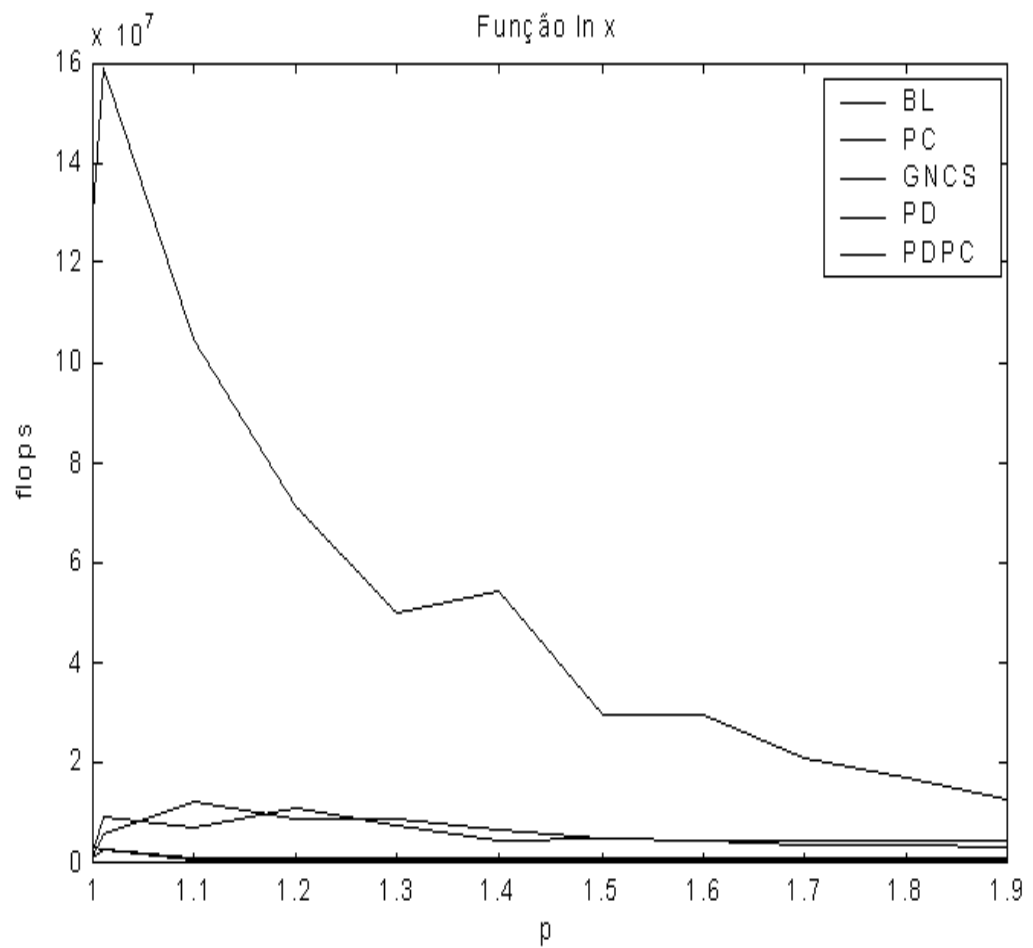

Figura 5.10: Referente às tabelas 5.19 e 5.20 


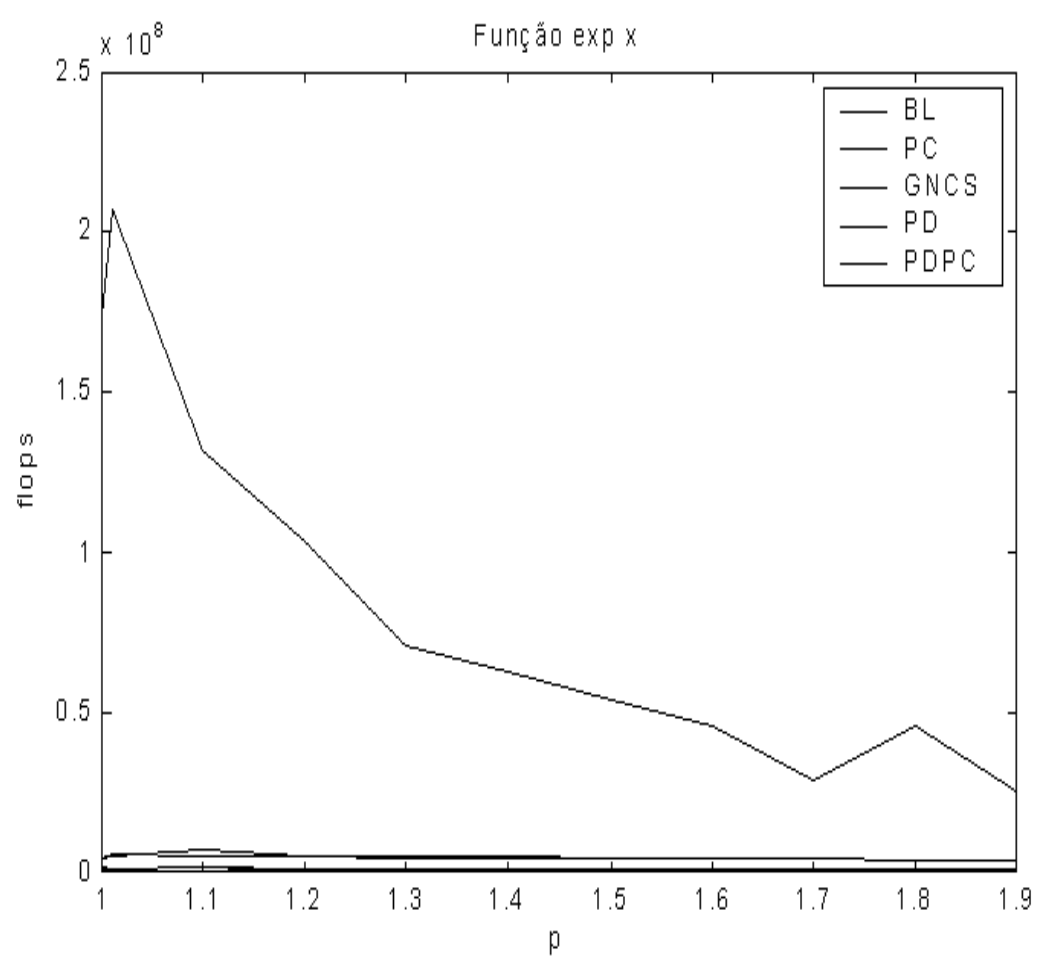

Figura 5.11: Referente às tabelas 5.21 e 5.22

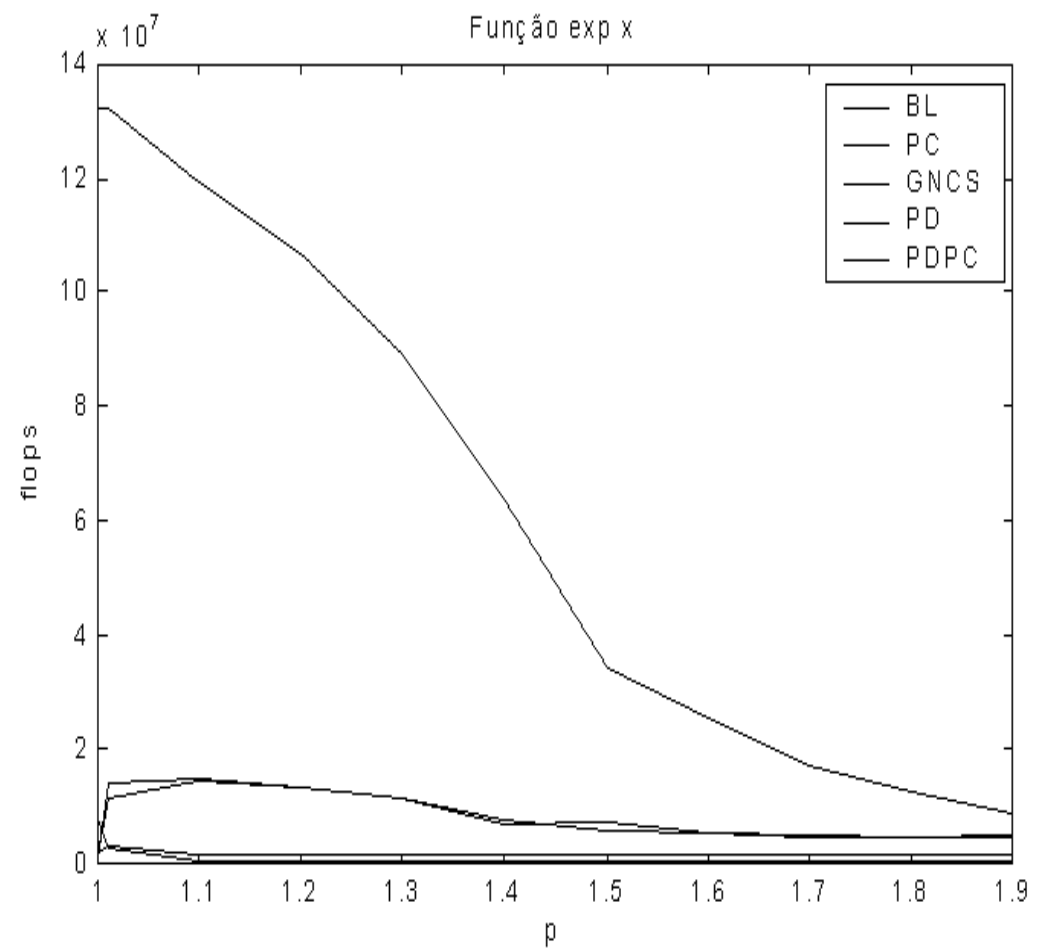

Figura 5.12: Referente às tabelas 5.23 e 5.24 


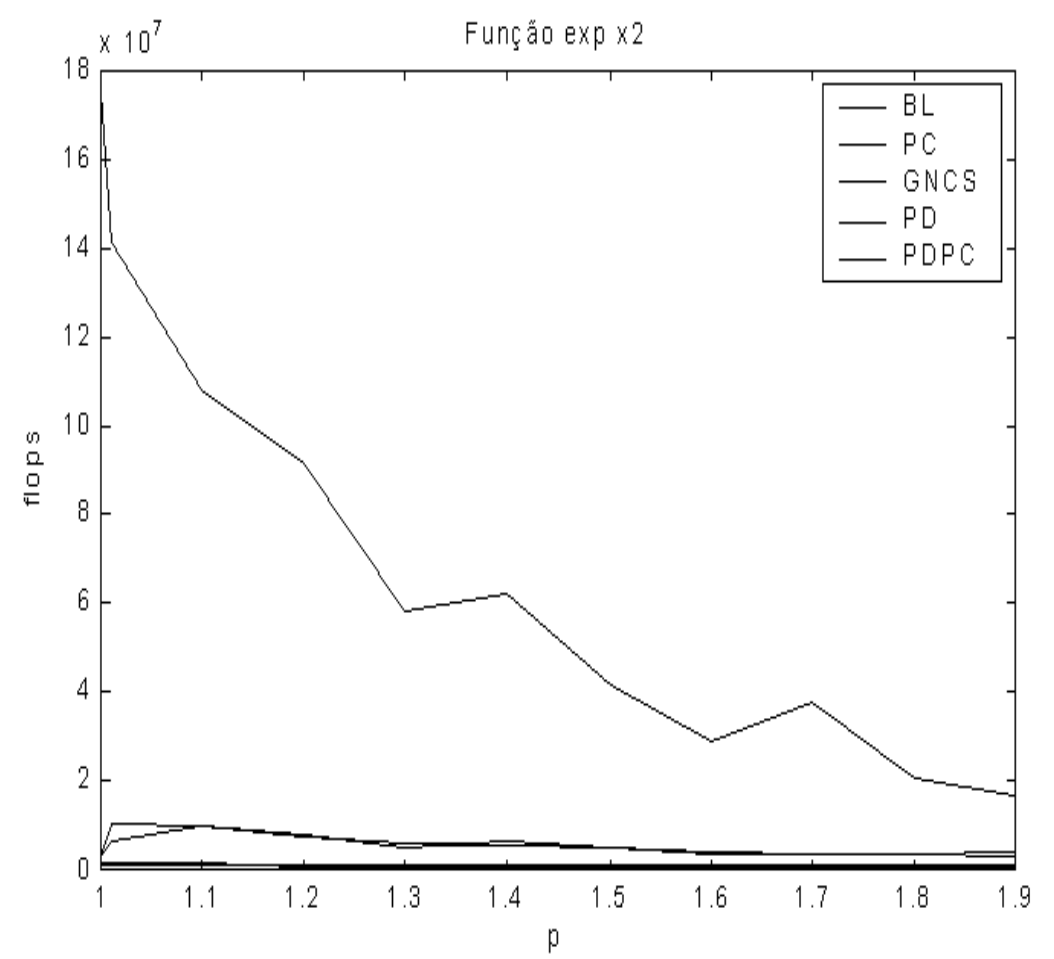

Figura 5.13: Referente às tabelas 5.25 e 5.26

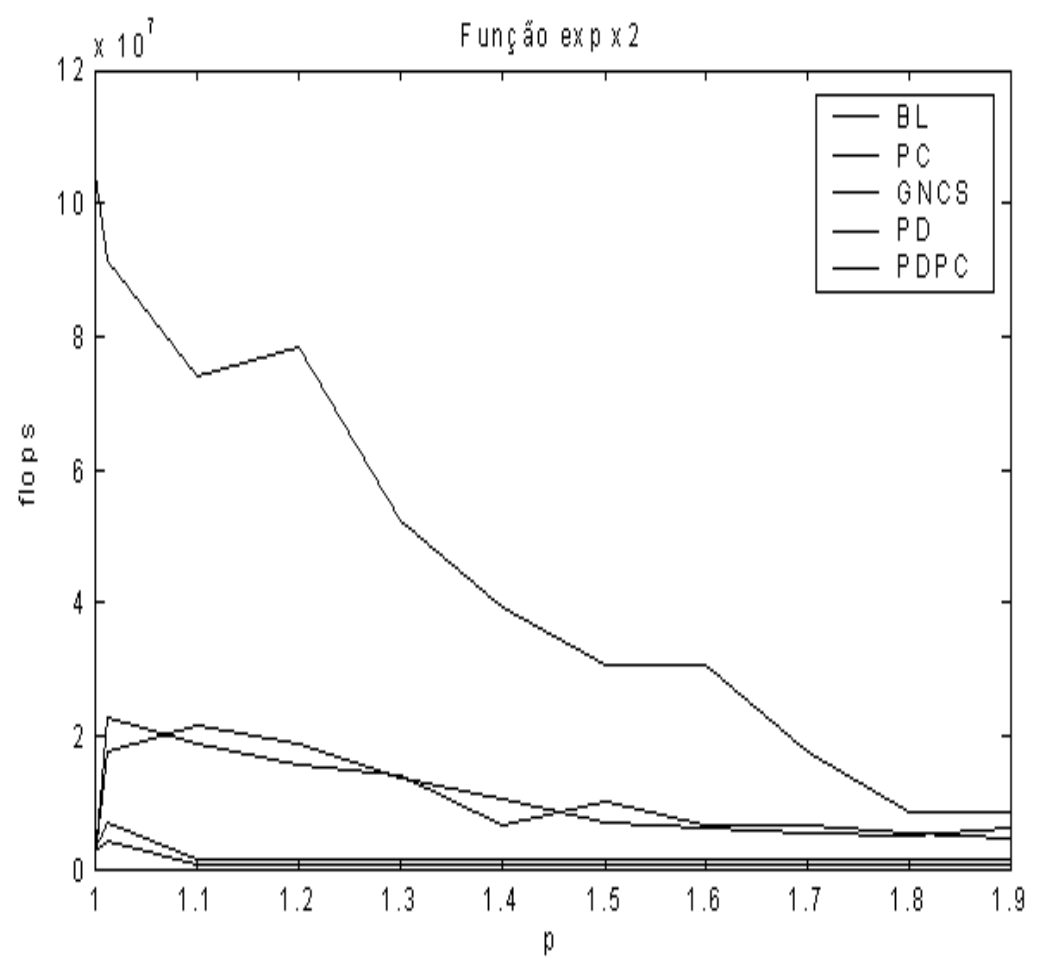

Figura 5.14: Referente às tabelas 5.27 e 5.28 


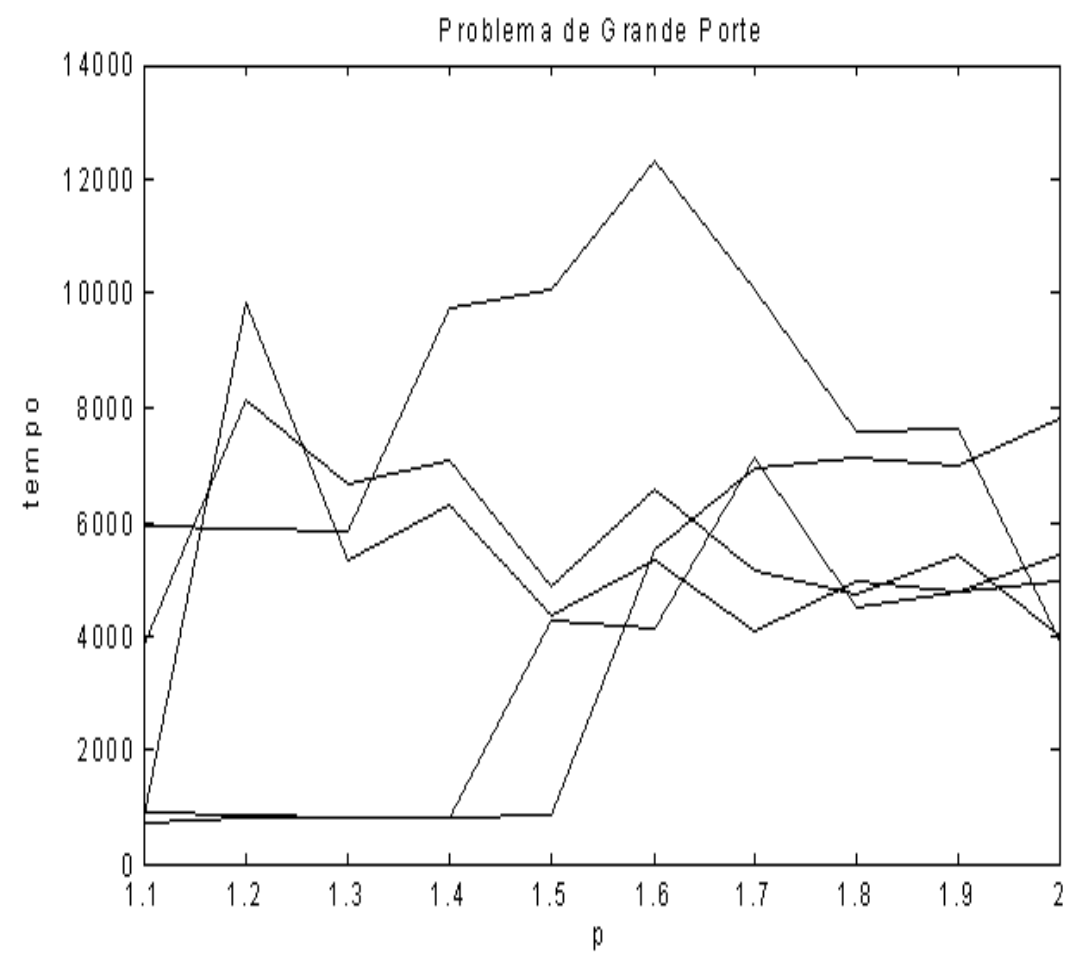

Figura 5.15: Referente às tabelas 5.29, 5.30 e 5.31 


\begin{tabular}{|l|c|c|c|c|c|c|c|c|c|}
\hline \multirow{2}{*}{ P } & \multicolumn{4}{|c|}{ BL } & \multicolumn{3}{c|}{ PC } & \multicolumn{3}{c|}{ GNCS } \\
\cline { 2 - 10 } & It & Flops & FO & It & Flops & FO & It & Flops & FO \\
\hline 1 & 1 & 173431 & 53,8331 & 1 & 206431 & 53,8358 & 5 & 20358892 & 52,6350 \\
\hline 1,01 & 1 & 184420 & 52,3824 & 1 & 223414 & 52,3853 & 3 & 12218940 & 51,5613 \\
\hline 1,1 & 1 & 184420 & 41,0034 & 1 & 223414 & 41,0143 & 3 & 12220938 & 40,4386 \\
\hline 1,2 & 1 & 184420 & 31,2088 & 1 & 223414 & 31,2164 & 3 & 12221937 & 30,8568 \\
\hline 1,3 & 1 & 184420 & 23,8680 & 1 & 223414 & 23,8713 & 3 & 12227935 & 23,6701 \\
\hline 1,4 & 1 & 184420 & 18,3006 & 1 & 223414 & 18,3007 & 2 & 8152962 & 18,2464 \\
\hline 1,5 & 1 & 184420 & 14,0700 & 1 & 223414 & 14,0689 & 2 & 8152962 & 14,0596 \\
\hline 1,6 & 1 & 184420 & 10,8544 & 1 & 223414 & 10,8546 & 2 & 8152962 & 10,8577 \\
\hline 1,7 & 1 & 184420 & 8,4143 & 1 & 223414 & 8,4106 & 2 & 8152962 & 8,3998 \\
\hline 1,8 & 1 & 184420 & 6,5097 & 1 & 223414 & 6,5091 & 2 & 8152962 & 6,5079 \\
\hline 1,9 & 1 & 184410 & 5,0798 & 1 & 223394 & 5,0821 & 2 & 8152962 & 5,0485 \\
\hline
\end{tabular}

Tabela 5.9: Resultados computacionais utilizando a função sin $x$.

\begin{tabular}{|l|c|c|c|c|c|c|}
\hline \multirow{2}{*}{ P } & \multicolumn{3}{|c|}{ PD } & \multicolumn{3}{c|}{ PDPC } \\
\cline { 2 - 7 } & It & Flops & FO & It & Flops & FO \\
\hline 1 & 1 & 211403 & 53,6410 & 1 & 252395 & 53,8168 \\
\hline 1,01 & 3 & 518240 & 50,9050 & 3 & 663194 & 51,4332 \\
\hline 1,1 & 3 & 518240 & 39,8398 & 3 & 663194 & 40,1028 \\
\hline 1,2 & 3 & 518240 & 30,4629 & 3 & 663194 & 30,6103 \\
\hline 1,3 & 3 & 518240 & 23,3502 & 3 & 663194 & 23,4631 \\
\hline 1,4 & 2 & 370316 & 18,2127 & 3 & 663194 & 18,0339 \\
\hline 1,5 & 2 & 370316 & 14,0075 & 3 & 663194 & 13,8896 \\
\hline 1,6 & 2 & 370316 & 10,7954 & 3 & 663194 & 10,7203 \\
\hline 1,7 & 2 & 370316 & 8,3373 & 2 & 466286 & 8,3971 \\
\hline 1,8 & 2 & 370316 & 6,4546 & 2 & 466286 & 6,4974 \\
\hline 1,9 & 2 & 370316 & 5,0164 & 2 & 466286 & 5,0365 \\
\hline
\end{tabular}

Tabela 5.10: Resultados computacionais utilizando a função $\sin x$. 


\begin{tabular}{|l|r|r|r|r|r|r|r|r|r|}
\hline \multirow{2}{*}{ P } & \multicolumn{4}{|c|}{ BL } & \multicolumn{3}{c|}{ PC } & \multicolumn{3}{c|}{ GNCS } \\
\cline { 2 - 10 } & It & Flops & FO & It & Flops & FO & It & Flops & FO \\
\hline 1 & 4 & 830435 & 0,0563 & 4 & 1029057 & 0,0563 & 31 & 128045643 & 0,0535 \\
\hline 1,01 & 4 & 841349 & 0,0511 & 4 & 1046040 & 0,0511 & 36 & 148697069 & 0,0487 \\
\hline 1,1 & 4 & 841424 & 0,0486 & 4 & 1046040 & 0,0506 & 26 & 107397216 & 0,0207 \\
\hline 1,2 & 3 & 650346 & 0,0083 & 1 & 319344 & 0,0083 & 20 & 82614708 & 0,0080 \\
\hline 1,3 & 3 & 650346 & 0,0032 & 1 & 319344 & 0,0032 & 14 & 57832200 & 0,0031 \\
\hline 1,4 & 3 & 650346 & 0,0012 & 1 & 319344 & 0,0012 & 12 & 49570032 & 0,0012 \\
\hline 1,5 & 3 & 650346 & $4,7876 \mathrm{e}-004$ & 1 & 319344 & $4,7876 \mathrm{e}-004$ & 8 & 33049692 & $4,7164 \mathrm{e}-004$ \\
\hline 1,6 & 3 & 650346 & $1,8576 \mathrm{e}-004$ & 1 & 319344 & $1,8576 \mathrm{e}-004$ & 10 & 41309862 & $1,8395 \mathrm{e}-004$ \\
\hline 1,7 & 3 & 650346 & $7,2239 \mathrm{e}-005$ & 1 & 319344 & $7,2239 \mathrm{e}-005$ & 7 & 28919607 & $7,1833 \mathrm{e}-005$ \\
\hline 1,8 & 3 & 650346 & $2,8154 \mathrm{e}-005$ & 1 & 319344 & $2,8154 \mathrm{e}-005$ & 6 & 24789522 & $2,8083 \mathrm{e}-005$ \\
\hline 1,9 & 3 & 650346 & $1,0997 \mathrm{e}-005$ & 1 & 319344 & $1,0997 \mathrm{e}-005$ & 7 & 28919607 & $1,0993 \mathrm{e}-005$ \\
\hline
\end{tabular}

Tabela 5.11: Resultados computacionais utilizando a função $\sin x$.

\begin{tabular}{|l|c|c|r|r|c|r|}
\hline \multirow{2}{*}{ P } & \multicolumn{4}{|c|}{ PD } & \multicolumn{3}{c|}{ PDPC } \\
\cline { 2 - 7 } & It & Flops & \multicolumn{1}{c|}{ FO } & It & Flops & \multicolumn{1}{c|}{ FO } \\
\hline 1 & 24 & 5356415 & 0,0404 & 17 & 4847493 & 0,4899 \\
\hline 1,01 & 17 & 3827026 & 0,0416 & 19 & 5426872 & 0,0465 \\
\hline 1,1 & 31 & 6907782 & 0,0207 & 27 & 7676456 & 0,0207 \\
\hline 1,2 & 28 & 6247620 & 0,0080 & 11 & 3177288 & 0,0079 \\
\hline 1,3 & 22 & 4927296 & 0,0031 & 20 & 5708069 & 0,0031 \\
\hline 1,4 & 21 & 4707242 & 0,0012 & 14 & 4020882 & 0,0012 \\
\hline 1,5 & 19 & 4267134 & $4,7161 \mathrm{e}-004$ & 15 & 4302080 & $4,7164 \mathrm{e}-004$ \\
\hline 1,6 & 17 & 3827026 & $1,8393 \mathrm{e}-004$ & 14 & 4020882 & $1,8395 \mathrm{e}-004$ \\
\hline 1,7 & 16 & 3606972 & $7,1820 \mathrm{e}-005$ & 15 & 4302080 & $7,1829 \mathrm{e}-005$ \\
\hline 1,8 & 18 & 4047080 & $2,8083 \mathrm{e}-005$ & 15 & 4302080 & $2,8081 \mathrm{e}-005$ \\
\hline 1,9 & 18 & 4047080 & $1,0990 \mathrm{e}-005$ & 15 & 4302080 & $1,0989 \mathrm{e}-005$ \\
\hline
\end{tabular}

Tabela 5.12: Resultados computacionais utilizando a função $\sin x$. 


\begin{tabular}{|c|c|c|c|c|c|c|c|c|c|}
\hline \multirow{2}{*}{ P } & \multicolumn{4}{|c|}{ BL } & \multicolumn{3}{c|}{ PC } & \multicolumn{3}{c|}{ GNCS } \\
\cline { 2 - 10 } & It & Flops & FO & It & Flops & FO & It & Flops & FO \\
\hline 1 & 1 & 197214 & 3,0649 & 1 & 234247 & 3,0653 & 22 & 89910914 & 2,9616 \\
\hline 1,01 & 1 & 208203 & 2,8977 & 1 & 251230 & 2,8980 & 19 & 77644934 & 2,8023 \\
\hline 1,1 & 1 & 208203 & 1,7496 & 1 & 251230 & 1,7500 & 17 & 69501913 & 1,7061 \\
\hline 1,2 & 1 & 208203 & 1,0003 & 1 & 251230 & 1,0018 & 11 & 44973949 & 0,9851 \\
\hline 1,3 & 1 & 208216 & 0,6713 & 1 & 251256 & 0,5828 & 8 & 32709967 & 0,5699 \\
\hline 1,4 & 1 & 208203 & 0,3355 & 1 & 251230 & 0,3360 & 5 & 20445985 & 0,3303 \\
\hline 1,5 & 1 & 208203 & 0,1939 & 1 & 251230 & 0,1939 & 4 & 16357991 & 0,1917 \\
\hline 1,6 & 1 & 208203 & 0,1123 & 1 & 251230 & 0,1123 & 3 & 12269997 & 0,1115 \\
\hline 1,7 & 1 & 208203 & 0,0652 & 1 & 251230 & 0,0652 & 2 & 8182003 & 0,0649 \\
\hline 1,8 & 1 & 208203 & 0,0379 & 1 & 251230 & 0,0379 & 2 & 8182003 & 0,0378 \\
\hline 1,9 & 1 & 208203 & 0,0221 & 1 & 251230 & 0,0221 & 2 & 8182003 & 0,0221 \\
\hline
\end{tabular}

Tabela 5.13: Resultados computacionais utilizando a função $\sinh x$.

\begin{tabular}{|l|c|c|c|c|c|c|}
\hline \multirow{2}{*}{ P } & \multicolumn{3}{|c|}{ PD } & \multicolumn{3}{c|}{ PDPC } \\
\cline { 2 - 7 } & It & Flops & FO & It & Flops & FO \\
\hline 1 & 1 & 235186 & 3,0653 & 1 & 280211 & 3,0814 \\
\hline 1,01 & 3 & 582077 & 2,7854 & 4 & 960098 & 2,7398 \\
\hline 1,1 & 3 & 582077 & 1,6715 & 4 & 960098 & 1,6181 \\
\hline 1,2 & 2 & 414126 & 0,9828 & 4 & 960098 & 0,9176 \\
\hline 1,3 & 2 & 414126 & 0,5639 & 3 & 739130 & 0,5679 \\
\hline 1,4 & 2 & 414126 & 0,3243 & 3 & 739130 & 0,3268 \\
\hline 1,5 & 2 & 414126 & 0,1871 & 3 & 739130 & 0,1885 \\
\hline 1,6 & 1 & 246175 & 0,1113 & 3 & 739130 & 0,1089 \\
\hline 1,7 & 1 & 246175 & 0,0646 & 2 & 518162 & 0,0648 \\
\hline 1,8 & 1 & 246175 & 0,0375 & 2 & 518162 & 0,0376 \\
\hline 1,9 & 1 & 246175 & 0,0218 & 1 & 297194 & 0,0221 \\
\hline
\end{tabular}

Tabela 5.14: Resultados computacionais utilizando a função $\sinh x$. 


\begin{tabular}{|c|r|r|r|r|r|r|r|r|c|}
\hline \multirow{2}{*}{ P } & \multicolumn{4}{|c|}{ BL } & \multicolumn{4}{c|}{ PC } & \multicolumn{3}{c|}{ GNCS } \\
\cline { 2 - 10 } & It & Flops & FO & It & Flops & FO & It & Flops & FO \\
\hline 1 & 6 & 1409089 & 0,0020 & 5 & 1456213 & 0,0034 & 26 & 108127218 & $2,8144 \mathrm{e}-004$ \\
\hline 1,01 & 8 & 1866402 & 0,0021 & 5 & 1473196 & 0,0029 & 26 & 108127218 & $2,4268 \mathrm{e}-004$ \\
\hline 1,1 & 4 & 973754 & $6,4133 \mathrm{e}-005$ & 1 & 359524 & $6,4125 \mathrm{e}-005$ & 21 & 87334410 & $6,4056 \mathrm{e}-005$ \\
\hline 1,2 & 4 & 973754 & $1,4638 \mathrm{e}-005$ & 1 & 359524 & $1,4638 \mathrm{e}-005$ & 17 & 70699764 & $1,4626 \mathrm{e}-005$ \\
\hline 1,3 & 4 & 973754 & $3,3517 \mathrm{e}-006$ & 1 & 359524 & $3,3517 \mathrm{e}-006$ & 15 & 62397426 & $3,3496 \mathrm{e}-006$ \\
\hline 1,4 & 4 & 973754 & $7,6957 \mathrm{e}-007$ & 1 & 359524 & $7,6957 \mathrm{e}-007$ & 11 & 45758784 & $7,6920 \mathrm{e}-007$ \\
\hline 1,5 & 4 & 973754 & $1,7716 \mathrm{e}-007$ & 1 & 359524 & $1,7716 \mathrm{e}-007$ & 10 & 41602620 & $1,7710 \mathrm{e}-007$ \\
\hline 1,6 & 4 & 973754 & $4,0882 \mathrm{e}-008$ & 1 & 359524 & $4,0882 \mathrm{e}-008$ & 7 & 29127135 & $4,0873 \mathrm{e}-008$ \\
\hline 1,7 & 4 & 973754 & $9,4557 \mathrm{e}-009$ & 1 & 359524 & $9,4557 \mathrm{e}-009$ & 5 & 20806815 & $9,4544 \mathrm{e}-009$ \\
\hline 1,8 & 4 & 973754 & $2,1917 \mathrm{e}-009$ & 1 & 359524 & $2,1917 \mathrm{e}-009$ & 3 & 12486495 & $2,1915 \mathrm{e}-009$ \\
\hline 1,9 & 4 & 973754 & $5,0902 \mathrm{e}-010$ & 1 & 359524 & $5,0902 \mathrm{e}-010$ & 2 & 8326335 & $5,0901 \mathrm{e}-010$ \\
\hline
\end{tabular}

Tabela 5.15: Resultados computacionais utilizando a função sinh $x$.

\begin{tabular}{|l|r|r|c|r|c|r|}
\hline \multirow{2}{*}{ P } & \multicolumn{4}{|c}{ PD } & \multicolumn{3}{c|}{ PDPC } \\
\cline { 2 - 7 } & It & Flops & FO & It & Flops & FO \\
\hline 1 & 4 & 1087665 & $7,0296 \mathrm{e}-004$ & 3 & 1023273 & 0,0021 \\
\hline 1,01 & 47 & 11940588 & $2,4268 \mathrm{e}-004$ & 44 & 14053000 & $2,4205 \mathrm{e}-004$ \\
\hline 1,1 & 44 & 11184174 & $6,4056 \mathrm{e}-005$ & 35 & 11196543 & $6,4056 \mathrm{e}-005$ \\
\hline 1,2 & 38 & 9671346 & $1,4626 \mathrm{e}-005$ & 26 & 8340088 & $1,4613 \mathrm{e}-005$ \\
\hline 1,3 & 30 & 7654242 & $3,3496 \mathrm{e}-006$ & 21 & 6753168 & $3,3496 \mathrm{e}-006$ \\
\hline 1,4 & 21 & 5385000 & $7,6908 \mathrm{e}-007$ & 15 & 4848863 & $7,6920 \mathrm{e}-007$ \\
\hline 1,5 & 17 & 4376448 & $1,7700 \mathrm{e}-007$ & 13 & 4214096 & $1,7709 \mathrm{e}-007$ \\
\hline 1,6 & 14 & 3620034 & $4,0617 \mathrm{e}-008$ & 12 & 3896712 & $4,0849 \mathrm{e}-008$ \\
\hline 1,7 & 13 & 3367896 & $9,1948 \mathrm{e}-009$ & 13 & 4214096 & $9,3905 \mathrm{e}-009$ \\
\hline 1,8 & 11 & 2863620 & $1,9742 \mathrm{e}-009$ & 10 & 3261944 & $2,0763 \mathrm{e}-009$ \\
\hline 1,9 & 11 & 2863620 & $8,0135 \mathrm{e}-010$ & 8 & 2627176 & $4,2201 \mathrm{e}-010$ \\
\hline
\end{tabular}

Tabela 5.16: Resultados computacionais utilizando a função $\sinh x$. 


\begin{tabular}{|c|c|c|r|r|c|r|r|r|r|}
\hline \multirow{2}{*}{ P } & \multicolumn{4}{|c|}{ BL } & \multicolumn{4}{c|}{ PC } & \multicolumn{3}{c|}{ GNCS } \\
\cline { 2 - 10 } & It & Flops & FO & It & Flops & FO & It & Flops & FO \\
\hline 1 & 1 & 225032 & 0,2150 & 1 & 266117 & 0,2150 & 30 & 123189942 & 0,2113 \\
\hline 1,01 & 1 & 236021 & 0,1974 & 1 & 283100 & 0,1974 & 32 & 131402004 & 0,1948 \\
\hline 1,1 & 1 & 236021 & 0,1024 & 3 & 703204 & 0,0974 & 25 & 102659787 & 0,0939 \\
\hline 1,2 & 2 & 399038 & 0,0436 & 1 & 283100 & 0,0436 & 17 & 69811539 & 0,0419 \\
\hline 1,3 & 2 & 399048 & 0,0193 & 1 & 283120 & 0,0193 & 13 & 53387415 & 0,0187 \\
\hline 1,4 & 2 & 399038 & 0,0086 & 1 & 283100 & 0,0086 & 11 & 45175353 & 0,0084 \\
\hline 1,5 & 2 & 399038 & 0,0038 & 1 & 283100 & 0,0038 & 7 & 28751229 & 0,0037 \\
\hline 1,6 & 2 & 399038 & 0,0017 & 1 & 283100 & 0,0017 & 7 & 28751229 & 0,0017 \\
\hline 1,7 & 2 & 399038 & $7,5975 \mathrm{e}-004$ & 1 & 283100 & $7,5975 \mathrm{e}-004$ & 6 & 24645198 & $7,5509 \mathrm{e}-004$ \\
\hline 1,8 & 2 & 399038 & $3,4055 \mathrm{e}-004$ & 1 & 283100 & $3,4055 \mathrm{e}-004$ & 4 & 16433136 & $3,3961 \mathrm{e}-004$ \\
\hline 1,9 & 2 & 399038 & $1,5301 \mathrm{e}-004$ & 1 & 283100 & $1,5301 \mathrm{e}-004$ & 4 & 16433136 & $1,5290 \mathrm{e}-004$ \\
\hline
\end{tabular}

Tabela 5.17: Resultados computacionais utilizando a função $\ln x$.

\begin{tabular}{|l|r|c|r|r|r|r|}
\hline \multirow{2}{*}{$\mathrm{P}$} & \multicolumn{4}{|c|}{ PD } & \multicolumn{3}{c|}{ PDPC } \\
\cline { 2 - 7 } & It & Flops & FO & It & Flops & \multicolumn{1}{c|}{ FO } \\
\hline 1 & 9 & 1798948 & 1,0909 & 1 & 312081 & 0,2234 \\
\hline 1,01 & 9 & 1809937 & 0,1478 & 10 & 2570622 & 0,1609 \\
\hline 1,1 & 10 & 2001930 & 0,0709 & 6 & 1574374 & 0,0675 \\
\hline 1,2 & 5 & 1041965 & 0,0315 & 6 & 1574374 & 0,0306 \\
\hline 1,3 & 14 & 2769902 & 0,0161 & 7 & 1823436 & 0,0132 \\
\hline 1,4 & 14 & 2769902 & 0,0074 & 10 & 2570622 & 0,0083 \\
\hline 1,5 & 11 & 2193923 & 0,0029 & 10 & 2570622 & 0,0037 \\
\hline 1,6 & 11 & 2193923 & 0,0013 & 1 & 329064 & 0,0017 \\
\hline 1,7 & 11 & 2193923 & $6,3001 \mathrm{e}-004$ & 9 & 2321560 & $6,6829 \mathrm{e}-004$ \\
\hline 1,8 & 14 & 2769902 & $2,9689 \mathrm{e}-004$ & 1 & 329064 & $3,3951 \mathrm{e}-004$ \\
\hline 1,9 & 12 & 2385916 & $1,4304 \mathrm{e}-004$ & 1 & 329064 & $1,5254 \mathrm{e}-004$ \\
\hline
\end{tabular}

Tabela 5.18: Resultados computacionais utilizando a função $\ln x$. 


\begin{tabular}{|c|r|c|r|r|r|r|r|r|c|}
\hline \multirow{2}{*}{ P } & \multicolumn{4}{|c|}{ BL } & \multicolumn{4}{c|}{ PC } & \multicolumn{3}{c|}{ GNCS } \\
\cline { 2 - 10 } & It & Flops & FO & It & Flops & FO & It & Flops & FO \\
\hline 1 & 12 & 3185273 & 0,0085 & 3 & 1023989 & 0,0240 & 31 & 129907258 & $6,3055 \mathrm{e}-004$ \\
\hline 1,01 & 10 & 2677716 & 0,0067 & 8 & 2634282 & 0,0143 & 38 & 159247112 & $5,4881 \mathrm{e}-004$ \\
\hline 1,1 & 4 & 1122078 & $1,6283 \mathrm{e}-004$ & 1 & 403648 & $1,6284 \mathrm{e}-004$ & 25 & 104765662 & $1,5737 \mathrm{e}-004$ \\
\hline 1,2 & 4 & 1122078 & $4,0864 \mathrm{e}-005$ & 1 & 403648 & $4,0864 \mathrm{e}-005$ & 17 & 71243534 & $3,9361 \mathrm{e}-005$ \\
\hline 1,3 & 4 & 1122078 & $1,0157 \mathrm{e}-005$ & 1 & 403648 & $1,0157 \mathrm{e}-005$ & 12 & 50296200 & $9,8624 \mathrm{e}-006$ \\
\hline 1,4 & 4 & 1122078 & $2,5314 \mathrm{e}-006$ & 1 & 403648 & $2,5314 \mathrm{e}-006$ & 13 & 54488464 & $2,4760 \mathrm{e}-006$ \\
\hline 1,5 & 4 & 1122078 & $6,3268 \mathrm{e}-007$ & 1 & 403648 & $6,3268 \mathrm{e}-007$ & 7 & 29342872 & $6,2279 \mathrm{e}-007$ \\
\hline 1,6 & 4 & 1122078 & $1,5855 \mathrm{e}-007$ & 1 & 403648 & $1,5855 \mathrm{e}-007$ & 7 & 29342872 & $1,5694 \mathrm{e}-007$ \\
\hline 1,7 & 4 & 1122078 & $3,9834 \mathrm{e}-008$ & 1 & 403648 & $3,9834 \mathrm{e}-008$ & 5 & 20962340 & $3,9605 \mathrm{e}-008$ \\
\hline 1,8 & 4 & 1122078 & $1,0033 \mathrm{e}-008$ & 1 & 403648 & $1,0033 \mathrm{e}-008$ & 4 & 16775071 & $1,0008 \mathrm{e}-008$ \\
\hline 1,9 & 4 & 1122078 & $2,5334 \mathrm{e}-009$ & 1 & 403648 & $2,5334 \mathrm{e}-009$ & 3 & 12581808 & $2,5318 \mathrm{e}-009$ \\
\hline
\end{tabular}

Tabela 5.19: Resultados computacionais utilizando a função $\ln x$.

\begin{tabular}{|l|r|r|r|r|r|r|}
\hline \multirow{2}{*}{ P } & \multicolumn{4}{|c|}{ PD } & \multicolumn{3}{c|}{ PDPC } \\
\cline { 2 - 7 } & It & Flops & FO & It & Flops & FO \\
\hline 1 & 4 & 1235989 & 0,0023 & 6 & 2220769 & 0,0278 \\
\hline 1,01 & 19 & 5570713 & $4,0722 \mathrm{e}-004$ & 26 & 9390312 & $5,4880 \mathrm{e}-004$ \\
\hline 1,1 & 42 & 12200440 & $1,5737 \mathrm{e}-004$ & 19 & 6886916 & $1,5415 \mathrm{e}-004$ \\
\hline 1,2 & 30 & 8741452 & $3,9361 \mathrm{e}-005$ & 30 & 10820824 & $3,9361 \mathrm{e}-005$ \\
\hline 1,3 & 30 & 8741452 & $9,8624 \mathrm{e}-006$ & 21 & 7602172 & $9,8624 \mathrm{e}-006$ \\
\hline 1,4 & 23 & 6723709 & $2,4759 \mathrm{e}-006$ & 12 & 4383520 & $2,4683 \mathrm{e}-006$ \\
\hline 1,5 & 16 & 4705966 & $6,2206 \mathrm{e}-007$ & 13 & 4741148 & $6,2276 \mathrm{e}-007$ \\
\hline 1,6 & 14 & 4129468 & $1,5526 \mathrm{e}-007$ & 12 & 4383520 & $1,5686 \mathrm{e}-007$ \\
\hline 1,7 & 13 & 3841219 & $3,7686 \mathrm{e}-008$ & 11 & 4025892 & $3,9457 \mathrm{e}-008$ \\
\hline 1,8 & 12 & 3552970 & $8,5652 \mathrm{e}-009$ & 11 & 4025892 & $9,9167 \mathrm{e}-009$ \\
\hline 1,9 & 11 & 3264721 & $2,8376 \mathrm{e}-009$ & 11 & 4025892 & $2,4049 \mathrm{e}-009$ \\
\hline
\end{tabular}

Tabela 5.20: Resultados computacionais utilizando a função $\ln x$. 


\begin{tabular}{|c|r|r|r|r|r|r|r|r|r|}
\hline \multirow{2}{*}{ P } & \multicolumn{4}{|c|}{ BL } & \multicolumn{3}{c|}{ PC } & \multicolumn{3}{c|}{ GNCS } \\
\cline { 2 - 10 } & It & Flops & FO & It & Flops & FO & It & Flops & FO \\
\hline 1 & 5 & 1021313 & 0,0141 & 5 & 1271239 & 0,0141 & 42 & 173476578 & 0,0135 \\
\hline 1,01 & 4 & 841374 & 0,0121 & 4 & 1045690 & 0,0121 & 50 & 206524257 & 0,0121 \\
\hline 1,1 & 7 & 1414608 & 0,1648 & 6 & 1530454 & 0,1174 & 32 & 132175728 & 0,0045 \\
\hline 1,2 & 3 & 650296 & 0,0016 & 1 & 319294 & 0,0016 & 25 & 103263135 & 0,0015 \\
\hline 1,3 & 3 & 650296 & $5,3279 \mathrm{e}-004$ & 1 & 319294 & $5,3278 \mathrm{e}-004$ & 17 & 70222455 & $5,1773 \mathrm{e}-004$ \\
\hline 1,4 & 3 & 650296 & $1,7922 \mathrm{e}-004$ & 1 & 319294 & $1,7922 \mathrm{e}-004$ & 15 & 61962285 & $1,7542 \mathrm{e}-004$ \\
\hline 1,5 & 3 & 650296 & $6,0443 \mathrm{e}-005$ & 1 & 319294 & $6,0443 \mathrm{e}-005$ & 13 & 53704113 & $5,9533 \mathrm{e}-005$ \\
\hline 1,6 & 3 & 650296 & $2,0431 \mathrm{e}-005$ & 1 & 319294 & $2,0431 \mathrm{e}-005$ & 11 & 45441945 & $2,0230 \mathrm{e}-005$ \\
\hline 1,7 & 3 & 650296 & $6,9219 \mathrm{e}-006$ & 1 & 319294 & $6,9219 \mathrm{e}-006$ & 7 & 28921605 & $6,8826 \mathrm{e}-006$ \\
\hline 1,8 & 3 & 650296 & $2,3502 \mathrm{e}-006$ & 1 & 319294 & $2,3502 \mathrm{e}-006$ & 11 & 45443943 & $2,3442 \mathrm{e}-006$ \\
\hline 1,9 & 3 & 650296 & $7,9977 \mathrm{e}-007$ & 1 & 319294 & $7,9977 \mathrm{e}-007$ & 6 & 24789522 & $7,9925 \mathrm{e}-007$ \\
\hline
\end{tabular}

Tabela 5.21: Resultados computacionais utilizando a função exp $x$.

\begin{tabular}{|l|c|c|r|r|r|r|}
\hline \multirow{2}{*}{ P } & \multicolumn{3}{|c|}{ PD } & \multicolumn{3}{c|}{ PDPC } \\
\cline { 2 - 7 } & It & Flops & \multicolumn{1}{c|}{ FO } & It & Flops & \multicolumn{1}{c|}{ FO } \\
\hline 1 & 19 & 4256095 & 0,0106 & 15 & 4285047 & 0,1194 \\
\hline 1,01 & 24 & 5367354 & 0,0111 & 20 & 5708020 & 0,0117 \\
\hline 1,1 & 28 & 6247570 & 0,0045 & 17 & 4864425 & 0,0045 \\
\hline 1,2 & 23 & 5147300 & 0,0015 & 18 & 5145623 & 0,0015 \\
\hline 1,3 & 21 & 4707192 & $5,1773 \mathrm{e}-004$ & 17 & 4864426 & $5,1773 \mathrm{e}-004$ \\
\hline 1,4 & 21 & 4707192 & $1,7542 \mathrm{e}-004$ & 18 & 5145623 & $1,7542 \mathrm{e}-004$ \\
\hline 1,5 & 19 & 4267084 & $5,9532 \mathrm{e}-005$ & 15 & 4302029 & $5,9533 \mathrm{e}-005$ \\
\hline 1,6 & 19 & 4267084 & $2,0228 \mathrm{e}-005$ & 15 & 4302030 & $2,0230 \mathrm{e}-005$ \\
\hline 1,7 & 18 & 4047030 & $6,8783 \mathrm{e}-006$ & 15 & 4302030 & $6,8824 \mathrm{e}-006$ \\
\hline 1,8 & 17 & 3826976 & $2,3385 \mathrm{e}-006$ & 14 & 4020832 & $2,3429 \mathrm{e}-006$ \\
\hline 1,9 & 17 & 3826976 & $7,9801 \mathrm{e}-007$ & 14 & 4020832 & $7,9870 \mathrm{e}-007$ \\
\hline
\end{tabular}

Tabela 5.22: Resultados computacionais utilizando a função exp $x$. 


\begin{tabular}{|l|r|r|r|r|r|r|r|r|c|}
\hline \multirow{2}{*}{ P } & \multicolumn{4}{|c|}{ BL } & \multicolumn{4}{c|}{ PC } & \multicolumn{3}{c|}{ GNCS } \\
\cline { 2 - 10 } & It & Flops & FO & It & Flops & FO & It & Flops & FO \\
\hline 1 & 6 & 2143591 & 0,0040 & 20 & 8303165 & 0,0017 & 31 & 132274711 & $2,4272 \mathrm{e}-004$ \\
\hline 1,01 & 9 & 3185356 & 0,0020 & 7 & 2972520 & 0,0023 & 31 & 132272713 & $2,0888 \mathrm{e}-004$ \\
\hline 1,1 & 4 & 1467396 & $5,4240 \mathrm{e}-005$ & 1 & 504384 & $5,4240 \mathrm{e}-005$ & 28 & 119473000 & $5,4133 \mathrm{e}-005$ \\
\hline 1,2 & 4 & 1467396 & $1,2118 \mathrm{e}-005$ & 1 & 504384 & $1,2118 \mathrm{e}-005$ & 25 & 106675285 & $1,2100 \mathrm{e}-005$ \\
\hline 1,3 & 4 & 1467396 & $2,7127 \mathrm{e}-006$ & 1 & 504384 & $2,7127 \mathrm{e}-006$ & 21 & 89607003 & $2,7099 \mathrm{e}-006$ \\
\hline 1,4 & 4 & 1467396 & $6,0848 \mathrm{e}-007$ & 1 & 504384 & $6,0848 \mathrm{e}-007$ & 15 & 64011573 & $6,0803 \mathrm{e}-007$ \\
\hline 1,5 & 4 & 1467396 & $1,3672 \mathrm{e}-007$ & 1 & 504384 & $1,3672 \mathrm{e}-007$ & 8 & 34143578 & $1,3666 \mathrm{e}-007$ \\
\hline 1,6 & 4 & 1467396 & $3,0772 \mathrm{e}-008$ & 1 & 504384 & $3,0772 \mathrm{e}-008$ & 6 & 25610436 & $3,0762 \mathrm{e}-008$ \\
\hline 1,7 & 4 & 1467396 & $6,9361 \mathrm{e}-009$ & 1 & 504384 & $6,9361 \mathrm{e}-009$ & 4 & 17077294 & $6,9349 \mathrm{e}-009$ \\
\hline 1,8 & 4 & 1467396 & $1,5656 \mathrm{e}-009$ & 1 & 504384 & $1,5656 \mathrm{e}-009$ & 3 & 12811722 & $1,5655 \mathrm{e}-009$ \\
\hline 1,9 & 4 & 1467396 & $3,5384 \mathrm{e}-010$ & 1 & 504384 & $3,5384 \mathrm{e}-010$ & 2 & 8543153 & $3,5384 \mathrm{e}-010$ \\
\hline
\end{tabular}

Tabela 5.23: Resultados computacionais utilizando a função exp $x$.

\begin{tabular}{|l|r|r|c|r|c|r|}
\hline \multirow{2}{*}{$\mathrm{P}$} & \multicolumn{4}{|c|}{$\mathrm{PD}$} & \multicolumn{3}{c|}{ PDPC } \\
\cline { 2 - 7 } & It & Flops & FO & It & Flops & FO \\
\hline 1 & 4 & 1581307 & $7,7105 \mathrm{e}-004$ & 3 & 1434009 & 0,0026 \\
\hline 1,01 & 31 & 11651632 & $1,6848 \mathrm{e}-004$ & 31 & 14060008 & $1,8536 \mathrm{e}-004$ \\
\hline 1,1 & 38 & 14259608 & $5,4133 \mathrm{e}-005$ & 33 & 14960652 & $4,4133 \mathrm{e}-005$ \\
\hline 1,2 & 36 & 13514472 & $1,2100 \mathrm{e}-005$ & 30 & 13609686 & $1,2100 \mathrm{e}-005$ \\
\hline 1,3 & 31 & 11651632 & $2,7099 \mathrm{e}-006$ & 25 & 11358075 & $2,7099 \mathrm{e}-006$ \\
\hline 1,4 & 21 & 7925952 & $6,0796 \mathrm{e}-007$ & 15 & 6854856 & $6,0803 \mathrm{e}-007$ \\
\hline 1,5 & 15 & 5690544 & $1,3655 \mathrm{e}-007$ & 16 & 7305178 & $1,3664 \mathrm{e}-007$ \\
\hline 1,6 & 14 & 5317976 & $3,0618 \mathrm{e}-008$ & 12 & 5503890 & $3,0721 \mathrm{e}-008$ \\
\hline 1,7 & 12 & 4572840 & $5,9809 \mathrm{e}-009$ & 11 & 5053568 & $6,8355 \mathrm{e}-009$ \\
\hline 1,8 & 12 & 4572840 & $1,3758 \mathrm{e}-009$ & 10 & 4603246 & $1,3471 \mathrm{e}-009$ \\
\hline 1,9 & 13 & 4945408 & $3,5384 \mathrm{e}-010$ & 10 & 4603246 & $3,0937 \mathrm{e}-010$ \\
\hline
\end{tabular}

Tabela 5.24: Resultados computacionais utilizando a função exp $x$. 


\begin{tabular}{|c|r|r|r|r|r|r|r|r|r|}
\hline \multirow{2}{*}{ P } & \multicolumn{4}{|c|}{ BL } & \multicolumn{3}{c|}{ PC } & \multicolumn{2}{c|}{ GNCS } \\
\cline { 2 - 10 } & It & Flops & FO & It & Flops & FO & It & \multicolumn{1}{c|}{ Flops } & FO \\
\hline 1 & 4 & 962567 & 0,0166 & 5 & 1456015 & 0,0165 & 42 & 174651816 & 0,0014 \\
\hline 1,01 & 5 & 1196718 & 0,0190 & 6 & 1751416 & 0,0162 & 34 & 141386520 & 0,0014 \\
\hline 1,1 & 5 & 1196718 & 0,0158 & 6 & 1751416 & 0,0168 & 26 & 108127218 & $3,7107 \mathrm{e}-004$ \\
\hline 1,2 & 4 & 973556 & $1,0570 \mathrm{e}-004$ & 1 & 359326 & $1,0570 \mathrm{e}-004$ & 22 & 91488576 & $1,0057 \mathrm{e}-004$ \\
\hline 1,3 & 4 & 973556 & $2,8378 \mathrm{e}-005$ & 1 & 359326 & $2,8378 \mathrm{e}-005$ & 14 & 58225278 & $2,7286 \mathrm{e}-005$ \\
\hline 1,4 & 4 & 973556 & $7,6374 \mathrm{e}-006$ & 1 & 359326 & $7,6374 \mathrm{e}-006$ & 15 & 62381442 & $7,4156 \mathrm{e}-006$ \\
\hline 1,5 & 4 & 973556 & $2,0613 \mathrm{e}-006$ & 1 & 359326 & $2,0613 \mathrm{e}-006$ & 10 & 41596626 & $2,0187 \mathrm{e}-006$ \\
\hline 1,6 & 4 & 973556 & $5,5783 \mathrm{e}-007$ & 1 & 359326 & $5,5783 \mathrm{e}-007$ & 7 & 29120142 & $5,5030 \mathrm{e}-007$ \\
\hline 1,7 & 4 & 973556 & $1,5137 \mathrm{e}-007$ & 1 & 359326 & $1,5137 \mathrm{e}-007$ & 9 & 37432470 & $1,5020 \mathrm{e}-007$ \\
\hline 1,8 & 4 & 973556 & $4,1185 \mathrm{e}-008$ & 1 & 359326 & $4,1185 \mathrm{e}-008$ & 5 & 20806815 & $4,1041 \mathrm{e}-008$ \\
\hline 1,9 & 4 & 973556 & $1,1236 \mathrm{e}-008$ & 1 & 359326 & $1,1236 \mathrm{e}-008$ & 4 & 16645656 & $1,1226 \mathrm{e}-008$ \\
\hline
\end{tabular}

Tabela 5.25: Resultados computacionais utilizando a função exp $x^{2}$.

\begin{tabular}{|l|r|r|r|r|c|r|}
\hline \multirow{2}{*}{$\mathrm{P}$} & \multicolumn{3}{|c|}{ PD } & \multicolumn{3}{c|}{ PDPC } \\
\cline { 2 - 7 } & It & Flops & \multicolumn{1}{c|}{ FO } & It & Flops & \multicolumn{1}{c|}{ FO } \\
\hline 1 & 12 & 3104571 & 0,0010 & 9 & 2927379 & 0,0083 \\
\hline 1,01 & 41 & 10427562 & 0,0011 & 19 & 6118202 & 0,0010 \\
\hline 1,1 & 40 & 10175424 & $3,7107 \mathrm{e}-004$ & 31 & 9926809 & $3,7107 \mathrm{e}-004$ \\
\hline 1,2 & 28 & 7149768 & $1,0057 \mathrm{e}-004$ & 24 & 7705121 & $1,0057 \mathrm{e}-004$ \\
\hline 1,3 & 22 & 5636940 & $2,7285 \mathrm{e}-005$ & 15 & 4848665 & $2,7286 \mathrm{e}-005$ \\
\hline 1,4 & 21 & 5384802 & $7,4155 \mathrm{e}-006$ & 20 & 6435585 & $7,4156 \mathrm{e}-006$ \\
\hline 1,5 & 18 & 4628388 & $2,0181 \mathrm{e}-006$ & 14 & 4531282 & $2,0187 \mathrm{e}-006$ \\
\hline 1,6 & 16 & 4124112 & $5,4902 \mathrm{e}-007$ & 12 & 3896514 & $5,4998 \mathrm{e}-007$ \\
\hline 1,7 & 15 & 3871974 & $1,4908 \mathrm{e}-007$ & 12 & 3896514 & $1,4995 \mathrm{e}-007$ \\
\hline 1,8 & 14 & 3619836 & $3,8956 \mathrm{e}-008$ & 11 & 3579130 & $4,0638 \mathrm{e}-008$ \\
\hline 1,9 & 16 & 4124112 & $9,6351 \mathrm{e}-009$ & 10 & 3261746 & $1,0377 \mathrm{e}-008$ \\
\hline
\end{tabular}

Tabela 5.26: Resultados computacionais utilizando a função exp $x^{2}$. 


\begin{tabular}{|c|r|r|r|r|r|r|r|r|c|}
\hline \multirow{2}{*}{ P } & \multicolumn{4}{|c|}{ BL } & \multicolumn{4}{c|}{ PC } & \multicolumn{3}{c|}{ GNCS } \\
\cline { 2 - 11 } & It & Flops & FO & It & Flops & FO & It & Flops & FO \\
\hline 1 & 7 & 3198452 & 0,0042 & 5 & 2685879 & 0,0053 & 24 & 104635770 & $2,4102 \mathrm{e}-004$ \\
\hline 1,01 & 16 & 7206044 & 0,0037 & 8 & 4263996 & 0,0027 & 21 & 91559675 & $2,0739 \mathrm{e}-004$ \\
\hline 1,1 & 4 & 1877240 & $5,3890 \mathrm{e}-005$ & 1 & 621350 & $5,3889 \mathrm{e}-005$ & 17 & 74124544 & $5,3704 \mathrm{e}-005$ \\
\hline 1,2 & 4 & 1877240 & $1,2024 \mathrm{e}-005$ & 1 & 621350 & $1,2024 \mathrm{e}-005$ & 18 & 78473586 & $1,1994 \mathrm{e}-005$ \\
\hline 1,3 & 4 & 1877240 & $2,6889 \mathrm{e}-006$ & 1 & 621350 & $2,6889 \mathrm{e}-006$ & 12 & 52321392 & $2,6840 \mathrm{e}-006$ \\
\hline 1,4 & 4 & 1877240 & $6,0249 \mathrm{e}-007$ & 1 & 621350 & $6,0249 \mathrm{e}-007$ & 9 & 39242298 & $6,0174 \mathrm{e}-007$ \\
\hline 1,5 & 4 & 1877240 & $1,3525 \mathrm{e}-007$ & 1 & 621350 & $1,3525 \mathrm{e}-007$ & 7 & 30522236 & $1,3514 \mathrm{e}-007$ \\
\hline 1,6 & 4 & 1877240 & $3,0412 \mathrm{e}-008$ & 1 & 621350 & $3,0412 \mathrm{e}-008$ & 7 & 30522236 & $3,0397 \mathrm{e}-008$ \\
\hline 1,7 & 4 & 1877240 & $6,8491 \mathrm{e}-009$ & 1 & 621350 & $6,8491 \mathrm{e}-009$ & 4 & 17445140 & $6,8474 \mathrm{e}-009$ \\
\hline 1,8 & 4 & 1877240 & $1,5448 \mathrm{e}-009$ & 1 & 621350 & $1,5448 \mathrm{e}-009$ & 2 & 8727076 & $1,5447 \mathrm{e}-009$ \\
\hline 1,9 & 4 & 1877240 & $3,4889 \mathrm{e}-010$ & 1 & 621350 & $3,4889 \mathrm{e}-010$ & 2 & 8727076 & $3,4889 \mathrm{e}-010$ \\
\hline
\end{tabular}

Tabela 5.27: Resultados computacionais utilizando a função exp $x^{2}$.

\begin{tabular}{|l|r|r|c|r|r|r|}
\hline \multirow{2}{*}{ P } & \multicolumn{4}{|c|}{ PD } & \multicolumn{3}{c|}{ PDPC } \\
\cline { 2 - 7 } & It & Flops & FO & It & Flops & FO \\
\hline 1 & 4 & 1991151 & $5,1699 \mathrm{e}-004$ & 3 & 1769019 & 0,0018 \\
\hline 1,01 & 37 & 17612559 & $1,9093 \mathrm{e}-004$ & 41 & 23041074 & $1,9699 \mathrm{e}-004$ \\
\hline 1,1 & 46 & 21869946 & $5,3704 \mathrm{e}-005$ & 34 & 19125666 & $5,3704 \mathrm{e}-005$ \\
\hline 1,2 & 40 & 19031688 & $1,1994 \mathrm{e}-005$ & 28 & 15769602 & $1,1994 \mathrm{e}-005$ \\
\hline 1,3 & 29 & 13828215 & $2,6840 \mathrm{e}-006$ & 25 & 14091570 & $2,6840 \mathrm{e}-006$ \\
\hline 1,4 & 22 & 10516914 & $6,0171 \mathrm{e}-007$ & 12 & 6820098 & $6,0157 \mathrm{e}-007$ \\
\hline 1,5 & 15 & 7205613 & $1,3502 \mathrm{e}-007$ & 18 & 10176162 & $1,3513 \mathrm{e}-007$ \\
\hline 1,6 & 13 & 6259527 & $3,0008 \mathrm{e}-008$ & 12 & 6820098 & $3,0363 \mathrm{e}-008$ \\
\hline 1,7 & 12 & 5786484 & $6,3107 \mathrm{e}-009$ & 12 & 6820098 & $6,7937 \mathrm{e}-009$ \\
\hline 1,8 & 11 & 5313441 & $1,5123 \mathrm{e}-009$ & 10 & 5701410 & $1,4143 \mathrm{e}-009$ \\
\hline 1,9 & 13 & 6259527 & $3,3540 \mathrm{e}-010$ & 8 & 4582722 & $2,8783 \mathrm{e}-010$ \\
\hline
\end{tabular}

Tabela 5.28: Resultados computacionais utilizando a função exp $x^{2}$. 


\begin{tabular}{|c|r|c|c|r|c|c|}
\hline \multirow{2}{*}{$\mathrm{P}$} & \multicolumn{3}{|c|}{ BL } & \multicolumn{3}{c|}{$\mathrm{PC}$} \\
\cline { 2 - 7 } & It & Tempo & Fo & It & Tempo & Fo \\
\hline 1,1 & 7 & $3,8589 \mathrm{e}+03$ & $3,3512 \mathrm{e}+04$ & 1 & $8,6112 \mathrm{e}+02$ & $3,3705 \mathrm{e}+04$ \\
\hline 1,2 & 8 & $8,1236 \mathrm{e}+03$ & $3,8426 \mathrm{e}+04$ & 10 & $9,8467 \mathrm{e}+03$ & $3,8426 \mathrm{e}+04$ \\
\hline 1,3 & 7 & $6,7028 \mathrm{e}+03$ & $4,4343 \mathrm{e}+04$ & 6 & $5,3258 \mathrm{e}+03$ & $4,4126 \mathrm{e}+04$ \\
\hline 1,4 & 7 & $7,1191 \mathrm{e}+03$ & $5,1428 \mathrm{e}+04$ & 6 & $6,2856 \mathrm{e}+03$ & $5,1938 \mathrm{e}+04$ \\
\hline 1,5 & 5 & $4,9047 \mathrm{e}+03$ & $5,9937 \mathrm{e}+04$ & 5 & $4,3646 \mathrm{e}+03$ & $5,9564 \mathrm{e}+04$ \\
\hline 1,6 & 6 & $6,5736 \mathrm{e}+03$ & $7,0179 \mathrm{e}+04$ & 5 & $5,3546 \mathrm{e}+03$ & $7,0165 \mathrm{e}+04$ \\
\hline 1,7 & 5 & $5,1326 \mathrm{e}+03$ & $8,2539 \mathrm{e}+04$ & 4 & $4,1257 \mathrm{e}+03$ & $8,3298 \mathrm{e}+04$ \\
\hline 1,8 & 5 & $4,7238 \mathrm{e}+03$ & $9,7482 \mathrm{e}+04$ & 5 & $4,9564 \mathrm{e}+03$ & $9,8147 \mathrm{e}+04$ \\
\hline 1,9 & 5 & $5,4242 \mathrm{e}+03$ & $1,1559 \mathrm{e}+05$ & 5 & $4,7919 \mathrm{e}+03$ & $1,1559 \mathrm{e}+05$ \\
\hline 2,0 & 4 & $3,9804 \mathrm{e}+03$ & $1,3757 \mathrm{e}+05$ & 4 & $4,9800 \mathrm{e}+03$ & $1,3757 \mathrm{e}+05$ \\
\hline
\end{tabular}

Tabela 5.29: Resultados computacionais utilizando o problema de grande porte.

\begin{tabular}{|c|r|r|c|r|c|c|}
\hline \multirow{2}{*}{$\mathrm{P}$} & \multicolumn{4}{|c|}{$\mathrm{PD}$} & \multicolumn{3}{c|}{ PDPC } \\
\cline { 2 - 7 } & It & Tempo & Fo & It & Tempo & Fo \\
\hline 1,1 & 1 & 741,1319 & $3,1918 \mathrm{e}+04$ & 1 & 887,9641 & $3,2480 \mathrm{e}+04$ \\
\hline 1,2 & 1 & 827,9342 & $3,6638 \mathrm{e}+04$ & 1 & 856,6936 & $3,6177 \mathrm{e}+04$ \\
\hline 1,3 & 1 & 801,2922 & $4,2435 \mathrm{e}+04$ & 1 & 826,9323 & $4,0170 \mathrm{e}+04$ \\
\hline 1,4 & 1 & 831,6956 & $4,9537 \mathrm{e}+04$ & 1 & 832,6895 & $4,5249 \mathrm{e}+04$ \\
\hline 1,5 & 1 & 843,3268 & $5,8176 \mathrm{e}+04$ & 5 & $4,2766 \mathrm{e}+03$ & $5,9846 \mathrm{e}+04$ \\
\hline 1,6 & 7 & $5,5551 \mathrm{e}+03$ & $6,8518 \mathrm{e}+04$ & 5 & $4,1477 \mathrm{e}+03$ & $7,0128 \mathrm{e}+04$ \\
\hline 1,7 & 8 & $6,9422 \mathrm{e}+03$ & $8,1549 \mathrm{e}+04$ & 5 & $7,1247 \mathrm{e}+03$ & $8,2465 \mathrm{e}+04$ \\
\hline 1,8 & 8 & $7,1460 \mathrm{e}+03$ & $9,6172 \mathrm{e}+04$ & 5 & $4,5319 \mathrm{e}+03$ & $9,7388 \mathrm{e}+04$ \\
\hline 1,9 & 8 & $6,9720 \mathrm{e}+03$ & $1,1392 \mathrm{e}+05$ & 5 & $4,7974 \mathrm{e}+03$ & $1,1635 \mathrm{e}+05$ \\
\hline 2,0 & 9 & $7,8416 \mathrm{e}+03$ & $1,3737 \mathrm{e}+05$ & 6 & $5,4329 \mathrm{e}+03$ & $1,3615 \mathrm{e}+05$ \\
\hline
\end{tabular}

Tabela 5.30: Resultados computacionais utilizando o problema de grande porte. 


\begin{tabular}{|c|r|c|c|}
\hline \multirow{2}{*}{$\mathrm{P}$} & \multicolumn{3}{|c|}{ GNCS } \\
\cline { 2 - 4 } & It & Tempo & Fo \\
\hline 1,1 & 3 & $5,8997 \mathrm{e}+03$ & $3,3542 \mathrm{e}+04$ \\
\hline 1,2 & 3 & $5,8686 \mathrm{e}+03$ & $3,8475 \mathrm{e}+04$ \\
\hline 1,3 & 3 & $5,8200 \mathrm{e}+03$ & $4,4374 \mathrm{e}+04$ \\
\hline 1,4 & 5 & $9,7496 \mathrm{e}+03$ & $5,1434 \mathrm{e}+04$ \\
\hline 1,5 & 5 & $1,0070 \mathrm{e}+04$ & $5,9937 \mathrm{e}+04$ \\
\hline 1,6 & 5 & $1,2327 \mathrm{e}+04$ & $7,0179 \mathrm{e}+04$ \\
\hline 1,7 & 5 & $1,0039 \mathrm{e}+04$ & $8,2539 \mathrm{e}+04$ \\
\hline 1,8 & 4 & $7,5788 \mathrm{e}+03$ & $9,7483 \mathrm{e}+04$ \\
\hline 1,9 & 4 & $7,6355 \mathrm{e}+03$ & $1,1559 \mathrm{e}+05$ \\
\hline 2,0 & 2 & $3,9044 \mathrm{e}+03$ & $1,3757 \mathrm{e}+05$ \\
\hline
\end{tabular}

Tabela 5.31: Resultados computacionais utilizando o problema de grande porte. 


\section{Capítulo 6}

\section{Conclusões e Perspectivas Futuras}

\subsection{Conclusões}

Os métodos de pontos interiores aplicados ao problema de regressão pela norma $L_{p}$ apresentam resultados computacionais superiores ao método GNCS em relação à robustez, iteração e número de flops, repetindo o desempenho obtido na minimização pelas normas $L_{1}$ e $L_{\infty}$ em [20, 21], respectivamente.

Podemos verificar também que os métodos de pontos interiores apresentam desempenho computacional superior ao método GNCS em relação à validade da solução e ao tempo computacional.

Para o método GNCS o critério de convergência (3.23) proposto em [13] não converge para problemas de grande porte e, em outros casos, não consegue uma aproximação da função objetivo e resulta um número muito grande de iterações.

Os métodos de pontos interiores convergem para problemas de grande porte com função objetivo e esforço por iteração menores que o método GNCS.

$\mathrm{Na}$ maioria dos casos, o método preditor-corretor apresenta desempenho computacional superior ao método barreira logarítmica assim como o método primal-dual preditor- 
corretor em relação ao método primal-dual barreira logarítmica.

Em relação à função objetivo, os métodos de pontos interiores barreira logarítmica e preditor-corretor apresentam em geral desempenho computacional ligeiramente inferior aos métodos primal-dual barreira logarítmica e primal-dual preditor-corretor respectivamente.

Utilizando $p=1$ podemos verificar que os métodos de pontos interiores não obtiveram bons resultados, pois a derivada da norma 1 não é bem definida. Neste caso, o problema pode ser modelado como um problema de otimização linear e portanto este método não seria utilizado.

\subsection{Perspectivas Futuras}

Implementação em C/FORTRAN, explorando a estrutura matricial (4.1.3). Explorar a estrutura matricial dessa forma em Matlab não compensa.

Realizar experimentos utilizando novas idéias como, por exemplo, utilizar o gap como critério de parada. 


\section{Bibliografia}

[1] I. Adler, M.G.C. Resende, G. Veiga and N. Karmarkar, An implementation of Karmarkar's algorithm for linear programming, Mathematical Programming, 44 (1989), 297-335.

[2] M.S. Bazaraa, "Linear Programming and Network Flows", 2nd ed., John Wiley \& Sons, 1990.

[3] M.S. Bazaraa, H.D. Sherali and C.M. Shetty, "Nonlinear Programming: Theory and Algorithms", John Wiley \& Sons, 1979.

[4] D.P. Bertsekas, "Nonlinear Programming", 2nd ed., Athena Scientific, USA, 1999.

[5] J. Castro, A specialized interior-point algorithm for multcommodity network flows, SIAM J. Optimization, 10 (2000), 852-877.

[6] T.F. Coleman and Y. Li, A globally and quadratically convergent affine scaling method for linear $l_{1}$ problems, Math. Programming, 56 (1992), 189-222.

[7] A.S. El-Bakry, R.A. Tapia, T. Tsuchiya and Y. Zhang, On the formulation and the theory of the Newton interior-point method for nonlinear programming, Journal of Optimization Theory and Applications, 89 (1996), 507-541.

[8] A. Dax and B. Berkowitz, Column relaxation methods for least norm problems, SIAM J. Sci. Stat. Comput., 11 (1990), 975-989.

[9] G.H. Golub and C.F. Van Loan, "Matrix Computations", 3rd ed., The Johns Hopkins University Press, Baltimore, MA, 1996. 
[10] J. Gondzio, Multiple centrality corrections in a primal-dual method for linear programming, Computational Optimization and Applications, 6 (1996), 137-156.

[11] G. Heinig and P. Jankowski, Parallel and superfast algorithms for Hankel systems, Numerical Mathematics, 58 (1990), 109-127.

[12] J.L. Kennington and R.V. Helgason, "Algorithms for Network Programming", Wiley, New York, 1980.

[13] Y. Li, A globally convergent method for $l_{p}$ problems, SIAM J. Optimization, 3 (1993), 609-629.

[14] D.G. Luenberger, "Linear and Nonlinear Programming", Second Edition, AddisonWesley, 1989.

[15] I.J. Lustig, R.E. Marsten and D.F. Shanno, On implementing Mehrotra's predictorcorrector interior point method for linear programming, SIAM J. Optimization, 2 (1992), 435-449.

[16] S. Mehrotra, On the implementation of a primal-dual interior point method, SIAM Journal on Optimization, 2 (1992), 575-601.

[17] G. Merle and H. Späcth, Computational experience with discrete $l_{p}$ approximation, Computing, 12 (1974), 315-321.

[18] R.D.C. Monteiro, I. Adler and M.G.C. Resende, A polynomial-time primal-dual affine scaling algorithm for linear and convex quadratic programming and its power series extension, Mathematics of Operations Research, 15 (1990), 191-214.

[19] A.R.L. Oliveira and C. Lyra, Implementação de um método de pontos interiores para programação linear, Revista SBA: Controle $\mathscr{E}$ Automação, 3 (1991), 370-382.

[20] A.R.L. Oliveira and C. Lyra, Interior point methods for the polynomial $L_{\infty}$ fitting problems, Internacional Transactions in Operational Research, 11 (3) (2004), 309-322. 
[21] A.R.L. Oliveira, M.A. Nascimento and C. Lyra, Efficient implementation and benchmark of interior point methods for the polynomial $L_{1}$ fitting problems, Statistics 83 Data Analysis, 35 (2000), 119-135.

[22] A.R.L. Oliveira, L. Nepomuceno and S. Soares, Optimal active power dispatch combining network flow and interior point approaches, IEEE Transactions on Power Systems, 18 (4) (2003), 1235-1240.

[23] A.R.L. Oliveira, L. Nepomuceno and S. Soares, Short term hydroelectric sheduling combining network flow and interior point approaches, Trabalho aceito para publicação na Electrical Power 8 Energy Systems, (2004).

[24] A.R.L. Oliveira and S. Soares, Métodos de pontos interiores para problema de fluxo de potência ótimo DC, Controle $\mathscr{G}$ Automação, 14 (3) (2003), 278-285.

[25] M.G.C. Resende and G. Veiga, An efficient implementation of a network interior point method, DIMACS Series in Discrete Mathematics and Theoretical Computer Science, 12 (1993), 299-348.

[26] R. Sedgewick, "Algorithms", Addison-Wesley, Reading, MA, 1983.

[27] R.A. Tapia and Yin Zhang, Superlinear and quadratic convergence of primal-dual interior point methods for linear programming revisited, Journal of Optimization Theory and Applications, 73 (1992), 229-242.

[28] W.F. Trench, An algorithm for the inversion of finite Hankel matrices, J. Soc. Ind. Appl. Math., 13 (4) (1965), 1102-1107.

[29] R.J. Vanderbei, "Linear Programming - Foundations and Extensions", Kluwer Academics Publishers, Boston, USA, 1996.

[30] S.J. Wright, "Primal-Dual Interior-Point Methods", SIAM Publications, SIAM Philadelphia, PA, USA, 1996. 